\title{
Möbius and Hückel Cyclacenes with Dewar and Ladenburg Defects
}

Magnus W. D. Hanson-Heine* and Jonathan D. Hirst

School of Chemistry, University of Nottingham, University Park, Nottingham NG7 2RD.

*magnus.hansonheine@nottingham.ac.uk 
Table S1. Calculated TAO-DFT singlet $\left(\mathrm{S}_{0}\right)$ state energies in $\mathrm{E}_{\mathrm{h}}$.

\begin{tabular}{ccc}
\hline$n$-cyclacene & $\begin{array}{c}\text { Isomers Containing } \\
\text { One Ladenburg } \\
\text { Benzenoid }\end{array}$ & $\begin{array}{c}\text { Isomers Containing } \\
\text { Two Ladenburg } \\
\text { Benzenoids }\end{array}$ \\
\hline Hückel-[5]cyclacene & -767.015147 & -767.004109 \\
Hückel-[6]cyclacene & -920.542730 & -920.549562 \\
Hückel-[7]cyclacene & -1074.063793 & -1074.043546 \\
Hückel-[8]cyclacene & -1227.569604 & -1227.558188 \\
Hückel-[9]cyclacene & -1381.076439 & -1381.045231 \\
Hückel-[10]cyclacene & -1534.574878 & -1534.544430 \\
Hückel-[11]cyclacene & -1688.073202 & -1688.028263 \\
Hückel-[12]cyclacene & -1841.567984 & -1841.520198 \\
Hückel-[13]cyclacene & -1995.061832 & -1995.003803 \\
Hückel-[14]cyclacene & -2148.557500 & -2148.492210 \\
\hline
\end{tabular}

Table S2. Calculated TAO-DFT triplet $\left(\mathrm{T}_{1}\right)$ state energies in $\mathrm{E}_{\mathrm{h}}$.

\begin{tabular}{ccc}
\hline$n$-cyclacene & $\begin{array}{c}\text { Isomers Containing } \\
\text { One Ladenburg } \\
\text { Benzenoid }\end{array}$ & $\begin{array}{c}\text { Isomers Containing } \\
\text { Two Ladenburg } \\
\text { Benzenoids }\end{array}$ \\
\hline Hückel-[5]cyclacene & -766.993315 & -766.936114 \\
Hückel-[6]cyclacene & -920.511277 & -920.472849 \\
Hückel-[7]cyclacene & -1074.033595 & -1073.971154 \\
Hückel-[8]cyclacene & -1227.548123 & -1227.486095 \\
Hückel-[9]cyclacene & -1381.058884 & -1380.984195 \\
Hückel-[10]cyclacene & -1534.562731 & -1534.489130 \\
Hückel-[11]cyclacene & -1688.062832 & -1687.984750 \\
Hückel-[12]cyclacene & -1841.559609 & -1841.483802 \\
Hückel-[13]cyclacene & -1995.054395 & -1994.975667 \\
Hückel-[14]cyclacene & -2148.551032 & -2148.469834 \\
\hline
\end{tabular}

Table S3. Calculated TAO-DFT singlet $\left(\mathrm{S}_{0}\right)$ state energies in $\mathrm{E}_{\mathrm{h}}$.

\begin{tabular}{ccc}
\hline$n$-cyclacene & $\begin{array}{c}\text { Isomers Containing No } \\
\text { Dewar Benzenoids }\end{array}$ & $\begin{array}{c}\text { Isomers Containing } \\
\text { One Dewar Benzenoid }\end{array}$ \\
\hline Möbius-[9]cyclacene & -1380.930611 & -1380.933157 \\
Möbius-[10]cyclacene & -1534.460533 & -1534.457964 \\
Möbius-[11]cyclacene & -1687.983389 & -1687.975612 \\
Möbius-[12]cyclacene & -1841.498131 & -1841.482095 \\
Möbius-[13]cyclacene & -1995.009443 & -1994.990498 \\
Möbius-[14]cyclacene & -2148.516723 & -2148.490708 \\
\hline
\end{tabular}


Table S4. Calculated TAO-DFT triplet $\left(\mathrm{T}_{1}\right)$ state energies in $\mathrm{E}_{\mathrm{h}}$.

\begin{tabular}{ccc}
\hline$n$-cyclacene & $\begin{array}{c}\text { Isomers Containing No } \\
\text { Dewar Benzenoids }\end{array}$ & $\begin{array}{c}\text { Isomers Containing } \\
\text { One Dewar Benzenoid }\end{array}$ \\
\hline Möbius-[9]cyclacene & -1380.925193 & -1380.926856 \\
Möbius-[10]cyclacene & -1534.454410 & -1534.449638 \\
Möbius-[11]cyclacene & -1687.977405 & -1687.967539 \\
Möbius-[12]cyclacene & -1841.493113 & -1841.476188 \\
Möbius-[13]cyclacene & -1995.004528 & -1994.983655 \\
Möbius-[14]cyclacene & -2148.512482 & -2148.485758 \\
\hline
\end{tabular}


Table S5. Cartesian Coordinates in Angstrom for the optimized structure of Hückel-[5]cyclacene containing one Ladenburg benzenoid.

\begin{tabular}{cccc}
\hline Atom & x-coordinate & y-coordinate & z-coordinate \\
\hline C & 2.2896311783 & -1.3985110354 & -0.0000029084 \\
C & 2.2896310683 & 1.3985111656 & -0.0000179153 \\
H & 2.2377153332 & 2.4852577138 & -0.0000238306 \\
H & 2.2377157201 & -2.4852575997 & 0.0000036644 \\
C & 1.8177953407 & -0.7164462494 & -1.1602119530 \\
C & 1.8177951820 & 0.7164334239 & -1.1602192723 \\
C & 1.8178072610 & -0.7164329145 & 1.1602031049 \\
C & 1.8178072830 & 0.7164459549 & 1.1601957393 \\
C & 0.6360596303 & 1.3993664532 & -1.6466231609 \\
C & 0.6360600046 & -1.3993843876 & -1.6466068143 \\
C & 0.6360756423 & 1.3993852719 & 1.6466025326 \\
$\mathrm{C}$ & 0.6360751926 & -1.3993674959 & 1.6466177399 \\
$\mathrm{C}$ & -1.7048658026 & -1.4685864771 & -0.8408063906 \\
$\mathrm{C}$ & -1.7048659850 & 1.4685768594 & -0.8408245699 \\
$\mathrm{C}$ & -0.5620421307 & -0.7228880250 & -1.4979543921 \\
$\mathrm{C}$ & -0.5620425903 & 0.7228717804 & -1.4979635743 \\
$\mathrm{H}$ & 0.6272105707 & 2.4867490724 & -1.6076525370 \\
$\mathrm{H}$ & 0.6272112051 & -2.4867665320 & -1.6076232250 \\
$\mathrm{H}$ & 0.6272275978 & 2.4867675067 & 1.6076206411 \\
$\mathrm{H}$ & 0.6272269680 & -2.4867501614 & 1.6076477968 \\
$\mathrm{C}$ & -0.5620274360 & -0.7228727360 & 1.4979677924 \\
$\mathrm{C}$ & -2.5854608955 & 0.6738567175 & 0.0000086434 \\
$\mathrm{C}$ & -0.5620269898 & 0.7228887405 & 1.4979595222 \\
$\mathrm{C}$ & -1.7048578156 & -1.4685766524 & 0.8408406358 \\
$\mathrm{C}$ & -2.5854617296 & -0.6738563325 & 0.0000165228 \\
$\mathrm{C}$ & -1.7048574886 & 1.4685860184 & 0.8408240457 \\
$\mathrm{H}$ & -1.9804235229 & -2.4453815444 & 1.2360801562 \\
$\mathrm{H}$ & -1.9804214494 & 2.4453960399 & 1.2360520819 \\
$\mathrm{H}$ & -1.9804354583 & 2.4453822429 & -1.2360601906 \\
$\mathrm{H}$ & -1.9804349683 & -2.4453956093 & -1.2360331259 \\
\hline & & &
\end{tabular}


Table S6. Cartesian Coordinates in Angstrom for the optimized structure of Hückel-[5]cyclacene containing two Ladenburg benzenoids.

\begin{tabular}{cccc}
\hline Atom & x-coordinate & y-coordinate & z-coordinate \\
\hline $\mathrm{H}$ & -2.4660089638 & -2.4388636411 & -0.6272758889 \\
$\mathrm{C}$ & -2.0321489262 & -1.4769037016 & -0.3611688615 \\
$\mathrm{C}$ & -2.0321513760 & 1.4769028877 & -0.3611641413 \\
$\mathrm{C}$ & -1.2044390254 & -0.7018736613 & -1.3717480899 \\
$\mathrm{C}$ & -1.2044401577 & 0.7018776779 & -1.3717458665 \\
$\mathrm{H}$ & -2.4660134821 & 2.4388627456 & -0.6272679826 \\
$\mathrm{C}$ & -0.0000018579 & -1.3729232967 & -1.6449426285 \\
$\mathrm{C}$ & -0.0000036384 & 1.3729300904 & -1.6449365400 \\
$\mathrm{H}$ & -0.0000044805 & 2.4598241130 & -1.5803298394 \\
$\mathrm{H}$ & -0.0000010940 & -2.4598175925 & -1.5803407580 \\
$\mathrm{C}$ & 0.0000024322 & -0.6834677711 & 1.2619503634 \\
$\mathrm{C}$ & 0.0000018976 & 0.6834640565 & 1.2619516767 \\
$\mathrm{C}$ & -2.4705597937 & 0.6674610677 & 0.7642775331 \\
$\mathrm{C}$ & -2.4705601766 & -0.6674669584 & 0.7642757307 \\
$\mathrm{C}$ & -1.2997811947 & 1.4605322903 & 1.1231794429 \\
$\mathrm{C}$ & -1.2997798837 & -1.4605371496 & 1.1231752389 \\
$\mathrm{H}$ & -1.3722193971 & -2.4175775579 & 1.6392257943 \\
$\mathrm{H}$ & -1.3722221727 & 2.4175704685 & 1.6392339364 \\
$\mathrm{H}$ & 1.3722249307 & -2.4175783638 & 1.6392187375 \\
$\mathrm{C}$ & 1.2997839364 & -1.4605362193 & 1.1231717912 \\
$\mathrm{C}$ & 1.2997846350 & 1.4605333991 & 1.1231750818 \\
$\mathrm{H}$ & 1.3722279555 & 2.4175696476 & 1.6392329383 \\
$\mathrm{C}$ & 1.2044351544 & -0.7018721453 & -1.3717520303 \\
$\mathrm{C}$ & 1.2044339499 & 0.7018795091 & -1.3717483094 \\
$\mathrm{C}$ & 2.4705623463 & 0.6674612400 & 0.7642700338 \\
$\mathrm{C}$ & 2.4705632394 & -0.6674667545 & 0.7642677338 \\
$\mathrm{C}$ & 2.0321478898 & 1.4769049920 & -0.3611682443 \\
$\mathrm{C}$ & 2.0321494282 & -1.4769015916 & -0.3611769957 \\
$\mathrm{H}$ & 2.4660062233 & -2.4388628505 & -0.6272843605 \\
$\mathrm{H}$ & 2.4660114030 & 2.4388634952 & -0.6272750195 \\
\hline & & &
\end{tabular}


Table S7. Cartesian Coordinates in Angstrom for the optimized structure of Hückel-[6]cyclacene containing one Ladenburg benzenoid.

\begin{tabular}{cccc}
\hline Atom & x-coordinate & y-coordinate & z-coordinate \\
\hline H & -2.2498536725 & 1.1352444009 & 2.4924752700 \\
C & -2.2971258932 & 1.1693844566 & 1.4062775760 \\
C & -2.2971262744 & 1.1693765557 & -1.4062837965 \\
C & -2.7559142902 & -0.0000011650 & 0.7196109792 \\
C & -2.7559145051 & -0.0000052293 & -0.7196104523 \\
H & -2.2498543013 & 1.1352303205 & -2.4924813152 \\
C & -2.2971228275 & -1.1693817651 & 1.4062841832 \\
$\mathrm{C}$ & -2.2971231983 & -1.1693896498 & -1.4062771538 \\
H & -2.2498511438 & -1.1352493095 & -2.4924748378 \\
H & -2.2498504466 & -1.1352353136 & 2.4924816672 \\
$\mathrm{C}$ & -1.2824540963 & 1.8574972893 & 0.7222952941 \\
$\mathrm{C}$ & -1.2824542017 & 1.8574930994 & -0.7223056231 \\
$\mathrm{C}$ & -1.2824493562 & -1.8574959001 & 0.7223057652 \\
$\mathrm{C}$ & -1.2824495741 & -1.8574999767 & -0.7222951705 \\
$\mathrm{C}$ & -0.0105966198 & 1.8994693595 & -1.4034867375 \\
$\mathrm{C}$ & -0.0105963806 & 1.8994774986 & 1.4034759543 \\
$\mathrm{C}$ & -0.0105919073 & -1.8994772015 & -1.4034760830 \\
$\mathrm{C}$ & -0.0105915940 & -1.8994695634 & 1.4034866162 \\
$\mathrm{C}$ & 2.2357774352 & 0.8366063786 & 1.4733033707 \\
$\mathrm{C}$ & 2.2357773298 & 0.8365979277 & -1.4733082961 \\
$\mathrm{C}$ & 1.1311967758 & 1.5369658307 & 0.7281717516 \\
$\mathrm{C}$ & 1.1311966672 & 1.5369616003 & -0.7281806540 \\
$\mathrm{H}$ & -0.0146269841 & 1.8609483622 & -2.4912482173 \\
$\mathrm{H}$ & -0.0146264294 & 1.8609627629 & 2.4912376520 \\
$\mathrm{H}$ & -0.0146222841 & -1.8609620873 & -2.4912377725 \\
$\mathrm{H}$ & -0.0146217072 & -1.8609485914 & 2.4912480773 \\
$\mathrm{C}$ & 1.1312007948 & -1.5369592387 & 0.7281803395 \\
$\mathrm{C}$ & 3.1146069289 & 0.0000018718 & -0.6687472323 \\
$\mathrm{C}$ & 1.1312005689 & -1.5369632230 & -0.7281720367 \\
$\mathrm{C}$ & 2.2357795721 & -0.8365929837 & 1.4733080476 \\
$\mathrm{C}$ & 3.1146073385 & 0.0000056122 & 0.6687475346 \\
$\mathrm{C}$ & 2.2357795468 & -0.8366008740 & -1.4733036640 \\
$\mathrm{H}$ & 2.5275645246 & -1.2333640496 & 2.4450064377 \\
$\mathrm{H}$ & 2.5275649794 & -1.2333779801 & -2.4449994481 \\
$\mathrm{H}$ & 2.5275620396 & 1.2333703617 & -2.4450062000 \\
$\mathrm{H}$ & 2.5275612335 & 1.2333837177 & 2.4449995500 \\
\hline & & & \\
& & & \\
& &
\end{tabular}


Table S8. Cartesian Coordinates in Angstrom for the optimized structure of Hückel-[6]cyclacene containing two Ladenburg benzenoids.

\begin{tabular}{cccc}
\hline Atom & x-coordinate & y-coordinate & z-coordinate \\
\hline H & -1.2559453123 & -2.4309549491 & 2.6622929593 \\
C & -0.8242972561 & -1.4722210384 & 2.3795021231 \\
C & -0.8243379532 & 1.4721979808 & 2.3795020946 \\
H & -1.2560123156 & 2.4309200495 & 2.6622930524 \\
C & 1.4319225388 & -0.7065209142 & 1.2249535913 \\
C & 1.4319029291 & 0.7065609675 & 1.2249536118 \\
C & -0.0000092314 & 0.6669724332 & 3.2711961636 \\
$\mathrm{C}$ & 0.0000092314 & -0.6669724332 & 3.2711961636 \\
$\mathrm{C}$ & 0.8242972561 & 1.4722210384 & 2.3795021231 \\
$\mathrm{C}$ & 0.8243379532 & -1.4721979808 & 2.3795020946 \\
$\mathrm{H}$ & 1.2560123156 & -2.4309200495 & 2.6622930524 \\
$\mathrm{H}$ & 1.2559453123 & 2.4309549491 & 2.6622929593 \\
$\mathrm{H}$ & 1.2560123156 & -2.4309200495 & -2.6622930524 \\
$\mathrm{C}$ & 0.8243379532 & -1.4721979808 & -2.3795020946 \\
$\mathrm{C}$ & 0.8242972561 & 1.4722210384 & -2.3795021231 \\
$\mathrm{C}$ & 1.4319225388 & -0.7065209142 & -1.2249535913 \\
$\mathrm{C}$ & 1.4319029291 & 0.7065609675 & -1.2249536118 \\
$\mathrm{H}$ & 1.2559453123 & 2.4309549491 & -2.6622929593 \\
$\mathrm{C}$ & 1.5566748350 & -1.3766272969 & 0.0000000000 \\
$\mathrm{C}$ & 1.5566368091 & 1.3766709305 & 0.0000000000 \\
$\mathrm{H}$ & 1.4945434516 & 2.4638888029 & 0.0000000000 \\
$\mathrm{H}$ & 1.4946122023 & -2.4638469522 & 0.0000000000 \\
$\mathrm{C}$ & -1.4319029291 & -0.7065609675 & 1.2249536118 \\
$\mathrm{C}$ & -1.4319225388 & 0.7065209142 & 1.2249535913 \\
$\mathrm{C}$ & -1.5566368091 & -1.3766709305 & 0.0000000000 \\
$\mathrm{C}$ & -1.5566748350 & 1.3766272969 & 0.0000000000 \\
$\mathrm{H}$ & -1.4946122023 & 2.4638469522 & 0.0000000000 \\
$\mathrm{H}$ & -1.4945434516 & -2.463888029 & 0.0000000000 \\
$\mathrm{C}$ & -1.4319029291 & -0.7065609675 & -1.2249536118 \\
$\mathrm{C}$ & -1.4319225388 & 0.7065209142 & -1.2249535913 \\
$\mathrm{C}$ & -0.0000092314 & 0.6669724332 & -3.2711961636 \\
$\mathrm{C}$ & 0.0000092314 & -0.6669724332 & -3.2711961636 \\
$\mathrm{C}$ & -0.8243379532 & 1.4721979808 & -2.3795020946 \\
$\mathrm{C}$ & -0.8242972561 & -1.4722210384 & -2.3795021231 \\
$\mathrm{H}$ & -1.2559453123 & -2.4309549491 & -2.6622929593 \\
$\mathrm{H}$ & -1.2560123156 & 2.4309200495 & -2.6622930524 \\
\hline & & & \\
\hline
\end{tabular}


Table S9. Cartesian Coordinates in Angstrom for the optimized structure of Hückel-[7]cyclacene containing one Ladenburg benzenoid.

\begin{tabular}{cccc}
\hline Atom & x-coordinate & y-coordinate & z-coordinate \\
\hline H & -3.1026251949 & 0.0000292636 & 2.4955889164 \\
C & -3.1582550645 & 0.0000301850 & 1.4094052626 \\
C & -3.1582551957 & 0.0000300580 & -1.4094054390 \\
C & -2.8016318771 & -1.1860905984 & 0.7250868600 \\
C & -2.8016317902 & -1.1860905592 & -0.7250869655 \\
H & -3.1026249481 & 0.0000289434 & -2.4955890698 \\
H & -1.8203395383 & 1.9506658609 & 2.4966514822 \\
$\mathrm{C}$ & -1.8465465557 & 1.9965932749 & 1.4101036132 \\
$\mathrm{C}$ & -1.8465465777 & 1.9965930515 & -1.4101040493 \\
$\mathrm{C}$ & -2.8016091098 & 1.1861431705 & 0.7250871385 \\
$\mathrm{C}$ & -2.8016090925 & 1.1861430469 & -0.7250875486 \\
$\mathrm{H}$ & -1.8203393757 & 1.9506655749 & -2.4966518945 \\
$\mathrm{C}$ & -1.8465834897 & -1.9965572300 & 1.4101046014 \\
$\mathrm{C}$ & -1.8465834167 & -1.9965576018 & -1.4101043497 \\
$\mathrm{H}$ & -1.8203759111 & -1.9506317132 & -2.4966523055 \\
$\mathrm{H}$ & -1.8203763151 & -1.9506310832 & 2.4966525491 \\
$\mathrm{C}$ & -0.6618911390 & 2.2741082038 & 0.7253669251 \\
$\mathrm{C}$ & -0.6618911507 & 2.2741080331 & -0.7253671866 \\
$\mathrm{C}$ & -0.6619336422 & -2.2740966724 & 0.7253673451 \\
$\mathrm{C}$ & -0.6619336390 & -2.2740968009 & -0.7253667517 \\
$\mathrm{C}$ & 0.5919449784 & 2.0690479503 & -1.4036465535 \\
$\mathrm{C}$ & 0.5919448965 & 2.0690482191 & 1.4036463790 \\
$\mathrm{C}$ & 0.5919064890 & -2.0690609955 & -1.4036459659 \\
$\mathrm{C}$ & 0.5919063311 & -2.0690605772 & 1.4036465995 \\
$\mathrm{C}$ & 2.7575004032 & 0.8323153803 & 1.4628794077 \\
$\mathrm{C}$ & 2.7575000131 & 0.8323149619 & -1.4628795612 \\
$\mathrm{C}$ & 1.6730244291 & 1.5593715072 & 0.7295085912 \\
$\mathrm{C}$ & 1.6730245947 & 1.5593713979 & -0.7295085711 \\
$\mathrm{H}$ & 0.5884414375 & 2.0569510597 & -2.4919587543 \\
$\mathrm{H}$ & 0.5884411989 & 2.0569516325 & 2.4919585752 \\
$\mathrm{H}$ & 0.5884023257 & -2.0569623181 & -2.4919581730 \\
$\mathrm{H}$ & 0.5884020600 & -2.0569617027 & 2.4919588012 \\
$\mathrm{C}$ & 1.6729945796 & -1.5594022929 & 0.7295091813 \\
$\mathrm{C}$ & 3.6526162546 & -0.0000342563 & -0.6711974824 \\
$\mathrm{C}$ & 1.6729946923 & -1.5594025423 & -0.7295085400 \\
$\mathrm{C}$ & 2.7574826542 & -0.8323650955 & 1.4628794512 \\
$\mathrm{C}$ & 3.6526152424 & -0.0000341002 & 0.6711966377 \\
$\mathrm{C}$ & 2.7574824894 & -0.8323653209 & -1.4628792548 \\
$\mathrm{H}$ & 3.0261850451 & -1.2080377484 & 2.4497450412 \\
$\mathrm{H}$ & 3.0261836964 & -1.2080382114 & -2.4497451016 \\
$\mathrm{H}$ & 3.0262088854 & 1.2079833529 & -2.4497450231 \\
$\mathrm{H}$ & 3.0262101950 & 1.2079842379 & 2.4497444315 \\
\hline & & &
\end{tabular}


Table S10. Cartesian Coordinates in Angstrom for the optimized structure of Hückel-[7]cyclacene containing two Ladenburg benzenoids.

\begin{tabular}{|c|c|c|c|}
\hline Atom & $\mathrm{x}$-coordinate & $\mathrm{y}$-coordinate & z-coordinate \\
\hline $\mathrm{H}$ & -2.4380382847 & -0.5507097174 & -3.5184910183 \\
\hline $\mathrm{C}$ & -1.4729716127 & -0.2604075300 & -3.1058898354 \\
\hline $\mathrm{C}$ & 1.4729638632 & -0.2604250719 & -3.1058961162 \\
\hline $\mathrm{C}$ & -0.7171064288 & -1.2552942673 & -2.2637265631 \\
\hline $\mathrm{C}$ & 0.7170918527 & -1.2553014721 & -2.2637256256 \\
\hline $\mathrm{H}$ & 2.4380182410 & -0.5507445867 & -3.5185141315 \\
\hline $\mathrm{C}$ & -1.3957004412 & -1.8535140066 & -1.2177927864 \\
\hline $\mathrm{C}$ & 1.3956796440 & -1.8535307308 & -1.2177929769 \\
\hline $\mathrm{H}$ & 2.4830439644 & -1.8076950568 & -1.1992301278 \\
\hline $\mathrm{H}$ & -2.4830644394 & -1.8076700371 & -1.1992295286 \\
\hline $\mathrm{C}$ & -0.7079515395 & 1.5205064165 & -1.2294718446 \\
\hline $\mathrm{C}$ & 0.7079798890 & 1.5204922715 & -1.2294727279 \\
\hline $\mathrm{C}$ & 0.6672823564 & 0.8271226892 & -3.6423288236 \\
\hline $\mathrm{C}$ & -0.6672860224 & 0.8271329174 & -3.6423290900 \\
\hline $\mathrm{C}$ & 1.4708729890 & 1.2698160747 & -2.5070355590 \\
\hline $\mathrm{C}$ & -1.4708573340 & 1.2698367708 & -2.5070271702 \\
\hline $\mathrm{H}$ & -2.4350169445 & 1.7634515984 & -2.6236415485 \\
\hline $\mathrm{H}$ & 2.4350300682 & 1.7634330468 & -2.6236650319 \\
\hline $\mathrm{H}$ & -2.4350169445 & 1.7634515984 & 2.6236415485 \\
\hline $\mathrm{C}$ & -1.4708573340 & 1.2698367708 & 2.5070271702 \\
\hline $\mathrm{C}$ & 1.4708729890 & 1.2698160747 & 2.5070355590 \\
\hline $\mathrm{C}$ & -0.7079515395 & 1.5205064165 & 1.2294718446 \\
\hline $\mathrm{C}$ & 0.7079798890 & 1.5204922715 & 1.2294727279 \\
\hline $\mathrm{H}$ & 2.4350300682 & 1.7634330468 & 2.6236650319 \\
\hline $\mathrm{C}$ & -1.3790328048 & 1.5675194760 & 0.0000000000 \\
\hline $\mathrm{C}$ & 1.3790631179 & 1.5674842766 & 0.0000000000 \\
\hline $\mathrm{H}$ & 2.4676769284 & 1.5440466255 & 0.0000000000 \\
\hline $\mathrm{H}$ & -2.4676472249 & 1.5441192073 & 0.0000000000 \\
\hline $\mathrm{C}$ & -0.7133748116 & -2.1229563175 & 0.0000000000 \\
\hline $\mathrm{C}$ & 0.7133514978 & -2.1229684957 & 0.0000000000 \\
\hline $\mathrm{C}$ & -1.3957004412 & -1.8535140066 & 1.2177927864 \\
\hline $\mathrm{C}$ & 1.3956796440 & -1.8535307308 & 1.2177929769 \\
\hline $\mathrm{H}$ & 2.4830439644 & -1.8076950568 & 1.1992301278 \\
\hline $\mathrm{H}$ & -2.4830644394 & -1.8076700371 & 1.1992295286 \\
\hline $\mathrm{C}$ & -0.7171064288 & -1.2552942673 & 2.2637265631 \\
\hline $\mathrm{C}$ & 0.7170918527 & -1.2553014721 & 2.2637256256 \\
\hline $\mathrm{C}$ & 0.6672823564 & 0.8271226892 & 3.6423288236 \\
\hline $\mathrm{C}$ & -0.6672860224 & 0.8271329174 & 3.6423290900 \\
\hline $\mathrm{C}$ & 1.4729638632 & -0.2604250719 & 3.1058961162 \\
\hline $\mathrm{C}$ & -1.4729716127 & -0.2604075300 & 3.1058898354 \\
\hline $\mathrm{H}$ & -2.4380382847 & -0.5507097174 & 3.5184910183 \\
\hline $\mathrm{H}$ & 2.4380182410 & -0.5507445867 & 3.5185141315 \\
\hline
\end{tabular}


Table S11. Cartesian Coordinates in Angstrom for the optimized structure of Hückel-[8]cyclacene containing one Ladenburg benzenoid.

\begin{tabular}{|c|c|c|c|}
\hline Atom & $\mathrm{x}$-coordinate & $\mathrm{y}$-coordinate & z-coordinate \\
\hline $\mathrm{C}$ & -3.2875576657 & 1.1978119818 & 1.4113132379 \\
\hline $\mathrm{C}$ & -3.2875092786 & 1.1977922069 & -1.4114405209 \\
\hline $\mathrm{C}$ & -1.2667966684 & 2.5221405179 & 1.4108137844 \\
\hline $\mathrm{C}$ & -1.2667495147 & 2.5221228656 & -1.4108902713 \\
\hline $\mathrm{C}$ & -2.4419026508 & 2.0900848895 & 0.7272692237 \\
\hline $\mathrm{C}$ & -2.4418783413 & 2.0900751716 & -0.7273798279 \\
\hline $\mathrm{C}$ & -1.2671027644 & -2.5219517632 & 1.4108496581 \\
\hline $\mathrm{C}$ & -1.2670555219 & -2.5219747603 & -1.4108540143 \\
\hline $\mathrm{H}$ & -1.2573603470 & -2.4784294951 & -2.4978897646 \\
\hline $\mathrm{H}$ & -1.2574443570 & -2.4783882766 & 2.4978849971 \\
\hline $\mathrm{C}$ & -0.0559244668 & 2.5160393046 & 0.7265409973 \\
\hline $\mathrm{C}$ & -0.0559002451 & 2.5160303983 & -0.7265767287 \\
\hline $\mathrm{C}$ & -0.0562297401 & -2.5160195246 & 0.7265772338 \\
\hline $\mathrm{C}$ & -0.0562053883 & -2.5160316885 & -0.7265410421 \\
\hline $\mathrm{C}$ & 1.1587150533 & 2.1417938177 & -1.4032568255 \\
\hline $\mathrm{C}$ & 1.1586680792 & 2.1418112189 & 1.4032668659 \\
\hline $\mathrm{C}$ & 1.1584582985 & -2.1419619929 & -1.4032266874 \\
\hline $\mathrm{C}$ & 1.1584110805 & -2.1419386640 & 1.4032979313 \\
\hline $\mathrm{C}$ & 3.2770496527 & 0.8274500632 & 1.4696707688 \\
\hline $\mathrm{C}$ & 3.2770968313 & 0.8274308467 & -1.4695735214 \\
\hline $\mathrm{C}$ & 2.2061199828 & 1.5709925466 & 0.7307873124 \\
\hline $\mathrm{C}$ & 2.2061442882 & 1.5709832896 & -0.7307348060 \\
\hline $\mathrm{H}$ & 1.1538410490 & 2.1326849426 & -2.4917477852 \\
\hline $\mathrm{H}$ & 1.1537567236 & 2.1327163881 & 2.4917578076 \\
\hline $\mathrm{H}$ & 1.1535851812 & -2.1328670054 & -2.4917177459 \\
\hline $\mathrm{H}$ & 1.1535005053 & -2.1328257456 & 2.4917886825 \\
\hline $\mathrm{C}$ & 2.2059346518 & -1.5712620731 & 0.7308098119 \\
\hline $\mathrm{C}$ & 4.1690775383 & -0.0002600572 & -0.6687146591 \\
\hline $\mathrm{C}$ & 2.2059591076 & -1.5712739818 & -0.7307125458 \\
\hline $\mathrm{C}$ & 3.2769538185 & -0.8278364462 & 1.4696822774 \\
\hline $\mathrm{C}$ & 4.1690563914 & -0.0002505785 & 0.6688504402 \\
\hline $\mathrm{C}$ & 3.2770012320 & -0.8278590465 & -1.4695614765 \\
\hline $\mathrm{H}$ & 3.5597563381 & -1.2136497939 & 2.4486406705 \\
\hline $\mathrm{H}$ & 3.5598330499 & -1.2136838793 & -2.4485068615 \\
\hline $\mathrm{H}$ & 3.5599737894 & 1.2132086984 & -2.4485245309 \\
\hline $\mathrm{H}$ & 3.5598974452 & 1.2132444406 & 2.4486236958 \\
\hline $\mathrm{H}$ & -1.2571433323 & 2.4785893402 & 2.4978496459 \\
\hline $\mathrm{H}$ & -1.2570592902 & 2.4785581663 & -2.4979252528 \\
\hline $\mathrm{C}$ & -3.6151991510 & 0.0002378939 & 0.7267866212 \\
\hline $\mathrm{C}$ & -2.4421273818 & -2.0897613313 & -0.7273501223 \\
\hline $\mathrm{C}$ & -3.6151737318 & 0.0002274886 & -0.7269084804 \\
\hline $\mathrm{C}$ & -3.2876952455 & -1.1973634519 & 1.4113309253 \\
\hline $\mathrm{C}$ & -2.4421517322 & -2.0897501261 & 0.7272992579 \\
\hline $\mathrm{C}$ & -3.2876465179 & -1.1973839674 & -1.4114240005 \\
\hline $\mathrm{H}$ & -3.2418738395 & -1.1728247990 & 2.4977637828 \\
\hline $\mathrm{H}$ & -3.2417882372 & -1.1728616782 & -2.4978556200 \\
\hline $\mathrm{H}$ & -3.2417424134 & 1.1732850780 & 2.4977467410 \\
\hline $\mathrm{H}$ & -3.2416571924 & 1.1732506509 & -2.4978721084 \\
\hline
\end{tabular}


Table S12. Cartesian Coordinates in Angstrom for the optimized structure of Hückel-[8]cyclacene containing two Ladenburg benzenoids.

\begin{tabular}{|c|c|c|c|}
\hline Atom & $\mathrm{x}$-coordinate & y-coordinate & z-coordinate \\
\hline $\mathrm{H}$ & -2.4367810811 & -1.2390502301 & -3.7993434781 \\
\hline $\mathrm{C}$ & -1.4709344683 & -0.8212331920 & -3.5189202085 \\
\hline $\mathrm{C}$ & 1.4709279062 & -0.8212464352 & -3.5189217842 \\
\hline $\mathrm{C}$ & -0.7190665969 & -1.4756678861 & -2.3908938378 \\
\hline $\mathrm{C}$ & 0.7190565465 & -1.4756748283 & -2.3908944898 \\
\hline $\mathrm{H}$ & 2.4367701282 & -1.2390715258 & -3.7993480997 \\
\hline $\mathrm{C}$ & -1.3947134090 & -1.8255437956 & -1.2375062175 \\
\hline $\mathrm{C}$ & 1.3947017111 & -1.8255546521 & -1.2375068457 \\
\hline $\mathrm{H}$ & 2.4828428606 & -1.7945138619 & -1.2291290542 \\
\hline $\mathrm{H}$ & -2.4828544600 & -1.7944974624 & -1.2291277797 \\
\hline $\mathrm{C}$ & -0.7141585959 & 1.9780965029 & 0.0000000000 \\
\hline $\mathrm{C}$ & 0.7141706168 & 1.9780912375 & 0.0000000000 \\
\hline $\mathrm{C}$ & -1.3947017111 & 1.8255546521 & -1.2375068457 \\
\hline $\mathrm{C}$ & 1.3947134090 & 1.8255437956 & -1.2375062175 \\
\hline $\mathrm{H}$ & 2.4828544600 & 1.7944974624 & -1.2291277797 \\
\hline $\mathrm{H}$ & -2.4828428606 & 1.7945138619 & -1.2291290542 \\
\hline $\mathrm{C}$ & -0.7190565465 & 1.4756748283 & -2.3908944898 \\
\hline $\mathrm{C}$ & 0.7190665969 & 1.4756678861 & -2.3908938378 \\
\hline $\mathrm{C}$ & 0.6674292671 & -0.0000027315 & -4.4151011736 \\
\hline $\mathrm{C}$ & -0.6674292671 & 0.0000027315 & -4.4151011736 \\
\hline $\mathrm{C}$ & 1.4709344683 & 0.8212331920 & -3.5189202085 \\
\hline $\mathrm{C}$ & -1.4709279062 & 0.8212464352 & -3.5189217842 \\
\hline $\mathrm{H}$ & -2.4367701282 & 1.2390715258 & -3.7993480997 \\
\hline $\mathrm{H}$ & 2.4367810811 & 1.2390502301 & -3.7993434781 \\
\hline $\mathrm{H}$ & -2.4367701282 & 1.2390715258 & 3.7993480997 \\
\hline $\mathrm{C}$ & -1.4709279062 & 0.8212464352 & 3.5189217842 \\
\hline $\mathrm{C}$ & 1.4709344683 & 0.8212331920 & 3.5189202085 \\
\hline $\mathrm{C}$ & -0.7190565465 & 1.4756748283 & 2.3908944898 \\
\hline $\mathrm{C}$ & 0.7190665969 & 1.4756678861 & 2.3908938378 \\
\hline $\mathrm{H}$ & 2.4367810811 & 1.2390502301 & 3.7993434781 \\
\hline $\mathrm{C}$ & -1.3947017111 & 1.8255546521 & 1.2375068457 \\
\hline $\mathrm{C}$ & 1.3947134090 & 1.8255437956 & 1.2375062175 \\
\hline $\mathrm{H}$ & 2.4828544600 & 1.7944974624 & 1.2291277797 \\
\hline $\mathrm{H}$ & -2.4828428606 & 1.7945138619 & 1.2291290542 \\
\hline $\mathrm{C}$ & -0.7141706168 & -1.9780912375 & 0.0000000000 \\
\hline $\mathrm{C}$ & 0.7141585959 & -1.9780965029 & 0.0000000000 \\
\hline $\mathrm{C}$ & -1.3947134090 & -1.8255437956 & 1.2375062175 \\
\hline $\mathrm{C}$ & 1.3947017111 & -1.8255546521 & 1.2375068457 \\
\hline $\mathrm{H}$ & 2.4828428606 & -1.7945138619 & 1.2291290542 \\
\hline $\mathrm{H}$ & -2.4828544600 & -1.7944974624 & 1.2291277797 \\
\hline $\mathrm{C}$ & -0.7190665969 & -1.4756678861 & 2.3908938378 \\
\hline $\mathrm{C}$ & 0.7190565465 & -1.4756748283 & 2.3908944898 \\
\hline $\mathrm{C}$ & 0.6674292671 & -0.0000027315 & 4.4151011736 \\
\hline $\mathrm{C}$ & -0.6674292671 & 0.0000027315 & 4.4151011736 \\
\hline $\mathrm{C}$ & 1.4709279062 & -0.8212464352 & 3.5189217842 \\
\hline $\mathrm{C}$ & -1.4709344683 & -0.8212331920 & 3.5189202085 \\
\hline $\mathrm{H}$ & -2.4367810811 & -1.2390502301 & 3.7993434781 \\
\hline $\mathrm{H}$ & 2.4367701282 & -1.2390715258 & 3.7993480997 \\
\hline
\end{tabular}


Table S13. Cartesian Coordinates in Angstrom for the optimized structure of Hückel-[9]cyclacene containing one Ladenburg benzenoid.

\begin{tabular}{|c|c|c|c|}
\hline Atom & $\mathrm{x}$-coordinate & y-coordinate & z-coordinate \\
\hline $\mathrm{C}$ & -2.9727580804 & 2.1657561934 & 1.4124619282 \\
\hline $\mathrm{C}$ & -2.9728009375 & 2.1658365345 & -1.4122404033 \\
\hline $\mathrm{C}$ & -0.6467571763 & 2.8897507527 & 1.4105630398 \\
\hline $\mathrm{C}$ & -0.6468001394 & 2.8898346264 & -1.4103704362 \\
\hline $\mathrm{C}$ & -1.8885708988 & 2.7343647598 & 0.7293703548 \\
\hline $\mathrm{C}$ & -1.8885932087 & 2.7344069656 & -0.7291494555 \\
\hline $\mathrm{C}$ & -0.6447717079 & -2.8903131922 & 1.4103978946 \\
\hline $\mathrm{C}$ & -0.6448162238 & -2.8902320472 & -1.4105355820 \\
\hline $\mathrm{H}$ & -0.6440332508 & -2.8548886394 & -2.4980482204 \\
\hline $\mathrm{H}$ & -0.6439541600 & -2.8550330371 & 2.4979125790 \\
\hline $\mathrm{C}$ & 0.5461822705 & 2.6941643117 & 0.7277700046 \\
\hline $\mathrm{C}$ & 0.5461602336 & 2.6942083034 & -0.7276252067 \\
\hline $\mathrm{C}$ & 0.5480342094 & -2.6938669716 & 0.7276159872 \\
\hline $\mathrm{C}$ & 0.5480110050 & -2.6938242980 & -0.7277798950 \\
\hline $\mathrm{C}$ & 1.7228740629 & 2.2173105910 & -1.4033323096 \\
\hline $\mathrm{C}$ & 1.7229160623 & 2.2172248005 & 1.4034127447 \\
\hline $\mathrm{C}$ & 1.7243944198 & -2.2160681879 & -1.4034573437 \\
\hline $\mathrm{C}$ & 1.7244400286 & -2.2161506777 & 1.4032829762 \\
\hline $\mathrm{C}$ & 3.7937102652 & 0.8300549605 & 1.4640458615 \\
\hline $\mathrm{C}$ & 3.7936656819 & 0.8301415162 & -1.4641104042 \\
\hline $\mathrm{C}$ & 2.7358830769 & 1.5879464860 & 0.7312718482 \\
\hline $\mathrm{C}$ & 2.7358613506 & 1.5879911975 & -0.7312599282 \\
\hline $\mathrm{H}$ & 1.7225487786 & 2.2191529621 & -2.4918491274 \\
\hline $\mathrm{H}$ & 1.7226235193 & 2.2189997697 & 2.4919296404 \\
\hline $\mathrm{H}$ & 1.7240779811 & -2.2178671879 & -2.4919745838 \\
\hline $\mathrm{H}$ & 1.7241595426 & -2.2180138990 & 2.4918001698 \\
\hline $\mathrm{C}$ & 2.7369592094 & -1.5861091824 & 0.7311799356 \\
\hline $\mathrm{C}$ & 4.6909686071 & 0.0016183401 & -0.6706693065 \\
\hline $\mathrm{C}$ & 2.7369355198 & -1.5860662455 & -0.7313503257 \\
\hline $\mathrm{C}$ & 3.7942637591 & -0.8275399487 & 1.4640001640 \\
\hline $\mathrm{C}$ & 4.6909911223 & 0.0015796732 & 0.6705284527 \\
\hline $\mathrm{C}$ & 3.7942172444 & -0.8274565423 & -1.4641600822 \\
\hline $\mathrm{H}$ & 4.0650266307 & -1.1986428447 & 2.4519959714 \\
\hline $\mathrm{H}$ & 4.0649473717 & -1.1984999746 & -2.4521871158 \\
\hline $\mathrm{H}$ & 4.0641462126 & 1.2014238929 & -2.4521152580 \\
\hline $\mathrm{H}$ & 4.0642220808 & 1.2012826433 & 2.4520628668 \\
\hline $\mathrm{H}$ & -0.6459184735 & 2.8544046050 & 2.4980753918 \\
\hline $\mathrm{H}$ & -0.6459947216 & 2.8545538175 & -2.4978849520 \\
\hline $\mathrm{C}$ & -1.8867157293 & -2.7356979137 & -0.7293044887 \\
\hline $\mathrm{C}$ & -2.9712781911 & -2.1679291453 & 1.4123390338 \\
\hline $\mathrm{C}$ & -1.8866926487 & -2.7357389531 & 0.7292147793 \\
\hline $\mathrm{C}$ & -2.9713228220 & -2.1678497292 & -1.4123625100 \\
\hline $\mathrm{H}$ & -2.9400742176 & -2.1319800644 & 2.4989868213 \\
\hline $\mathrm{H}$ & -2.9401529964 & -2.1318389276 & -2.4990092046 \\
\hline $\mathrm{H}$ & -2.9415294109 & 2.1297647828 & 2.4991073886 \\
\hline $\mathrm{H}$ & -3.9881565990 & -0.0013216076 & -2.4991395532 \\
\hline $\mathrm{C}$ & -4.0372104040 & -0.0013702275 & -1.4126035526 \\
\hline $\mathrm{C}$ & -4.0371672394 & -0.0014498656 & 1.4127351790 \\
\hline $\mathrm{C}$ & -3.7520099853 & 1.1995605511 & -0.7289832294 \\
\hline $\mathrm{C}$ & -3.7512045583 & -1.2021511390 & -0.7290511538 \\
\hline
\end{tabular}




$\begin{array}{llll}\mathrm{C} & -3.7511819408 & -1.2021923298 & 0.7291066282 \\ \mathrm{C} & -3.7519877795 & 1.1995192437 & 0.7291734713 \\ \mathrm{H} & -3.9880800746 & -0.0014629105 & 2.4992696814 \\ \mathrm{H} & -2.9416056140 & 2.1299070535 & -2.4988888329\end{array}$


Table S14. Cartesian Coordinates in Angstrom for the optimized structure of Hückel-[9]cyclacene containing two Ladenburg benzenoids.

\begin{tabular}{|c|c|c|c|}
\hline Atom & $\mathrm{x}$-coordinate & y-coordinate & z-coordinate \\
\hline $\mathrm{C}$ & 1.2145008427 & 2.3548026425 & -0.7199795509 \\
\hline $\mathrm{C}$ & 1.2144735955 & 2.3546218660 & 0.7206049679 \\
\hline $\mathrm{H}$ & 4.6014703519 & 0.4975680737 & 2.4414646501 \\
\hline $\mathrm{C}$ & 4.2117881164 & 0.1947735501 & 1.4705891808 \\
\hline $\mathrm{C}$ & 4.2118446618 & 0.1951441003 & -1.4703883423 \\
\hline $\mathrm{C}$ & 3.3474985031 & 1.1683874903 & 0.7239535824 \\
\hline $\mathrm{C}$ & 3.3475290631 & 1.1685724037 & -0.7235414630 \\
\hline $\mathrm{H}$ & 4.6015627212 & 0.4981801544 & -2.4411741516 \\
\hline $\mathrm{C}$ & 2.3688400672 & 1.8565124209 & 1.3997176495 \\
\hline $\mathrm{C}$ & 2.3688958979 & 1.8568666246 & -1.3991712150 \\
\hline $\mathrm{H}$ & 2.3512972488 & 1.8299107412 & -2.4871883728 \\
\hline $\mathrm{H}$ & 2.3511943426 & 1.8292782746 & 2.4877269154 \\
\hline $\mathrm{C}$ & 1.2461925897 & -1.8598448177 & 1.3937534914 \\
\hline $\mathrm{C}$ & 1.2462448560 & -1.8594846759 & -1.3941790535 \\
\hline $\mathrm{H}$ & 1.2427097972 & -1.8454080019 & -2.4827367346 \\
\hline $\mathrm{H}$ & 1.2426135820 & -1.8460467769 & 2.4823149271 \\
\hline $\mathrm{C}$ & 2.4403543749 & -1.6931054094 & 0.7200260937 \\
\hline $\mathrm{C}$ & 2.4403811234 & -1.6929189074 & -0.7203632825 \\
\hline $\mathrm{C}$ & 4.8109249950 & -0.8629901147 & -0.6678486067 \\
\hline $\mathrm{C}$ & 4.8108966231 & -0.8631572627 & 0.6678050884 \\
\hline $\mathrm{C}$ & 3.7002621376 & -1.3635256409 & -1.4701756336 \\
\hline $\mathrm{C}$ & 3.7002076054 & -1.3638985904 & 1.4699676823 \\
\hline $\mathrm{H}$ & 3.8416043305 & -1.8378483018 & 2.4405330186 \\
\hline $\mathrm{H}$ & 3.8416910398 & -1.8372299242 & -2.4408558235 \\
\hline $\mathrm{C}$ & -0.0000215634 & -1.9306827124 & -0.7144413930 \\
\hline $\mathrm{C}$ & -0.0000482141 & -1.9308670050 & 0.7139497226 \\
\hline $\mathrm{H}$ & -3.8417628888 & -1.8377052253 & 2.4403891036 \\
\hline $\mathrm{C}$ & -3.7003123690 & -1.3637593618 & 1.4698296675 \\
\hline $\mathrm{C}$ & -3.7002552355 & -1.3633890778 & -1.4703140963 \\
\hline $\mathrm{C}$ & -2.4404420679 & -1.6930116016 & 0.7199359831 \\
\hline $\mathrm{C}$ & -2.4404150241 & -1.6928273865 & -0.7204536738 \\
\hline $\mathrm{H}$ & -3.8416632261 & -1.8370868479 & -2.4410004565 \\
\hline $\mathrm{C}$ & -1.2463115793 & -1.8597961039 & 1.3937073311 \\
\hline $\mathrm{C}$ & -1.2462601716 & -1.8594397191 & -1.3942249867 \\
\hline $\mathrm{H}$ & -1.2426842635 & -1.8453663374 & -2.4827826667 \\
\hline $\mathrm{H}$ & -1.2427721405 & -1.8459989897 & 2.4822689230 \\
\hline $\mathrm{H}$ & -0.0000045854 & 2.4598638608 & 2.4932110072 \\
\hline $\mathrm{C}$ & 0.0000162653 & 2.5025028857 & 1.4059288683 \\
\hline $\mathrm{C}$ & 0.0000685967 & 2.5028540192 & -1.4053110516 \\
\hline $\mathrm{C}$ & -1.2144214704 & 2.3546682849 & 0.7205602905 \\
\hline $\mathrm{C}$ & -1.2143944830 & 2.3548485814 & -0.7200240966 \\
\hline $\mathrm{H}$ & 0.0000877981 & 2.4604922168 & -2.4926042405 \\
\hline $\mathrm{C}$ & -2.3688326737 & 1.8566026465 & 1.3996294258 \\
\hline $\mathrm{C}$ & -2.3687823194 & 1.8569560105 & -1.3992592322 \\
\hline $\mathrm{H}$ & -2.3511425685 & 1.8299978273 & -2.4872756471 \\
\hline $\mathrm{H}$ & -2.3512288815 & 1.8293667234 & 2.4876393373 \\
\hline $\mathrm{C}$ & -3.3474916178 & 1.1685142118 & 0.7238285700 \\
\hline $\mathrm{C}$ & -3.3474670627 & 1.1686985834 & -0.7236664286 \\
\hline $\mathrm{C}$ & -4.8109312116 & -0.8628157835 & -0.6680296372 \\
\hline $\mathrm{C}$ & -4.8109549751 & -0.8629830556 & 0.6676240217 \\
\hline
\end{tabular}




\begin{tabular}{lrrr}
$\mathrm{C}$ & -4.2117863320 & 0.1952992313 & -1.4705461261 \\
$\mathrm{C}$ & -4.2118413508 & 0.1949278324 & 1.4704314269 \\
$\mathrm{H}$ & -4.6015516151 & 0.4977359634 & 2.4412914803 \\
$\mathrm{H}$ & -4.6014566307 & 0.4983469950 & -2.4413474653 \\
\hline
\end{tabular}

Page | S15 
Table S15. Cartesian Coordinates in Angstrom for the optimized structure of Hückel-[10]cyclacene containing one Ladenburg benzenoid.

\begin{tabular}{|c|c|c|c|}
\hline Atom & $\mathrm{x}$-coordinate & y-coordinate & z-coordinate \\
\hline $\mathrm{C}$ & 2.4676185158 & 2.8848438735 & -1.4122030212 \\
\hline $\mathrm{C}$ & 2.4676246538 & 2.8849501779 & 1.4119793494 \\
\hline $\mathrm{C}$ & 0.0316428343 & 3.1187782001 & -1.4104679012 \\
\hline $\mathrm{C}$ & 0.0316484618 & 3.1188852074 & 1.4102372281 \\
\hline $\mathrm{C}$ & 1.2820636754 & 3.1755565689 & -0.7300180795 \\
\hline $\mathrm{C}$ & 1.2820667614 & 3.1756116407 & 0.7297779986 \\
\hline $\mathrm{C}$ & 0.0296943516 & -3.1189469152 & -1.4102355186 \\
\hline $\mathrm{C}$ & 0.0296999069 & -3.1188404241 & 1.4104716917 \\
\hline $\mathrm{H}$ & 0.0325331954 & -3.0914068972 & 2.4982113181 \\
\hline $\mathrm{H}$ & 0.0325232068 & -3.0915959769 & -2.4979772342 \\
\hline $\mathrm{C}$ & -1.1300047866 & 2.7921036285 & -0.7281000386 \\
\hline $\mathrm{C}$ & -1.1300021362 & 2.7921594742 & 0.7278987191 \\
\hline $\mathrm{C}$ & -1.1317374849 & -2.7914511222 & -0.7278917857 \\
\hline $\mathrm{C}$ & -1.1317343823 & -2.7913955252 & 0.7281078906 \\
\hline $\mathrm{C}$ & -2.2751545557 & 2.2431104210 & 1.4031093161 \\
\hline $\mathrm{C}$ & -2.2751587362 & 2.2430018278 & -1.4032647657 \\
\hline $\mathrm{C}$ & -2.2765294172 & -2.2415431042 & 1.4032739290 \\
\hline $\mathrm{C}$ & -2.2765364179 & -2.2416513670 & -1.4030944645 \\
\hline $\mathrm{C}$ & -4.3247010019 & 0.8264103970 & -1.4683039593 \\
\hline $\mathrm{C}$ & -4.3246961620 & 0.8265207030 & 1.4682627576 \\
\hline $\mathrm{C}$ & -3.2721296071 & 1.5907836179 & -0.7316040700 \\
\hline $\mathrm{C}$ & -3.2721277039 & 1.5908403687 & 0.7315018328 \\
\hline $\mathrm{H}$ & -2.2749705406 & 2.2464692135 & 2.4916519266 \\
\hline $\mathrm{H}$ & -2.2749782893 & 2.2462761630 & -2.4918075683 \\
\hline $\mathrm{H}$ & -2.2763569623 & -2.2448386802 & 2.4918171703 \\
\hline $\mathrm{H}$ & -2.2763691283 & -2.2450310336 & -2.4916375188 \\
\hline $\mathrm{C}$ & -3.2730809443 & -1.5887275488 & -0.7314850593 \\
\hline $\mathrm{C}$ & -5.2210668930 & 0.0016612788 & 0.6692013640 \\
\hline $\mathrm{C}$ & -3.2730769930 & -1.5886708470 & 0.7316193879 \\
\hline $\mathrm{C}$ & -4.3251840556 & -0.8237725559 & -1.4682444592 \\
\hline $\mathrm{C}$ & -5.2210694156 & 0.0016112174 & -0.6691775040 \\
\hline $\mathrm{C}$ & -4.3251770727 & -0.8236621315 & 1.4683261612 \\
\hline $\mathrm{H}$ & -4.6043066490 & -1.2026937000 & -2.4509250524 \\
\hline $\mathrm{H}$ & -4.6042948723 & -1.2025097728 & 2.4510365141 \\
\hline $\mathrm{H}$ & -4.6035895798 & 1.2056076669 & 2.4509438529 \\
\hline $\mathrm{H}$ & -4.6035975783 & 1.2054237931 & -2.4510125994 \\
\hline $\mathrm{H}$ & 0.0344565911 & 3.0913433100 & -2.4982075600 \\
\hline $\mathrm{H}$ & 0.0344664884 & 3.0915333146 & 2.4979789644 \\
\hline $\mathrm{C}$ & 1.2800818543 & -3.1764441025 & 0.7300171633 \\
\hline $\mathrm{C}$ & 2.4658257881 & -2.8866087973 & -1.4119848444 \\
\hline $\mathrm{C}$ & 1.2800791498 & -3.1764989226 & -0.7297814545 \\
\hline $\mathrm{C}$ & 2.4658308889 & -2.8865029100 & 1.4121948003 \\
\hline $\mathrm{H}$ & 2.4491462235 & -2.8506731244 & -2.4991213588 \\
\hline $\mathrm{H}$ & 2.4491545006 & -2.8504861085 & 2.4993287000 \\
\hline $\mathrm{H}$ & 2.4509053375 & 2.8488149604 & -2.4993354645 \\
\hline $\mathrm{H}$ & 4.1819199265 & 1.1905768905 & 2.5001220066 \\
\hline $\mathrm{C}$ & 4.2234152668 & 1.2080993238 & 1.4133779114 \\
\hline $\mathrm{C}$ & 4.2234088165 & 1.2079922634 & -1.4134830577 \\
\hline $\mathrm{C}$ & 3.5114333966 & 2.2107564615 & 0.7301304472 \\
\hline $\mathrm{C}$ & 3.5114302554 & 2.2107010202 & -0.7303082366 \\
\hline
\end{tabular}




\begin{tabular}{llll}
$\mathrm{H}$ & 4.1819075703 & 1.1903874196 & -2.5002255652 \\
$\mathrm{H}$ & 2.4509170781 & 2.8490028847 & 2.4991145542 \\
$\mathrm{C}$ & 4.4798882355 & -0.0014220547 & 0.7302335444 \\
$\mathrm{C}$ & 3.5100562018 & -2.2130557835 & -0.7301415296 \\
$\mathrm{C}$ & 4.4798854529 & -0.0014770997 & -0.7302480596 \\
$\mathrm{C}$ & 4.2226600589 & -1.2107341757 & 1.4134700375 \\
$\mathrm{C}$ & 3.5100588194 & -2.2130004294 & 0.7302975454 \\
$\mathrm{C}$ & 4.2226556216 & -1.2108411719 & -1.4133920624 \\
$\mathrm{H}$ & 4.1811717995 & -1.1931023387 & 2.5002127933 \\
$\mathrm{H}$ & 4.1811648615 & -1.1932915320 & -2.5001361035 \\
\hline
\end{tabular}


Table S16. Cartesian Coordinates in Angstrom for the optimized structure of Hückel-[10]cyclacene containing two Ladenburg benzenoids.

\begin{tabular}{|c|c|c|c|}
\hline Atom & $\mathrm{x}$-coordinate & y-coordinate & z-coordinate \\
\hline $\mathrm{C}$ & 1.2206530302 & 2.2296016354 & -0.7209790089 \\
\hline $\mathrm{C}$ & 1.2206539203 & 2.2296290822 & 0.7208878870 \\
\hline $\mathrm{H}$ & 4.9503501422 & 1.2298767148 & 2.4401769824 \\
\hline $\mathrm{C}$ & 4.6728005876 & 0.8202061759 & 1.4699840724 \\
\hline $\mathrm{C}$ & 4.6727982253 & 0.8201483524 & -1.4700248340 \\
\hline $\mathrm{C}$ & 3.5564546110 & 1.4926640316 & 0.7247412197 \\
\hline $\mathrm{C}$ & 3.5564536602 & 1.4926356074 & -0.7248069668 \\
\hline $\mathrm{H}$ & 4.9503461782 & 1.2297797912 & -2.4402347066 \\
\hline $\mathrm{C}$ & 2.4412332614 & 1.9261229527 & 1.3988941024 \\
\hline $\mathrm{C}$ & 2.4412315198 & 1.9260690543 & -1.3989751334 \\
\hline $\mathrm{H}$ & 2.4324597458 & 1.9041132439 & -2.4872251435 \\
\hline $\mathrm{H}$ & 2.4324631477 & 1.9042088602 & 2.4871449417 \\
\hline $\mathrm{C}$ & 1.2205510391 & -2.2296746123 & 0.7209754231 \\
\hline $\mathrm{C}$ & 1.2205500588 & -2.2297023027 & -0.7208913826 \\
\hline $\mathrm{C}$ & 2.4411446935 & -1.9261999063 & 1.3989698589 \\
\hline $\mathrm{C}$ & 2.4411427665 & -1.9262542515 & -1.3988992212 \\
\hline $\mathrm{H}$ & 2.4323723274 & -1.9043410925 & -2.4871501260 \\
\hline $\mathrm{H}$ & 2.4323754912 & -1.9042447143 & 2.4872199518 \\
\hline $\mathrm{C}$ & 3.5563862527 & -1.4928183147 & 0.7248003683 \\
\hline $\mathrm{C}$ & 3.5563853289 & -1.4928469995 & -0.7247480589 \\
\hline $\mathrm{C}$ & 5.5712052351 & -0.0001504831 & -0.6679698017 \\
\hline $\mathrm{C}$ & 5.5712062882 & -0.0001243160 & 0.6679602695 \\
\hline $\mathrm{C}$ & 4.6727611068 & -0.8204400414 & -1.4699923866 \\
\hline $\mathrm{C}$ & 4.6727626622 & -0.8203818192 & 1.4700167539 \\
\hline $\mathrm{H}$ & 4.9502934017 & -1.2300262072 & 2.4402260436 \\
\hline $\mathrm{H}$ & 4.9502905201 & -1.2301228366 & -2.4401858224 \\
\hline $\mathrm{C}$ & -0.0000532509 & -2.3179480973 & -1.4043122303 \\
\hline $\mathrm{C}$ & -0.0000514425 & -2.3178940180 & 1.4044013418 \\
\hline $\mathrm{H}$ & -0.0000500607 & -2.2926379925 & 2.4923005734 \\
\hline $\mathrm{H}$ & -0.0000532848 & -2.2927349217 & -2.4922124667 \\
\hline $\mathrm{C}$ & -1.2206515294 & -2.2296449662 & -0.7208898492 \\
\hline $\mathrm{C}$ & -1.2206506454 & -2.2296172242 & 0.7209770479 \\
\hline $\mathrm{H}$ & -4.9503441973 & -1.2297957380 & 2.4402323125 \\
\hline $\mathrm{C}$ & -4.6727959258 & -0.8201638716 & 1.4700227013 \\
\hline $\mathrm{C}$ & -4.6727981091 & -0.8202221080 & -1.4699862117 \\
\hline $\mathrm{C}$ & -3.5564512723 & -1.4926512061 & 0.7248048906 \\
\hline $\mathrm{C}$ & -3.5564522238 & -1.4926798992 & -0.7247432953 \\
\hline $\mathrm{H}$ & -4.9503473648 & -1.2298922546 & -2.4401793637 \\
\hline $\mathrm{C}$ & -2.4412291323 & -1.9260845029 & 1.3989731198 \\
\hline $\mathrm{C}$ & -2.4412308742 & -1.9261389685 & -1.3988961166 \\
\hline $\mathrm{H}$ & -2.4324607735 & -1.9042250763 & -2.4871469598 \\
\hline $\mathrm{H}$ & -2.4324573402 & -1.9041284794 & 2.4872231259 \\
\hline $\mathrm{H}$ & 0.0000557282 & 2.2927186246 & 2.4922105290 \\
\hline $\mathrm{C}$ & 0.0000556573 & 2.3179320558 & 1.4043102991 \\
\hline $\mathrm{C}$ & 0.0000538128 & 2.3178785758 & -1.4044032727 \\
\hline $\mathrm{C}$ & -1.2205476591 & 2.2296864042 & 0.7208894481 \\
\hline $\mathrm{C}$ & -1.2205486628 & 2.2296590316 & -0.7209773575 \\
\hline $\mathrm{H}$ & 0.0000524114 & 2.2926227779 & -2.4923025101 \\
\hline $\mathrm{C}$ & -2.4411403582 & 1.9262381917 & 1.3988972446 \\
\hline $\mathrm{C}$ & -2.4411423278 & 1.9261844844 & -1.3989718355 \\
\hline
\end{tabular}




\begin{tabular}{llll}
$\mathrm{H}$ & -2.4323731538 & 1.9042295232 & -2.4872219330 \\
$\mathrm{H}$ & -2.4323698911 & 1.9043248087 & 2.4871481451 \\
$\mathrm{C}$ & -3.5563829291 & 1.4928310984 & 0.7247460215 \\
$\mathrm{C}$ & -3.5563838728 & 1.4928027240 & -0.7248024066 \\
$\mathrm{C}$ & -5.5712037475 & 0.0001085909 & -0.6679626542 \\
$\mathrm{C}$ & -5.5712030139 & 0.0001347417 & 0.6679674145 \\
$\mathrm{C}$ & -4.6727601854 & 0.8203663134 & -1.4700188749 \\
$\mathrm{C}$ & -4.6727587986 & 0.8204240467 & 1.4699902796 \\
$\mathrm{H}$ & -4.9502885195 & 1.2301072469 & 2.4401834664 \\
$\mathrm{H}$ & -4.9502906199 & 1.2300103334 & -2.4402283978 \\
\hline
\end{tabular}


Table S17. Cartesian Coordinates in Angstrom for the optimized structure of Hückel-[11]cyclacene containing one Ladenburg benzenoid.

\begin{tabular}{|c|c|c|c|}
\hline Atom & $\mathrm{x}$-coordinate & y-coordinate & z-coordinate \\
\hline $\mathrm{C}$ & 1.8781847765 & 3.4103053400 & -1.4120852396 \\
\hline $\mathrm{C}$ & 1.8781794600 & 3.4104378421 & 1.4117668402 \\
\hline $\mathrm{C}$ & -0.5727437372 & 3.2903668067 & -1.4104841602 \\
\hline $\mathrm{C}$ & -0.5727494639 & 3.2905052254 & 1.4101673861 \\
\hline $\mathrm{C}$ & 0.6619128045 & 3.4918248746 & -0.7309507076 \\
\hline $\mathrm{C}$ & 0.6619102472 & 3.4918947990 & 0.7306193968 \\
\hline $\mathrm{C}$ & -0.5739652414 & -3.2903284142 & -1.4101700425 \\
\hline $\mathrm{C}$ & -0.5739647332 & -3.2901899932 & 1.4104819818 \\
\hline $\mathrm{H}$ & -0.5697381731 & -3.2682606708 & 2.4983789455 \\
\hline $\mathrm{H}$ & -0.5697393768 & -3.2685058980 & -2.4980691160 \\
\hline $\mathrm{C}$ & -1.7056081252 & 2.8768388737 & -0.7286818225 \\
\hline $\mathrm{C}$ & -1.7056114247 & 2.8769117716 & 0.7284007034 \\
\hline $\mathrm{C}$ & -1.7066733752 & -2.8763096842 & -0.7284053372 \\
\hline $\mathrm{C}$ & -1.7066727578 & -2.8762367665 & 0.7286773425 \\
\hline $\mathrm{C}$ & -2.8253390927 & 2.2794171237 & 1.4032350005 \\
\hline $\mathrm{C}$ & -2.8253319435 & 2.2792749513 & -1.4034613444 \\
\hline $\mathrm{C}$ & -2.8261742081 & -2.2782490294 & 1.4034533857 \\
\hline $\mathrm{C}$ & -2.8261761730 & -2.2783912496 & -1.4032403747 \\
\hline $\mathrm{C}$ & -4.8482612768 & 0.8270111222 & -1.4654986590 \\
\hline $\mathrm{C}$ & -4.8482661446 & 0.8271543489 & 1.4654083579 \\
\hline $\mathrm{C}$ & -3.8030324798 & 1.5988461958 & -0.7318544320 \\
\hline $\mathrm{C}$ & -3.8030363547 & 1.5989204340 & 0.7316918671 \\
\hline $\mathrm{H}$ & -2.8285424602 & 2.2884820560 & 2.4917507492 \\
\hline $\mathrm{H}$ & -2.8285307933 & 2.2882299510 & -2.4919779520 \\
\hline $\mathrm{H}$ & -2.8293852041 & -2.2872144899 & 2.4919701914 \\
\hline $\mathrm{H}$ & -2.8293876651 & -2.2874667621 & -2.4917563231 \\
\hline $\mathrm{C}$ & -3.8036111797 & -1.5975149084 & -0.7316998571 \\
\hline $\mathrm{C}$ & -5.7465165920 & 0.0010834821 & 0.6702134519 \\
\hline $\mathrm{C}$ & -3.8036099995 & -1.5974406601 & 0.7318453974 \\
\hline $\mathrm{C}$ & -4.8485558983 & -0.8253702178 & -1.4654198329 \\
\hline $\mathrm{C}$ & -5.7465153538 & 0.0010187123 & -0.6702255275 \\
\hline $\mathrm{C}$ & -4.8485563857 & -0.8252270019 & 1.4654892507 \\
\hline $\mathrm{H}$ & -5.1213624271 & -1.1963255012 & -2.4528696524 \\
\hline $\mathrm{H}$ & -5.1213632815 & -1.1960864209 & 2.4529750643 \\
\hline $\mathrm{H}$ & -5.1209411587 & 1.1982072571 & 2.4528574699 \\
\hline $\mathrm{H}$ & -5.1209330824 & 1.1979681743 & -2.4529847507 \\
\hline $\mathrm{H}$ & -0.5685232924 & 3.2684392013 & -2.4983811584 \\
\hline $\mathrm{H}$ & -0.5685338113 & 3.2686844163 & 2.4980665226 \\
\hline $\mathrm{C}$ & 0.6606179776 & -3.4921130174 & 0.7309506444 \\
\hline $\mathrm{C}$ & 1.8769202297 & -3.4111802633 & -1.4117642825 \\
\hline $\mathrm{C}$ & 0.6606180972 & -3.4921829261 & -0.7306195381 \\
\hline $\mathrm{C}$ & 1.8769203955 & -3.4110478408 & 1.4120870171 \\
\hline $\mathrm{H}$ & 1.8694617074 & -3.3778198168 & -2.4991137833 \\
\hline $\mathrm{H}$ & 1.8694629287 & -3.3775842219 & 2.4994333614 \\
\hline $\mathrm{H}$ & 1.8707182105 & 3.3768508892 & -2.4994318349 \\
\hline $\mathrm{H}$ & 3.9960323432 & 2.2273600908 & 2.5003753967 \\
\hline $\mathrm{C}$ & 4.0257514441 & 2.2535400057 & 1.4133888650 \\
\hline $\mathrm{C}$ & 4.0257564578 & 2.2534101042 & -1.4135915340 \\
\hline $\mathrm{C}$ & 3.0491132881 & 2.9959428867 & 0.7310718648 \\
\hline $\mathrm{C}$ & 3.0491159207 & 2.9958751202 & -0.7313463846 \\
\hline
\end{tabular}




\begin{tabular}{lccc}
$\mathrm{H}$ & 3.9960422446 & 2.2271303293 & -2.5005758274 \\
$\mathrm{H}$ & 1.8707078157 & 3.3770866081 & 2.4991165987 \\
$\mathrm{C}$ & 3.0480102813 & -2.9971278543 & -0.7310679019 \\
$\mathrm{C}$ & 4.0249253185 & -2.2549536976 & 1.4135973053 \\
$\mathrm{C}$ & 3.0480102864 & -2.9970601229 & 0.7313510752 \\
$\mathrm{C}$ & 4.0249253360 & -2.2550835806 & -1.4133824290 \\
$\mathrm{H}$ & 3.9952230650 & -2.2286673618 & 2.5005818061 \\
$\mathrm{H}$ & 3.9952221347 & -2.2288972521 & -2.5003692395 \\
$\mathrm{H}$ & 4.8865387410 & -0.0010467147 & -2.5008462784 \\
$\mathrm{C}$ & 4.9279930732 & -0.0010026519 & -1.4138816880 \\
$\mathrm{C}$ & 4.9279905939 & -0.0008722558 & 1.4138894007 \\
$\mathrm{C}$ & 4.6884825505 & 1.2085598638 & -0.7315340477 \\
$\mathrm{C}$ & 4.6880352203 & -1.2104147540 & -0.7314223720 \\
$\mathrm{C}$ & 4.6880348297 & -1.2103472747 & 0.7315410581 \\
$\mathrm{C}$ & 4.6884803398 & 1.2086273517 & 0.7314298938 \\
$\mathrm{H}$ & 4.8865342983 & -0.0008149119 & 2.5008539383 \\
\hline
\end{tabular}


Table S18. Cartesian Coordinates in Angstrom for the optimized structure of Hückel-[11]cyclacene containing two Ladenburg benzenoids.

\begin{tabular}{|c|c|c|c|}
\hline Atom & $\mathrm{x}$-coordinate & y-coordinate & z-coordinate \\
\hline $\mathrm{C}$ & -0.0001842165 & 2.9557970596 & -0.7250402407 \\
\hline $\mathrm{C}$ & -0.0001824505 & 2.9556881218 & 0.7255035708 \\
\hline $\mathrm{C}$ & 1.2285881141 & 2.8211726402 & -1.4071031346 \\
\hline $\mathrm{C}$ & 1.2285919060 & 2.8209606229 & 1.4075425418 \\
\hline $\mathrm{H}$ & 1.2233005486 & 2.7923834860 & 2.4952604214 \\
\hline $\mathrm{H}$ & 1.2232936060 & 2.7927593711 & -2.4948252746 \\
\hline $\mathrm{C}$ & 2.3868846878 & 2.4577649414 & -0.7238206973 \\
\hline $\mathrm{C}$ & 2.3868866116 & 2.4576548583 & 0.7242023279 \\
\hline $\mathrm{H}$ & 5.6753571458 & 0.4559842197 & 2.4444842393 \\
\hline $\mathrm{C}$ & 5.3043601686 & 0.1437740555 & 1.4691385077 \\
\hline $\mathrm{C}$ & 5.3043606140 & 0.1440056501 & -1.4691200909 \\
\hline $\mathrm{C}$ & 4.4225425129 & 1.1002785371 & 0.7274100676 \\
\hline $\mathrm{C}$ & 4.4225430660 & 1.1003932687 & -0.7272413044 \\
\hline $\mathrm{H}$ & 5.6753577836 & 0.4563700059 & -2.4444164830 \\
\hline $\mathrm{C}$ & 3.4890650943 & 1.8424124095 & 1.4018720912 \\
\hline $\mathrm{C}$ & 3.4890631961 & 1.8426296577 & -1.4015871079 \\
\hline $\mathrm{H}$ & 3.4791459706 & 1.8327486375 & -2.4899874340 \\
\hline $\mathrm{H}$ & 3.4791494551 & 1.8323637289 & 2.4902709034 \\
\hline $\mathrm{C}$ & 1.2231646353 & -2.3022562413 & 0.7207751527 \\
\hline $\mathrm{C}$ & 1.2231630645 & -2.3021350376 & -0.7211417876 \\
\hline $\mathrm{C}$ & 2.4687185885 & -2.1199291540 & 1.3979828835 \\
\hline $\mathrm{C}$ & 2.4687163214 & -2.1196968213 & -1.3983206129 \\
\hline $\mathrm{H}$ & 2.4653073744 & -2.1172128025 & -2.4869564916 \\
\hline $\mathrm{H}$ & 2.4653106497 & -2.1176267569 & 2.4866191971 \\
\hline $\mathrm{C}$ & 3.6345879019 & -1.8463355650 & 0.7256942355 \\
\hline $\mathrm{C}$ & 3.6345868353 & -1.8462165246 & -0.7259881531 \\
\hline $\mathrm{C}$ & 5.9555083163 & -0.8848270845 & -0.6683808016 \\
\hline $\mathrm{C}$ & 5.9555061313 & -0.8849322283 & 0.6682369407 \\
\hline $\mathrm{C}$ & 4.8704773263 & -1.4385869482 & -1.4695043260 \\
\hline $\mathrm{C}$ & 4.8704774908 & -1.4388216284 & 1.4692740695 \\
\hline $\mathrm{H}$ & 5.0349797959 & -1.8952497036 & 2.4445528398 \\
\hline $\mathrm{H}$ & 5.0349765619 & -1.8948624993 & -2.4448548308 \\
\hline $\mathrm{C}$ & 0.0001400155 & -2.3580396061 & -1.4038192532 \\
\hline $\mathrm{C}$ & 0.0001433748 & -2.3582754257 & 1.4034460180 \\
\hline $\mathrm{H}$ & 0.0001436645 & -2.3438564488 & 2.4916023916 \\
\hline $\mathrm{H}$ & 0.0001376801 & -2.3434367940 & -2.4919730578 \\
\hline $\mathrm{C}$ & -1.2228881631 & -2.3022843982 & -0.7211387426 \\
\hline $\mathrm{C}$ & -1.2228862729 & -2.3024057100 & 0.7207779029 \\
\hline $\mathrm{H}$ & -5.0347463612 & -1.8958721909 & 2.4445652657 \\
\hline $\mathrm{C}$ & -4.8703033724 & -1.4394240980 & 1.4692857752 \\
\hline $\mathrm{C}$ & -4.8703105042 & -1.4391891259 & -1.4694924242 \\
\hline $\mathrm{C}$ & -3.6343660621 & -1.8467831886 & 0.7257025953 \\
\hline $\mathrm{C}$ & -3.6343685130 & -1.8466639662 & -0.7259790658 \\
\hline $\mathrm{H}$ & -5.0347553384 & -1.8954847813 & -2.4448427773 \\
\hline $\mathrm{C}$ & -2.4684613415 & -2.1202327793 & 1.3979885661 \\
\hline $\mathrm{C}$ & -2.4684658128 & -2.1200000788 & -1.3983145001 \\
\hline $\mathrm{H}$ & -2.4650602037 & -2.1175153343 & -2.4869503890 \\
\hline $\mathrm{H}$ & -2.4650514397 & -2.1179298359 & 2.4866248747 \\
\hline $\mathrm{H}$ & -1.2236413368 & 2.7922346411 & 2.4952635943 \\
\hline $\mathrm{C}$ & -1.2289385943 & 2.8208110737 & 1.4075457120 \\
\hline
\end{tabular}




\begin{tabular}{llll}
$\mathrm{C}$ & -1.2289416528 & 2.8210230064 & -1.4071003259 \\
$\mathrm{C}$ & -2.3871900001 & 2.4573622428 & 0.7242082743 \\
$\mathrm{C}$ & -2.3871915836 & 2.4574722616 & -0.7238150195 \\
$\mathrm{H}$ & -1.2236465911 & 2.7926103848 & -2.4948224866 \\
$\mathrm{C}$ & -3.4892896268 & 1.8419804740 & 1.4018808853 \\
$\mathrm{C}$ & -3.4892945476 & 1.8421976268 & -1.4015788993 \\
$\mathrm{H}$ & -3.4793798778 & 1.8323195092 & -2.4899792958 \\
$\mathrm{H}$ & -3.4793712942 & 1.8319348206 & 2.4902797185 \\
$\mathrm{C}$ & -4.4226743176 & 1.0997289469 & 0.7274208404 \\
$\mathrm{C}$ & -4.4226784267 & 1.0998436683 & -0.7272305084 \\
$\mathrm{C}$ & -5.9554065398 & -0.8855599728 & -0.6683663132 \\
$\mathrm{C}$ & -5.9554009334 & -0.8856651484 & 0.6682515769 \\
$\mathrm{C}$ & -5.3043846258 & 0.1433508332 & -1.4691072032 \\
$\mathrm{C}$ & -5.3043769469 & 0.1431192544 & 1.4691514946 \\
$\mathrm{H}$ & -5.6754087347 & 0.4552850582 & 2.4444980086 \\
$\mathrm{H}$ & -5.6754216356 & 0.4556710636 & -2.4444023976 \\
\hline
\end{tabular}


Table S19. Cartesian Coordinates in Angstrom for the optimized structure of Hückel-[12]cyclacene containing one Ladenburg benzenoid.

\begin{tabular}{|c|c|c|c|}
\hline Atom & $\mathrm{x}$-coordinate & y-coordinate & z-coordinate \\
\hline $\mathrm{H}$ & 1.2632308748 & 3.7830951473 & -2.4989405522 \\
\hline $\mathrm{C}$ & 1.2652934776 & 3.8116836192 & -1.4113479702 \\
\hline $\mathrm{C}$ & 1.2652421684 & 3.8115623770 & 1.4117298434 \\
\hline $\mathrm{C}$ & 2.4911717956 & 3.6091618453 & -0.7314404987 \\
\hline $\mathrm{C}$ & 2.4911451847 & 3.6090991884 & 0.7318494201 \\
\hline $\mathrm{H}$ & 1.2631397450 & 3.7828796813 & 2.4993198899 \\
\hline $\mathrm{C}$ & 3.6063728364 & 3.1017260844 & -1.4131540866 \\
\hline $\mathrm{C}$ & 3.6063206462 & 3.1016045869 & 1.4135610816 \\
\hline $\mathrm{H}$ & 3.5850902934 & 3.0703903872 & 2.5006584034 \\
\hline $\mathrm{H}$ & 3.5851838263 & 3.0706052186 & -2.5002548792 \\
\hline $\mathrm{C}$ & 4.5313253725 & 2.2801186718 & -0.7318056570 \\
\hline $\mathrm{C}$ & 4.5312979237 & 2.2800552259 & 0.7321765552 \\
\hline $\mathrm{C}$ & 5.3580366763 & -0.0013010524 & 0.7322144299 \\
\hline $\mathrm{C}$ & 5.3580655619 & -0.0012372385 & -0.7320097770 \\
\hline $\mathrm{C}$ & 5.1467945682 & 1.2144758219 & 1.4141739921 \\
\hline $\mathrm{C}$ & 5.1468486613 & 1.2145987220 & -1.4138717902 \\
\hline $\mathrm{H}$ & 5.1124185498 & 1.2025995860 & -2.5010015603 \\
\hline $\mathrm{H}$ & 5.1123221016 & 1.2023821708 & 2.5013014067 \\
\hline $\mathrm{C}$ & 0.0498609227 & 3.7328718185 & 0.7310908080 \\
\hline $\mathrm{C}$ & 0.0498874395 & 3.7329350525 & -0.7307599200 \\
\hline $\mathrm{C}$ & -1.1608879746 & 3.4175217281 & 1.4104351611 \\
\hline $\mathrm{C}$ & -1.1608370055 & 3.4176451803 & -1.4101758200 \\
\hline $\mathrm{H}$ & -1.1559130414 & 3.3990749710 & -2.4981259536 \\
\hline $\mathrm{H}$ & -1.1560038862 & 3.3988556111 & 2.4983838217 \\
\hline $\mathrm{C}$ & -2.2654958083 & 2.9359775822 & 0.7286469319 \\
\hline $\mathrm{C}$ & -2.2654698952 & 2.9360421452 & -0.7284698292 \\
\hline $\mathrm{H}$ & -5.6477352255 & 1.2003424731 & -2.4524403591 \\
\hline $\mathrm{C}$ & -5.3703871191 & 0.8252975846 & -1.4677894554 \\
\hline $\mathrm{C}$ & -5.3704367189 & 0.8251676164 & 1.4676692359 \\
\hline $\mathrm{C}$ & -4.3292877588 & 1.6023719484 & -0.7318441287 \\
\hline $\mathrm{C}$ & -4.3293124465 & 1.6023053426 & 0.7318289353 \\
\hline $\mathrm{H}$ & -5.6478158455 & 1.2001215439 & 2.4523461121 \\
\hline $\mathrm{C}$ & -3.3632511553 & 2.2984280739 & -1.4031619263 \\
\hline $\mathrm{C}$ & -3.3632998417 & 2.2983015344 & 1.4032431636 \\
\hline $\mathrm{H}$ & -3.3674368134 & 2.3086753191 & 2.4917691310 \\
\hline $\mathrm{H}$ & -3.3673495635 & 2.3089010357 & -2.4916871323 \\
\hline $\mathrm{H}$ & -1.1574754869 & -3.3983678254 & -2.4984280224 \\
\hline $\mathrm{C}$ & -1.1624137039 & -3.4170459134 & -1.4104800068 \\
\hline $\mathrm{C}$ & -1.1624692997 & -3.4171806805 & 1.4101318234 \\
\hline $\mathrm{C}$ & -2.2668305348 & -2.9350092044 & -0.7287315352 \\
\hline $\mathrm{C}$ & -2.2668593362 & -2.9350792676 & 0.7283853667 \\
\hline $\mathrm{H}$ & -1.1575753876 & -3.3986068460 & 2.4980818446 \\
\hline $\mathrm{C}$ & -3.3643177005 & -2.2968256243 & -1.4033661161 \\
\hline $\mathrm{C}$ & -3.3643728091 & -2.2969600244 & 1.4030378604 \\
\hline $\mathrm{H}$ & -3.3685227876 & -2.3074444676 & 2.4915630661 \\
\hline $\mathrm{H}$ & -3.3684263969 & -2.3072074783 & -2.4918923926 \\
\hline $\mathrm{C}$ & -4.3300205296 & -1.6003671227 & -0.7319858006 \\
\hline $\mathrm{C}$ & -4.3300489910 & -1.6004367870 & 0.7316859513 \\
\hline $\mathrm{C}$ & -6.2684600557 & 0.0013799901 & 0.6693388813 \\
\hline $\mathrm{C}$ & -6.2684376268 & 0.0014396496 & -0.6695618291 \\
\hline
\end{tabular}




\begin{tabular}{lccc}
$\mathrm{C}$ & -5.3708116907 & -0.8228867844 & 1.4675967105 \\
$\mathrm{C}$ & -5.3707587465 & -0.8227529046 & -1.4678636053 \\
$\mathrm{H}$ & -5.6482774311 & -1.1975841651 & -2.4525483522 \\
$\mathrm{H}$ & -5.6483635313 & -1.1978030202 & 2.4522396724 \\
$\mathrm{C}$ & 0.0481384475 & -3.7330164115 & 0.7307602251 \\
$\mathrm{C}$ & 0.0481667684 & -3.7329471915 & -0.7310904164 \\
$\mathrm{C}$ & 1.2634835490 & -3.8123167802 & 1.4113929099 \\
$\mathrm{C}$ & 1.2635377480 & -3.8121855439 & -1.4116839819 \\
$\mathrm{H}$ & 1.2614892551 & -3.7834989123 & -2.4992738913 \\
$\mathrm{H}$ & 1.2613931780 & -3.7837320040 & 2.4989855102 \\
$\mathrm{C}$ & 2.4894802826 & -3.6103568737 & 0.7315314365 \\
$\mathrm{C}$ & 2.4895085828 & -3.6102906075 & -0.7317570083 \\
$\mathrm{H}$ & 5.1118863917 & -1.2048095642 & -2.5011067174 \\
$\mathrm{C}$ & 5.1463071079 & -1.2169198340 & -1.4139776645 \\
$\mathrm{C}$ & 5.1462500981 & -1.2170432225 & 1.4140678593 \\
$\mathrm{C}$ & 4.5302929902 & -2.2822168045 & -0.7320049845 \\
$\mathrm{C}$ & 4.5302638120 & -2.2822811776 & 0.7319777527 \\
$\mathrm{H}$ & 5.1117866010 & -1.2050278676 & 2.5011966766 \\
$\mathrm{C}$ & 3.6049495606 & -3.1033247533 & -1.4134258587 \\
$\mathrm{C}$ & 3.6048941373 & -3.1034510785 & 1.4132891626 \\
$\mathrm{H}$ & 3.5836771160 & -3.0723183814 & 2.5003890178 \\
$\mathrm{H}$ & 3.5837745741 & -3.0720952432 & -2.5005236915 \\
\hline
\end{tabular}


Table S20. Cartesian Coordinates in Angstrom for the optimized structure of Hückel-[12]cyclacene containing two Ladenburg benzenoids.

\begin{tabular}{|c|c|c|c|}
\hline Atom & $\mathrm{x}$-coordinate & y-coordinate & z-coordinate \\
\hline $\mathrm{C}$ & 0.0008700954 & 2.7696036045 & -0.7254375488 \\
\hline $\mathrm{C}$ & 0.0008622123 & 2.7695922584 & 0.7254761182 \\
\hline $\mathrm{C}$ & 1.2342874535 & 2.6831035914 & -1.4063585479 \\
\hline $\mathrm{C}$ & 1.2342721484 & 2.6830818426 & 1.4064091588 \\
\hline $\mathrm{H}$ & 1.2318422369 & 2.6640131175 & 2.4944285281 \\
\hline $\mathrm{H}$ & 1.2318694418 & 2.6640518156 & -2.4943782215 \\
\hline $\mathrm{C}$ & 2.4252772587 & 2.4467832218 & -0.7242513687 \\
\hline $\mathrm{C}$ & 2.4252693750 & 2.4467720683 & 0.7243112703 \\
\hline $\mathrm{H}$ & 6.0689748574 & 1.2187500331 & 2.4438085089 \\
\hline $\mathrm{C}$ & 5.7924257858 & 0.8187756846 & 1.4691717175 \\
\hline $\mathrm{C}$ & 5.7924416586 & 0.8187979707 & -1.4690995242 \\
\hline $\mathrm{C}$ & 4.6941027954 & 1.5182321381 & 0.7279424495 \\
\hline $\mathrm{C}$ & 4.6941107850 & 1.5182433515 & -0.7278716041 \\
\hline $\mathrm{H}$ & 6.0690006639 & 1.2187862940 & -2.4437277553 \\
\hline $\mathrm{C}$ & 3.6184359219 & 2.0336981119 & 1.4015147414 \\
\hline $\mathrm{C}$ & 3.6184513597 & 2.0337197458 & -1.4014479204 \\
\hline $\mathrm{H}$ & 3.6123249991 & 2.0238806137 & -2.4898134016 \\
\hline $\mathrm{H}$ & 3.6122973122 & 2.0238423109 & 2.4898799996 \\
\hline $\mathrm{H}$ & 1.2301812411 & -2.6648964804 & 2.4943889599 \\
\hline $\mathrm{C}$ & 1.2326024430 & -2.6839640507 & 1.4063699136 \\
\hline $\mathrm{C}$ & 1.2326177502 & -2.6839424642 & -1.4063993746 \\
\hline $\mathrm{C}$ & 2.4237636059 & -2.4484698932 & 0.7242754943 \\
\hline $\mathrm{C}$ & 2.4237714418 & -2.4484589359 & -0.7242882912 \\
\hline $\mathrm{H}$ & 1.2302086586 & -2.6648581900 & -2.4944181409 \\
\hline $\mathrm{C}$ & 3.6172010689 & -2.0361856032 & 1.4014851089 \\
\hline $\mathrm{C}$ & 3.6172163351 & -2.0361648163 & -1.4014787290 \\
\hline $\mathrm{H}$ & 3.6110957143 & -2.0263019830 & -2.4898441476 \\
\hline $\mathrm{H}$ & 3.6110684305 & -2.0263388426 & 2.4898506027 \\
\hline $\mathrm{C}$ & 4.6931947513 & -1.5213935360 & 0.7279207183 \\
\hline $\mathrm{C}$ & 4.6932026888 & -1.5213827493 & -0.7278949552 \\
\hline $\mathrm{C}$ & 6.6913300230 & -0.0021650080 & -0.6684178906 \\
\hline $\mathrm{C}$ & 6.6913228674 & -0.0021749407 & 0.6684869739 \\
\hline $\mathrm{C}$ & 5.7919578484 & -0.8225855622 & -1.4691112868 \\
\hline $\mathrm{C}$ & 5.7919422480 & -0.8226070880 & 1.4691590559 \\
\hline $\mathrm{H}$ & 6.0682552533 & -1.2227595381 & 2.4437889563 \\
\hline $\mathrm{H}$ & 6.0682805509 & -1.2227228023 & -2.4437446873 \\
\hline $\mathrm{C}$ & -0.0008673939 & -2.7696126297 & 0.7254346824 \\
\hline $\mathrm{C}$ & -0.0008595041 & -2.7696014689 & -0.7254789852 \\
\hline $\mathrm{C}$ & -1.2342694405 & -2.6830911383 & -1.4064120377 \\
\hline $\mathrm{C}$ & -1.2342847508 & -2.6831125385 & 1.4063556709 \\
\hline $\mathrm{H}$ & -1.2318667387 & -2.6640606297 & 2.4943753424 \\
\hline $\mathrm{H}$ & -1.2318395295 & -2.6640225523 & -2.4944314090 \\
\hline $\mathrm{C}$ & -2.4252666687 & -2.4467812767 & -0.7243141779 \\
\hline $\mathrm{C}$ & -2.4252745517 & -2.4467922548 & 0.7242484613 \\
\hline $\mathrm{H}$ & -6.0689981683 & -1.2187954816 & 2.4437245987 \\
\hline $\mathrm{C}$ & -5.7924390155 & -0.8188069997 & 1.4690964709 \\
\hline $\mathrm{C}$ & -5.7924230154 & -0.8187849071 & -1.4691747586 \\
\hline $\mathrm{C}$ & -4.6941080812 & -1.5182523993 & 0.7278686041 \\
\hline $\mathrm{C}$ & -4.6941000823 & -1.5182413266 & -0.7279454502 \\
\hline $\mathrm{H}$ & -6.0689719194 & -1.2187590496 & -2.4438116769 \\
\hline
\end{tabular}




\begin{tabular}{lllc}
$\mathrm{C}$ & -3.6184486551 & -2.0337287076 & 1.4014449699 \\
$\mathrm{C}$ & -3.6184332111 & -2.0337073889 & -1.4015176925 \\
$\mathrm{H}$ & -3.6122946015 & -2.0238517002 & -2.4898829514 \\
$\mathrm{H}$ & -3.6123222912 & -2.0238894599 & 2.4898104502 \\
$\mathrm{H}$ & -1.2302059426 & 2.6648487424 & 2.4944152654 \\
$\mathrm{C}$ & -1.2326150388 & 2.6839331642 & 1.4063965015 \\
$\mathrm{C}$ & -1.2325997434 & 2.6839551233 & -1.4063727888 \\
$\mathrm{C}$ & -2.4237687298 & 2.4484497284 & 0.7242853913 \\
$\mathrm{C}$ & -2.4237609053 & 2.4484608819 & -0.7242783950 \\
$\mathrm{H}$ & -1.2301785424 & 2.6648876979 & -2.4943918372 \\
$\mathrm{C}$ & -3.6172136214 & 2.0361555277 & 1.4014757872 \\
$\mathrm{C}$ & -3.6171983710 & 2.0361766804 & -1.4014880522 \\
$\mathrm{H}$ & -3.6110657430 & 2.0263300537 & -2.4898535470 \\
$\mathrm{H}$ & -3.6110929898 & 2.0262925618 & 2.4898412047 \\
$\mathrm{C}$ & -4.6931999842 & 1.5213735654 & 0.7278919626 \\
$\mathrm{C}$ & -4.6931920469 & 1.5213845165 & -0.7279237117 \\
$\mathrm{C}$ & -6.6913200589 & 0.0021658291 & -0.6684901480 \\
$\mathrm{C}$ & -6.6913274006 & 0.0021558937 & 0.6684147193 \\
$\mathrm{C}$ & -5.7919394880 & 0.8225981050 & -1.4691620898 \\
$\mathrm{C}$ & -5.7919552039 & 0.8225763515 & 1.4691082380 \\
$\mathrm{H}$ & -6.0682780538 & 1.2227137555 & 2.4437415338 \\
$\mathrm{H}$ & -6.0682523334 & 1.2227503480 & -2.4437921154 \\
\hline
\end{tabular}


Table S21. Cartesian Coordinates in Angstrom for the optimized structure of Hückel-[13]cyclacene containing one Ladenburg benzenoid.

\begin{tabular}{|c|c|c|c|}
\hline Atom & $\mathrm{x}$-coordinate & $\mathrm{y}$-coordinate & z-coordinate \\
\hline $\mathrm{H}$ & 0.6402790270 & 4.1038165444 & -2.4991292616 \\
\hline $\mathrm{C}$ & 0.6393068561 & 4.1282659434 & -1.4114346211 \\
\hline $\mathrm{C}$ & 0.6393070463 & 4.1282645332 & 1.4114373927 \\
\hline $\mathrm{C}$ & 1.8809490098 & 4.0896413307 & -0.7320583891 \\
\hline $\mathrm{C}$ & 1.8809490962 & 4.0896406893 & 0.7320610010 \\
\hline $\mathrm{H}$ & 0.6402793101 & 4.1038140179 & 2.4991319975 \\
\hline $\mathrm{C}$ & 3.0665810785 & 3.7852540652 & -1.4128391335 \\
\hline $\mathrm{C}$ & 3.0665812921 & 3.7852529552 & 1.4128413258 \\
\hline $\mathrm{H}$ & 3.0551720763 & 3.7583320228 & 2.5003194377 \\
\hline $\mathrm{H}$ & 3.0551716795 & 3.7583339617 & -2.5003172700 \\
\hline $\mathrm{C}$ & 4.1460355900 & 3.1802703516 & -0.7325138332 \\
\hline $\mathrm{C}$ & 4.1460357175 & 3.1802698665 & 0.7325154138 \\
\hline $\mathrm{C}$ & 5.0147208424 & 2.3119131668 & 1.4141536885 \\
\hline $\mathrm{C}$ & 5.0147204753 & 2.3119140270 & -1.4141528570 \\
\hline $\mathrm{H}$ & 4.9845386933 & 2.2903940334 & -2.5012292256 \\
\hline $\mathrm{H}$ & 4.9845393352 & 2.2903925264 & 2.5012300391 \\
\hline $\mathrm{C}$ & -0.5616597740 & 3.9295270261 & 0.7313088677 \\
\hline $\mathrm{C}$ & -0.5616598924 & 3.9295278201 & -0.7313061662 \\
\hline $\mathrm{C}$ & -1.7470274319 & 3.5299541054 & 1.4103018860 \\
\hline $\mathrm{C}$ & -1.7470278055 & 3.5299558001 & -1.4102992800 \\
\hline $\mathrm{H}$ & -1.7416720538 & 3.5134479436 & -2.4982584117 \\
\hline $\mathrm{H}$ & -1.7416712302 & 3.5134449360 & 2.4982609873 \\
\hline $\mathrm{C}$ & -2.8271801053 & 2.9963663629 & 0.7288111986 \\
\hline $\mathrm{C}$ & -2.8271803001 & 2.9963672836 & -0.7288088881 \\
\hline $\mathrm{H}$ & -6.1641785099 & 1.1942419803 & -2.4537547487 \\
\hline $\mathrm{C}$ & -5.8898149207 & 0.8246532645 & -1.4662189338 \\
\hline $\mathrm{C}$ & -5.8898150669 & 0.8246520790 & 1.4662193644 \\
\hline $\mathrm{C}$ & -4.8554389800 & 1.6091720520 & -0.7320000568 \\
\hline $\mathrm{C}$ & -4.8554388594 & 1.6091712542 & 0.7320012168 \\
\hline $\mathrm{H}$ & -6.1641792932 & 1.1942403847 & 2.4537551575 \\
\hline $\mathrm{C}$ & -3.9047810258 & 2.3259914127 & -1.4033641244 \\
\hline $\mathrm{C}$ & -3.9047806607 & 2.3259896939 & 1.4033658955 \\
\hline $\mathrm{H}$ & -3.9113178999 & 2.3395734226 & 2.4918412856 \\
\hline $\mathrm{H}$ & -3.9113184908 & 2.3395764104 & -2.4918394996 \\
\hline $\mathrm{H}$ & -1.7413116966 & -3.5135923151 & -2.4982604565 \\
\hline $\mathrm{C}$ & -1.7466665129 & -3.5301033840 & -1.4103013115 \\
\hline $\mathrm{C}$ & -1.7466665598 & -3.5301041374 & 1.4102993725 \\
\hline $\mathrm{C}$ & -2.8268689140 & -2.9966148260 & -0.7288108071 \\
\hline $\mathrm{C}$ & -2.8268689432 & -2.9966152367 & 0.7288091223 \\
\hline $\mathrm{H}$ & -1.7413117746 & -3.5135936964 & 2.4982585299 \\
\hline $\mathrm{C}$ & -3.9045357133 & -2.3263460266 & -1.4033652028 \\
\hline $\mathrm{C}$ & -3.9045358711 & -2.3263469183 & 1.4033638586 \\
\hline $\mathrm{H}$ & -3.9110673079 & -2.3399251448 & 2.4918394290 \\
\hline $\mathrm{H}$ & -3.9110670618 & -2.3399234797 & -2.4918407817 \\
\hline $\mathrm{C}$ & -4.8552743356 & -1.6096336486 & -0.7320008982 \\
\hline $\mathrm{C}$ & -4.8552744325 & -1.6096341653 & 0.7319999280 \\
\hline $\mathrm{C}$ & -6.7883267383 & -0.0003292794 & 0.6700360773 \\
\hline $\mathrm{C}$ & -6.7883268704 & -0.0003288108 & -0.6700366908 \\
\hline $\mathrm{C}$ & -5.8897334695 & -0.8252214055 & 1.4662180228 \\
\hline $\mathrm{C}$ & -5.8897330618 & -0.8252204227 & -1.4662187162 \\
\hline
\end{tabular}




\begin{tabular}{cccc}
$\mathrm{H}$ & -6.1640593659 & -1.1948372496 & -2.4537543179 \\
$\mathrm{H}$ & -6.1640602888 & -1.1948391802 & 2.4537531239 \\
$\mathrm{C}$ & -0.5612639284 & -3.9295712937 & 0.7313064793 \\
$\mathrm{C}$ & -0.5612639329 & -3.9295709296 & -0.7313085930 \\
$\mathrm{C}$ & 0.6397208279 & -4.1282052436 & 1.4114349495 \\
$\mathrm{C}$ & 0.6397208271 & -4.1282046038 & -1.4114371748 \\
$\mathrm{H}$ & 0.6406908851 & -4.1037559094 & -2.4991317348 \\
$\mathrm{H}$ & 0.6406908591 & -4.1037570763 & 2.4991295222 \\
$\mathrm{C}$ & 1.8813592111 & -4.0894697825 & 0.7320582527 \\
$\mathrm{C}$ & 1.8813591969 & -4.0894694843 & -0.7320604542 \\
$\mathrm{H}$ & 4.9847676671 & -2.2899187432 & -2.5012302748 \\
$\mathrm{C}$ & 5.0149543687 & -2.3114366958 & -1.4141540443 \\
$\mathrm{C}$ & 5.0149545200 & -2.3114374221 & 1.4141522916 \\
$\mathrm{C}$ & 4.1463591840 & -3.1798839770 & -0.7325153758 \\
$\mathrm{C}$ & 4.1463592277 & -3.1798843069 & 0.7325132959 \\
$\mathrm{H}$ & 4.9847679493 & -2.2899200302 & 2.5012285275 \\
$\mathrm{C}$ & 3.0669637403 & -3.7849729002 & -1.4128415484 \\
$\mathrm{C}$ & 3.0669637935 & -3.7849734684 & 1.4128393252 \\
$\mathrm{H}$ & 3.0555524468 & -3.7580559096 & -2.5003195473 \\
$\mathrm{H}$ & 3.0555527176 & -3.7580569317 & 2.5003173356 \\
$\mathrm{H}$ & 5.7489039068 & 0.0002752908 & 2.5014380657 \\
$\mathrm{C}$ & 5.7849686782 & 0.0002768856 & 1.4142463596 \\
$\mathrm{C}$ & 5.7849682625 & 0.0002776715 & -1.4142470055 \\
$\mathrm{C}$ & 5.5819076985 & 1.2154693583 & 0.7327851707 \\
$\mathrm{C}$ & 5.5820283982 & -1.2149349971 & 0.7327846373 \\
$\mathrm{C}$ & 5.5820282324 & -1.2149345850 & -0.7327858504 \\
$\mathrm{C}$ & 5.5819074936 & 1.2154697790 & -0.7327851524 \\
$\mathrm{H}$ & 5.7489031051 & 0.0002766395 & -2.5014386880 \\
\hline & & & \\
\hline
\end{tabular}


Table S22. Cartesian Coordinates in Angstrom for the optimized structure of Hückel-[13]cyclacene containing two Ladenburg benzenoids.

\begin{tabular}{|c|c|c|c|}
\hline Atom & $\mathrm{x}$-coordinate & y-coordinate & z-coordinate \\
\hline $\mathrm{H}$ & 0.0015138415 & 3.3488901209 & -2.4967430927 \\
\hline $\mathrm{C}$ & 0.0015159726 & 3.3735738542 & -1.4090151817 \\
\hline $\mathrm{C}$ & 0.0014922818 & 3.3740144307 & 1.4079321264 \\
\hline $\mathrm{H}$ & 0.0014718665 & 3.3496741663 & 2.4956679332 \\
\hline $\mathrm{C}$ & -1.2213234509 & 3.2760692155 & 0.7272448795 \\
\hline $\mathrm{C}$ & -1.2213110136 & 3.2758408981 & -0.7283175274 \\
\hline $\mathrm{C}$ & -2.4247716784 & 2.9684785340 & 1.4082413053 \\
\hline $\mathrm{C}$ & -2.4247471997 & 2.9680380753 & -1.4092396998 \\
\hline $\mathrm{H}$ & -2.4179110323 & 2.9450757831 & -2.4970492790 \\
\hline $\mathrm{H}$ & -2.4179566531 & 2.9458586813 & 2.4960582117 \\
\hline $\mathrm{C}$ & -3.5355594982 & 2.4910884086 & 0.7256588168 \\
\hline $\mathrm{C}$ & -3.5355474104 & 2.4908618090 & -0.7265281144 \\
\hline $\mathrm{H}$ & -6.7703248685 & 0.4259262111 & -2.4465545375 \\
\hline $\mathrm{C}$ & -6.4124077726 & 0.1063548767 & -1.4686350365 \\
\hline $\mathrm{C}$ & -6.4124352303 & 0.1068201553 & 1.4684646978 \\
\hline $\mathrm{C}$ & -5.5151291302 & 1.0471641769 & -0.7294787025 \\
\hline $\mathrm{C}$ & -5.5151427978 & 1.0473946240 & 0.7290268180 \\
\hline $\mathrm{H}$ & -6.7703701637 & 0.4267041568 & 2.4462756762 \\
\hline $\mathrm{C}$ & -4.6044064791 & 1.8143082218 & -1.4031665840 \\
\hline $\mathrm{C}$ & -4.6044303316 & 1.8147472722 & 1.4024902033 \\
\hline $\mathrm{H}$ & -4.5999302100 & 1.8134286069 & 2.4910104175 \\
\hline $\mathrm{H}$ & -4.5998901220 & 1.8126525978 & -2.4916863829 \\
\hline $\mathrm{H}$ & -1.2357749501 & -2.7036035147 & -2.4936815290 \\
\hline $\mathrm{C}$ & -1.2368578929 & -2.7161225121 & -1.4055511601 \\
\hline $\mathrm{C}$ & -1.2368814356 & -2.7156525963 & 1.4063713055 \\
\hline $\mathrm{C}$ & -2.4419236089 & -2.5649054480 & -0.7240312776 \\
\hline $\mathrm{C}$ & -2.4419352986 & -2.5646644350 & 0.7247798938 \\
\hline $\mathrm{H}$ & -1.2358184067 & -2.7027688185 & 2.4944973034 \\
\hline $\mathrm{C}$ & -3.6766939151 & -2.2983136787 & -1.4005611145 \\
\hline $\mathrm{C}$ & -3.6767159089 & -2.2978516680 & 1.4012030761 \\
\hline $\mathrm{H}$ & -3.6744447423 & -2.2996074762 & 2.4897442209 \\
\hline $\mathrm{H}$ & -3.6744084433 & -2.3004302565 & -2.4891013878 \\
\hline $\mathrm{C}$ & -4.8192606580 & -1.9529486887 & -0.7284418755 \\
\hline $\mathrm{C}$ & -4.8192727008 & -1.9527105164 & 0.7289533799 \\
\hline $\mathrm{C}$ & -7.1003371558 & -0.8987290430 & 0.6687106696 \\
\hline $\mathrm{C}$ & -7.1003203035 & -0.8989411582 & -0.6685746686 \\
\hline $\mathrm{C}$ & -6.0354014267 & -1.4904044594 & 1.4690358218 \\
\hline $\mathrm{C}$ & -6.0353733731 & -1.4908758785 & -1.4686927566 \\
\hline $\mathrm{H}$ & -6.2158170988 & -1.9358481918 & -2.4464033289 \\
\hline $\mathrm{H}$ & -6.2158579680 & -1.9350689606 & 2.4468837536 \\
\hline $\mathrm{C}$ & -0.0012184653 & -2.7702382978 & 0.7259914613 \\
\hline $\mathrm{C}$ & -0.0012062278 & -2.7704826666 & -0.7251314304 \\
\hline $\mathrm{C}$ & 1.2344813648 & -2.7167667388 & 1.4063938137 \\
\hline $\mathrm{C}$ & 1.2345052639 & -2.7172371876 & -1.4055317062 \\
\hline $\mathrm{H}$ & 1.2334516052 & -2.7047046540 & -2.4936614970 \\
\hline $\mathrm{H}$ & 1.2334106753 & -2.7038684629 & 2.4945192093 \\
\hline $\mathrm{C}$ & 2.4396830127 & -2.5668656666 & 0.7248205567 \\
\hline $\mathrm{C}$ & 2.4396958311 & -2.5671073744 & -0.7239890780 \\
\hline $\mathrm{H}$ & 6.2141690742 & -1.9414035157 & -2.4463024162 \\
\hline $\mathrm{C}$ & 6.0341030548 & -1.4962809734 & -1.4685897134 \\
\hline
\end{tabular}




\begin{tabular}{cccc}
$\mathrm{C}$ & 6.0340805739 & -1.4958079431 & 1.4691395482 \\
$\mathrm{C}$ & 4.8175809868 & -1.9572771862 & -0.7283592472 \\
$\mathrm{C}$ & 4.8175680857 & -1.9570378511 & 0.7290352830 \\
$\mathrm{H}$ & 6.2141256452 & -1.9406235760 & 2.4469955380 \\
$\mathrm{C}$ & 3.6747180534 & -2.3016323723 & -1.4004955008 \\
$\mathrm{C}$ & 3.6746920764 & -2.3011682440 & 1.4012627405 \\
$\mathrm{H}$ & 3.6724017651 & -2.3029320921 & 2.4898046521 \\
$\mathrm{H}$ & 3.6724514093 & -2.3037591035 & -2.4890366259 \\
$\mathrm{H}$ & 2.4205700613 & 2.9428742379 & -2.4970069945 \\
$\mathrm{C}$ & 2.4274123854 & 2.9658464314 & -1.4091980726 \\
$\mathrm{C}$ & 2.4273888078 & 2.9662861239 & 1.4082823140 \\
$\mathrm{C}$ & 3.5377715332 & 2.4876746833 & -0.7264676519 \\
$\mathrm{C}$ & 3.5377588357 & 2.4879008470 & 0.7257191010 \\
$\mathrm{H}$ & 2.4205296321 & 2.9436557682 & 2.4960983211 \\
$\mathrm{C}$ & 4.6060378309 & 1.8101686406 & -1.4030921735 \\
$\mathrm{C}$ & 4.6060138634 & 1.8106070410 & 1.4025727994 \\
$\mathrm{H}$ & 4.6014902081 & 1.8092887335 & 2.4910928039 \\
$\mathrm{H}$ & 4.6015344865 & 1.8085133369 & -2.4916119390 \\
$\mathrm{C}$ & 5.5160682028 & 1.0422233102 & -0.7293852084 \\
$\mathrm{C}$ & 5.5160569860 & 1.0424535372 & 0.7291213158 \\
$\mathrm{C}$ & 7.0995501250 & -0.9050699961 & 0.6688326994 \\
$\mathrm{C}$ & 7.0995552507 & -0.9052820302 & -0.6684535931 \\
$\mathrm{C}$ & 6.4125197976 & 0.1010858977 & 1.4685734182 \\
$\mathrm{C}$ & 6.4125425310 & 0.1006208905 & -1.4685245811 \\
$\mathrm{H}$ & 6.7707543056 & 0.4198732255 & -2.4464392870 \\
$\mathrm{H}$ & 6.7707166475 & 0.4206524431 & 2.4463912647 \\
$\mathrm{C}$ & 1.2242313891 & 3.2749742525 & 0.7272657804 \\
$\mathrm{C}$ & 1.2242435627 & 3.2747462288 & -0.7282971970 \\
\hline & & & \\
& & & \\
& & &
\end{tabular}


Table S23. Cartesian Coordinates in Angstrom for the optimized structure of Hückel-[14]cyclacene containing one Ladenburg benzenoid.

\begin{tabular}{|c|c|c|c|}
\hline Atom & $\mathrm{x}$-coordinate & y-coordinate & z-coordinate \\
\hline $\mathrm{H}$ & 0.0344348785 & 4.3304713813 & -2.4958884250 \\
\hline $\mathrm{C}$ & 0.0325929490 & 4.3494081952 & -1.4079902327 \\
\hline $\mathrm{C}$ & 0.0325929490 & 4.3494081952 & 1.4079902327 \\
\hline $\mathrm{C}$ & 1.2549283949 & 4.4494900851 & -0.7293023198 \\
\hline $\mathrm{C}$ & 1.2549283949 & 4.4494900851 & 0.7293023198 \\
\hline $\mathrm{H}$ & 0.0344348785 & 4.3304713813 & 2.4958884250 \\
\hline $\mathrm{C}$ & 2.4905783836 & 4.3228204506 & -1.4097227303 \\
\hline $\mathrm{C}$ & 2.4905783836 & 4.3228204506 & 1.4097227303 \\
\hline $\mathrm{H}$ & 2.4857843845 & 4.3036433499 & 2.4975175567 \\
\hline $\mathrm{H}$ & 2.4857843845 & 4.3036433499 & -2.4975175567 \\
\hline $\mathrm{C}$ & 3.6486823311 & 3.9212801075 & -0.7304871815 \\
\hline $\mathrm{C}$ & 3.6486823311 & 3.9212801075 & 0.7304871815 \\
\hline $\mathrm{C}$ & 4.6990532978 & 3.2585814418 & 1.4102901443 \\
\hline $\mathrm{C}$ & 4.6990532978 & 3.2585814418 & -1.4102901443 \\
\hline $\mathrm{H}$ & 4.6863758268 & 3.2443426346 & -2.4980147350 \\
\hline $\mathrm{H}$ & 4.6863758268 & 3.2443426346 & 2.4980147350 \\
\hline $\mathrm{C}$ & -1.1657583144 & 4.0479940186 & 0.7270072234 \\
\hline $\mathrm{C}$ & -1.1657583144 & 4.0479940186 & -0.7270072234 \\
\hline $\mathrm{C}$ & -2.3124243630 & 3.5861357575 & 1.4063447095 \\
\hline $\mathrm{C}$ & -2.3124243630 & 3.5861357575 & -1.4063447095 \\
\hline $\mathrm{H}$ & -2.3069914142 & 3.5713353725 & -2.4943734056 \\
\hline $\mathrm{H}$ & -2.3069914142 & 3.5713353725 & 2.4943734056 \\
\hline $\mathrm{C}$ & -3.3862882580 & 3.0116752168 & 0.7236260357 \\
\hline $\mathrm{C}$ & -3.3862882580 & 3.0116752168 & -0.7236260357 \\
\hline $\mathrm{H}$ & -6.7114331117 & 1.1986794932 & -2.4511904046 \\
\hline $\mathrm{C}$ & -6.4320041792 & 0.8205242015 & -1.4682503519 \\
\hline $\mathrm{C}$ & -6.4320041792 & 0.8205242015 & 1.4682503519 \\
\hline $\mathrm{C}$ & -5.3991814346 & 1.6055722429 & -0.7271571611 \\
\hline $\mathrm{C}$ & -5.3991814346 & 1.6055722429 & 0.7271571611 \\
\hline $\mathrm{H}$ & -6.7114331117 & 1.1986794932 & 2.4511904046 \\
\hline $\mathrm{C}$ & -4.4442029273 & 2.3253999282 & -1.3994905461 \\
\hline $\mathrm{C}$ & -4.4442029273 & 2.3253999282 & 1.3994905461 \\
\hline $\mathrm{H}$ & -4.4487551277 & 2.3360772426 & 2.4880067411 \\
\hline $\mathrm{H}$ & -4.4487551277 & 2.3360772426 & -2.4880067411 \\
\hline $\mathrm{H}$ & -2.3062221521 & -3.5717958814 & -2.4943737664 \\
\hline $\mathrm{C}$ & -2.3116521061 & -3.5865998318 & -1.4063451551 \\
\hline $\mathrm{C}$ & -2.3116521061 & -3.5865998318 & 1.4063451551 \\
\hline $\mathrm{C}$ & -3.3856355971 & -3.0123643329 & -0.7236259961 \\
\hline $\mathrm{C}$ & -3.3856355971 & -3.0123643329 & 0.7236259961 \\
\hline $\mathrm{H}$ & -2.3062221521 & -3.5717958814 & 2.4943737664 \\
\hline $\mathrm{C}$ & -4.4436974637 & -2.3263161792 & -1.3994901674 \\
\hline $\mathrm{C}$ & -4.4436974637 & -2.3263161792 & 1.3994901674 \\
\hline $\mathrm{H}$ & -4.4482465068 & -2.3369929414 & 2.4880063453 \\
\hline $\mathrm{H}$ & -4.4482465068 & -2.3369929414 & -2.4880063453 \\
\hline $\mathrm{C}$ & -5.3988362149 & -1.6067009717 & -0.7271573673 \\
\hline $\mathrm{C}$ & -5.3988362149 & -1.6067009717 & 0.7271573673 \\
\hline $\mathrm{C}$ & -7.3320009197 & -0.0007708379 & 0.6688435306 \\
\hline $\mathrm{C}$ & -7.3320009197 & -0.0007708379 & -0.6688435306 \\
\hline $\mathrm{C}$ & -6.4318296242 & -0.8218744553 & 1.4682501260 \\
\hline $\mathrm{C}$ & -6.4318296242 & -0.8218744553 & -1.4682501260 \\
\hline
\end{tabular}




\begin{tabular}{cccc}
$\mathrm{H}$ & -6.7111772275 & -1.2000895190 & -2.4511900880 \\
$\mathrm{H}$ & -6.7111772275 & -1.2000895190 & 2.4511900880 \\
$\mathrm{C}$ & -1.1648914370 & -4.0482229286 & 0.7270073890 \\
$\mathrm{C}$ & -1.1648914370 & -4.0482229286 & -0.7270073890 \\
$\mathrm{C}$ & 0.0335217294 & -4.3493908297 & 1.4079901894 \\
$\mathrm{C}$ & 0.0335217294 & -4.3493908297 & -1.4079901894 \\
$\mathrm{H}$ & 0.0353607390 & -4.3304515842 & -2.4958882813 \\
$\mathrm{H}$ & 0.0353607390 & -4.3304515842 & 2.4958882813 \\
$\mathrm{C}$ & 1.2558774538 & -4.4492250202 & 0.7293022814 \\
$\mathrm{C}$ & 1.2558774538 & -4.4492250202 & -0.7293022814 \\
$\mathrm{H}$ & 4.6870810226 & -3.2433819830 & -2.4980146935 \\
$\mathrm{C}$ & 4.6997580122 & -3.2576143056 & -1.4102899389 \\
$\mathrm{C}$ & 4.6997580122 & -3.2576143056 & 1.4102899389 \\
$\mathrm{C}$ & 3.6495227632 & -3.9205278463 & -0.7304870166 \\
$\mathrm{C}$ & 3.6495227632 & -3.9205278463 & 0.7304870166 \\
$\mathrm{H}$ & 4.6870810226 & -3.2433819830 & 2.4980146935 \\
$\mathrm{C}$ & 2.4915014571 & -4.3223054106 & -1.4097229046 \\
$\mathrm{C}$ & 2.4915014571 & -4.3223054106 & 1.4097229046 \\
$\mathrm{H}$ & 2.4867038176 & -4.3031297313 & -2.4975177130 \\
$\mathrm{H}$ & 2.4867038176 & -4.3031297313 & 2.4975177130 \\
$\mathrm{H}$ & 6.0394610132 & -1.2170238538 & 2.4978476656 \\
$\mathrm{C}$ & 6.0567839547 & -1.2218333105 & 1.4101078059 \\
$\mathrm{C}$ & 6.0567839547 & -1.2218333105 & -1.4101078059 \\
$\mathrm{C}$ & 5.5085560937 & -2.3320692766 & 0.7303496228 \\
$\mathrm{C}$ & 5.5085560937 & -2.3320692766 & -0.7303496228 \\
$\mathrm{H}$ & 6.0394610132 & -1.2170238538 & -2.4978476656 \\
$\mathrm{H}$ & 6.0392054446 & 1.2182829885 & -2.4978473772 \\
$\mathrm{C}$ & 6.0565215577 & 1.2230949463 & -1.4101072759 \\
$\mathrm{C}$ & 6.0565215577 & 1.2230949463 & 1.4101072759 \\
$\mathrm{C}$ & 6.2252947285 & 0.0006487272 & -0.7303857406 \\
$\mathrm{C}$ & 5.5080500968 & 2.3332112849 & -0.7303495738 \\
$\mathrm{C}$ & 5.5080500968 & 2.3332112849 & 0.7303495738 \\
$\mathrm{C}$ & 6.2252947285 & 0.0006487272 & 0.7303857406 \\
$\mathrm{H}$ & 6.0392054446 & 1.2182829885 & 2.4978473772 \\
\hline & & &
\end{tabular}


Table S24. Cartesian Coordinates in Angstrom for the optimized structure of Hückel-[14]cyclacene containing two Ladenburg benzenoids.

\begin{tabular}{|c|c|c|c|}
\hline Atom & $\mathrm{x}$-coordinate & y-coordinate & z-coordinate \\
\hline $\mathrm{H}$ & 0.0001282349 & 3.1446981597 & -2.4957424762 \\
\hline $\mathrm{C}$ & 0.0000763890 & 3.1618099555 & -1.4078204682 \\
\hline $\mathrm{C}$ & -0.0000576084 & 3.1617708581 & 1.4082842012 \\
\hline $\mathrm{H}$ & -0.0001097381 & 3.1446287514 & 2.4962057302 \\
\hline $\mathrm{C}$ & -1.2251031792 & 3.0966743590 & 0.7281248290 \\
\hline $\mathrm{C}$ & -1.2250339515 & 3.0966957001 & -0.7277793958 \\
\hline $\mathrm{C}$ & -2.4504107287 & 2.8914510056 & 1.4081147566 \\
\hline $\mathrm{C}$ & -2.4502763125 & 2.8914944583 & -1.4078933653 \\
\hline $\mathrm{H}$ & -2.4475628163 & 2.8770294390 & -2.4959604512 \\
\hline $\mathrm{H}$ & -2.4478036600 & 2.8769533388 & 2.4961815950 \\
\hline $\mathrm{C}$ & -3.6147614009 & 2.5645883428 & 0.7262715780 \\
\hline $\mathrm{C}$ & -3.6146925909 & 2.5646114030 & -0.7261721944 \\
\hline $\mathrm{H}$ & -7.2047342568 & 1.2158548105 & -2.4458537776 \\
\hline $\mathrm{C}$ & -6.9293261366 & 0.8210900283 & -1.4687420412 \\
\hline $\mathrm{C}$ & -6.9294626461 & 0.8210433779 & 1.4684718541 \\
\hline $\mathrm{C}$ & -5.8403667499 & 1.5333924244 & -0.7296605111 \\
\hline $\mathrm{C}$ & -5.8404359032 & 1.5333690668 & 0.7295145840 \\
\hline $\mathrm{H}$ & -7.2049575685 & 1.2157725909 & 2.4455735897 \\
\hline $\mathrm{C}$ & -4.7867330253 & 2.0890582435 & -1.4027048054 \\
\hline $\mathrm{C}$ & -4.7868662692 & 2.0890135944 & 1.4026770322 \\
\hline $\mathrm{H}$ & -4.7826156019 & 2.0851388195 & 2.4910911461 \\
\hline $\mathrm{H}$ & -4.7823779904 & 2.0852191801 & -2.4911186260 \\
\hline $\mathrm{H}$ & -2.4475791974 & -2.8769300793 & -2.4960550830 \\
\hline $\mathrm{C}$ & -2.4502933469 & -2.8914327857 & -1.4079884895 \\
\hline $\mathrm{C}$ & -2.4504287531 & -2.8914862585 & 1.4080196793 \\
\hline $\mathrm{C}$ & -3.6147077812 & -2.5645660724 & -0.7262565099 \\
\hline $\mathrm{C}$ & -3.6147771883 & -2.5645930413 & 0.7261872598 \\
\hline $\mathrm{H}$ & -2.4478221481 & -2.8770258038 & 2.4960870128 \\
\hline $\mathrm{C}$ & -4.7867449272 & -2.0889828346 & -1.4027735109 \\
\hline $\mathrm{C}$ & -4.7868795104 & -2.0890340380 & 1.4026083142 \\
\hline $\mathrm{H}$ & -4.7826292396 & -2.0851963870 & 2.4910225953 \\
\hline $\mathrm{H}$ & -4.7823893467 & -2.0851068779 & -2.4911871858 \\
\hline $\mathrm{C}$ & -5.8403756355 & -1.5333337256 & -0.7297108213 \\
\hline $\mathrm{C}$ & -5.8404453500 & -1.5333598981 & 0.7294642803 \\
\hline $\mathrm{C}$ & -7.8288791347 & 0.0000118377 & 0.6685856967 \\
\hline $\mathrm{C}$ & -7.8288159558 & 0.0000339069 & -0.6689633104 \\
\hline $\mathrm{C}$ & -6.9294679051 & -0.8210516174 & 1.4684448191 \\
\hline $\mathrm{C}$ & -6.9293306055 & -0.8210009696 & -1.4687690614 \\
\hline $\mathrm{H}$ & -7.2047408033 & -1.2157319144 & -2.4458939114 \\
\hline $\mathrm{H}$ & -7.2049655571 & -1.2158115038 & 2.4455333722 \\
\hline $\mathrm{H}$ & 0.0001096536 & -3.1446132616 & -2.4958456340 \\
\hline $\mathrm{C}$ & 0.0000574397 & -3.1617629293 & -1.4079242394 \\
\hline $\mathrm{C}$ & -0.0000770598 & -3.1618211517 & 1.4081804422 \\
\hline $\mathrm{C}$ & -1.2250525702 & -3.0966649231 & -0.7278811376 \\
\hline $\mathrm{C}$ & -1.2251221400 & -3.0966938381 & 0.7280231134 \\
\hline $\mathrm{H}$ & -0.0001292865 & -3.1447167675 & 2.4961025847 \\
\hline $\mathrm{C}$ & 1.2250332637 & -3.0967009957 & 0.7281400012 \\
\hline $\mathrm{C}$ & 1.2251025840 & -3.0966694973 & -0.7277642553 \\
\hline $\mathrm{C}$ & 2.4502755836 & -2.8915034838 & 1.4082553963 \\
\hline $\mathrm{C}$ & 2.4504099582 & -2.8914398494 & -1.4077527286 \\
\hline
\end{tabular}




\begin{tabular}{cccc}
$\mathrm{H}$ & 2.4478026004 & -2.8769339302 & -2.4958194415 \\
$\mathrm{H}$ & 2.4475619369 & -2.8770461155 & 2.4963226077 \\
$\mathrm{C}$ & 3.6146917303 & -2.5646149561 & 0.7265364224 \\
$\mathrm{C}$ & 3.6147602406 & -2.5645813917 & -0.7259074873 \\
$\mathrm{H}$ & 7.2049698452 & -1.2157772475 & -2.4451942315 \\
$\mathrm{C}$ & 6.9294667554 & -0.8210384545 & -1.4680985009 \\
$\mathrm{C}$ & 6.9293245468 & -0.8210995517 & 1.4691115801 \\
$\mathrm{C}$ & 5.8404355814 & -1.5333639408 & -0.7291448562 \\
$\mathrm{C}$ & 5.8403663036 & -1.5333970245 & 0.7300308216 \\
$\mathrm{H}$ & 7.2047266792 & -1.2158468277 & 2.4462317209 \\
$\mathrm{C}$ & 4.7868650956 & -2.0890020777 & -1.4023101982 \\
$\mathrm{C}$ & 4.7867320761 & -2.0890665157 & 1.4030720572 \\
$\mathrm{H}$ & 4.7823773516 & -2.0852340326 & 2.4914859006 \\
$\mathrm{H}$ & 4.7826136519 & -2.0851197821 & -2.4907243002 \\
$\mathrm{H}$ & 2.4478205685 & 2.8770065126 & -2.4957259034 \\
$\mathrm{C}$ & 2.4504277987 & 2.8914739941 & -1.4076586856 \\
$\mathrm{C}$ & 2.4502931498 & 2.8914386114 & 1.4083496160 \\
$\mathrm{C}$ & 3.6147763278 & 2.5645856235 & -0.7258246382 \\
$\mathrm{C}$ & 3.6147074691 & 2.5645676704 & 0.7266193221 \\
$\mathrm{H}$ & 2.4475792005 & 2.8769428729 & 2.4964163283 \\
$\mathrm{C}$ & 4.7868793780 & 2.0890243438 & -1.4022430966 \\
$\mathrm{C}$ & 4.7867455691 & 2.0889904155 & 1.4031383295 \\
$\mathrm{H}$ & 4.7823903784 & 2.0851202988 & 2.4915519929 \\
$\mathrm{H}$ & 4.7826288408 & 2.0851799831 & -2.4906573440 \\
$\mathrm{C}$ & 5.8404470586 & 1.5333574263 & -0.7290966972 \\
$\mathrm{C}$ & 5.8403776644 & 1.5333394257 & 0.7300780807 \\
$\mathrm{C}$ & 7.8288068805 & -0.0000336623 & 0.6693413954 \\
$\mathrm{C}$ & 7.8288850401 & -0.0000102354 & -0.6682077534 \\
$\mathrm{C}$ & 6.9293319158 & 0.8210134692 & 1.4691400722 \\
$\mathrm{C}$ & 6.9294737310 & 0.8210487787 & -1.4680739547 \\
$\mathrm{H}$ & 7.2049787760 & 1.2158149956 & -2.4451579564 \\
$\mathrm{H}$ & 7.2047309555 & 1.2157351322 & 2.4462707660 \\
$\mathrm{C}$ & 1.2250521712 & 3.0966669349 & 0.7282413045 \\
$\mathrm{C}$ & 1.2251213637 & 3.0966862264 & -0.7276630211 \\
\hline & & &
\end{tabular}


Table S25. Cartesian Coordinates in Angstrom for the optimized structure of Möbius-[9]cyclacene containing no Dewar benzenoids.

\begin{tabular}{|c|c|c|c|}
\hline Atom & $\mathrm{x}$-coordinate & y-coordinate & $\mathrm{z}$-coordinate \\
\hline $\mathrm{C}$ & 1.8689350429 & -2.8522887911 & 0.4191440084 \\
\hline $\mathrm{C}$ & -0.5326318996 & -2.9389833087 & 0.8774505817 \\
\hline $\mathrm{C}$ & -3.0191297463 & -2.0805755007 & -0.2074035661 \\
\hline $\mathrm{C}$ & -4.0592067214 & 0.0670626557 & -0.4861253480 \\
\hline $\mathrm{C}$ & -2.6657323413 & 1.7851721778 & -1.0792775019 \\
\hline $\mathrm{C}$ & -0.4481780361 & 1.8519572496 & -0.2758486429 \\
\hline $\mathrm{C}$ & 1.5880521718 & 1.9809302269 & 0.9576399896 \\
\hline $\mathrm{C}$ & 3.6124475653 & 1.3803663084 & -0.6066435200 \\
\hline $\mathrm{C}$ & 3.3793352765 & -0.9959242263 & -1.0896714075 \\
\hline $\mathrm{C}$ & 1.5811040107 & -2.6504742957 & -0.9904023344 \\
\hline $\mathrm{C}$ & -0.7982233608 & -3.0400407509 & -0.5503638805 \\
\hline $\mathrm{C}$ & -2.6469096830 & -1.7287059660 & 1.1562121321 \\
\hline $\mathrm{C}$ & -3.6951216849 & 0.4702492487 & 0.8788344243 \\
\hline $\mathrm{C}$ & -2.8160872800 & 2.6146621471 & 0.1513312637 \\
\hline $\mathrm{C}$ & -0.5136472831 & 3.3335155347 & -0.1307041428 \\
\hline $\mathrm{C}$ & 1.8958000467 & 2.9866342605 & -0.0841757188 \\
\hline $\mathrm{C}$ & 3.7206376238 & 0.9926954226 & 0.7949232794 \\
\hline $\mathrm{C}$ & 3.7414839385 & -1.3217831739 & 0.2762488624 \\
\hline $\mathrm{C}$ & 0.7911037532 & -3.0847520387 & 1.3142857188 \\
\hline $\mathrm{H}$ & 1.0003489240 & -3.0686718120 & 2.3818904880 \\
\hline $\mathrm{C}$ & -1.5333726853 & -2.3527275334 & 1.7170950748 \\
\hline $\mathrm{H}$ & -1.2665840275 & -2.1384734430 & 2.7500122554 \\
\hline $\mathrm{C}$ & -3.1732308743 & -0.4993861169 & 1.7137443244 \\
\hline $\mathrm{H}$ & -2.8555202807 & -0.2257294911 & 2.7183047762 \\
\hline $\mathrm{C}$ & -1.8241179232 & 3.7189487937 & 0.2269045713 \\
\hline $\mathrm{H}$ & -2.0673642442 & 4.6983154678 & 0.6295873676 \\
\hline $\mathrm{C}$ & 0.7570879301 & 3.9372247041 & -0.2117980068 \\
\hline $\mathrm{H}$ & 0.9080447330 & 5.0120534800 & -0.2640775828 \\
\hline $\mathrm{C}$ & 2.9754255721 & 2.6972870717 & -0.8705870073 \\
\hline $\mathrm{H}$ & 3.2129234434 & 3.2586064898 & -1.7707771423 \\
\hline $\mathrm{C}$ & 3.5995857079 & 0.3608150851 & -1.5444193873 \\
\hline $\mathrm{H}$ & 3.3851384440 & 0.5968554780 & -2.5850445591 \\
\hline $\mathrm{C}$ & 2.4541357655 & -1.8254306038 & -1.7485678448 \\
\hline $\mathrm{H}$ & 2.1814288223 & -1.5982236022 & -2.7772406669 \\
\hline $\mathrm{C}$ & 3.1006347727 & -2.3940721284 & 0.9291099975 \\
\hline $\mathrm{C}$ & 4.1988286845 & -0.2787278064 & 1.1340140736 \\
\hline $\mathrm{C}$ & 2.6300431603 & 1.4056688823 & 1.6480128420 \\
\hline $\mathrm{C}$ & 0.3834765668 & 1.2444017101 & 0.6083844934 \\
\hline $\mathrm{C}$ & -1.4040117085 & 1.3016194917 & -1.2118665603 \\
\hline $\mathrm{C}$ & -3.8263546180 & 1.0113780488 & -1.5172479725 \\
\hline $\mathrm{C}$ & -3.9945016198 & -1.2838322152 & -0.8717994070 \\
\hline $\mathrm{C}$ & -2.1390548157 & -2.8462362998 & -0.9869593961 \\
\hline $\mathrm{C}$ & 0.2808509415 & -2.9851966634 & -1.4508369094 \\
\hline $\mathrm{C}$ & -3.4479231065 & 1.9136949626 & 1.1476639510 \\
\hline $\mathrm{H}$ & -3.4778863933 & 2.2735637829 & 2.1736336617 \\
\hline $\mathrm{H}$ & 0.3540401990 & 0.1751070362 & 0.8013413132 \\
\hline $\mathrm{H}$ & 2.4213247947 & 0.7766144016 & 2.5101482551 \\
\hline $\mathrm{H}$ & 4.2804172804 & -0.5212251173 & 2.1912476937 \\
\hline $\mathrm{H}$ & 3.3060352132 & -2.5474889002 & 1.9862345282 \\
\hline $\mathrm{H}$ & 0.0667140468 & -2.9242922408 & -2.5160017085 \\
\hline
\end{tabular}




\begin{tabular}{llll}
$\mathrm{H}$ & -2.3711196057 & -2.9944572730 & -2.0394914864 \\
$\mathrm{H}$ & -4.2440658627 & -1.5351696231 & -1.9003430308 \\
$\mathrm{H}$ & -3.9632636184 & 0.7341778151 & -2.5590137666 \\
$\mathrm{H}$ & -1.1524307643 & 0.4630907612 & -1.8554572380 \\
\hline
\end{tabular}


Table S26. Cartesian Coordinates in Angstrom for the optimized structure of Möbius-[9]cyclacene containing one Dewar benzenoid.

\begin{tabular}{|c|c|c|c|}
\hline Atom & $\mathrm{x}$-coordinate & y-coordinate & z-coordinate \\
\hline $\mathrm{C}$ & 2.1734986524 & -1.9001260266 & -1.1444710451 \\
\hline $\mathrm{C}$ & 0.9216966455 & 1.5395036851 & 0.5000100885 \\
\hline $\mathrm{C}$ & -1.1525609423 & 1.7697896523 & -0.6859787671 \\
\hline $\mathrm{C}$ & -3.4932654984 & 1.4935554617 & -0.8573132072 \\
\hline $\mathrm{C}$ & -4.1533321029 & -0.6419227864 & -0.3125940988 \\
\hline $\mathrm{C}$ & -2.1999006568 & -2.2521289726 & 0.9525754717 \\
\hline $\mathrm{C}$ & 0.1035245149 & -2.9449979069 & 0.5098214231 \\
\hline $\mathrm{C}$ & 2.4599198060 & -2.3755768393 & 0.2050145634 \\
\hline $\mathrm{C}$ & 1.1651484120 & 2.9310536253 & 0.1206684869 \\
\hline $\mathrm{C}$ & -1.2756187994 & 3.0436133426 & 0.0338894108 \\
\hline $\mathrm{C}$ & -3.4134565022 & 2.0126618578 & 0.5204416732 \\
\hline $\mathrm{C}$ & -3.7216109378 & -0.3402690824 & 1.0508728964 \\
\hline $\mathrm{C}$ & -2.5007951876 & -2.3986865450 & -0.4608975343 \\
\hline $\mathrm{C}$ & -0.1236210599 & -2.7565815236 & -0.9093842638 \\
\hline $\mathrm{C}$ & 2.9644481095 & -0.8181523416 & -1.7180837433 \\
\hline $\mathrm{H}$ & 2.6574711134 & -0.4160714806 & -2.6812584237 \\
\hline $\mathrm{C}$ & 2.5073752270 & 3.1604081426 & -0.3765072204 \\
\hline $\mathrm{C}$ & -2.4919643402 & 3.1637947081 & 0.6979750300 \\
\hline $\mathrm{H}$ & -2.6664505689 & 3.9193025067 & 1.4590944108 \\
\hline $\mathrm{C}$ & -3.6879994778 & 1.0641047918 & 1.4869826756 \\
\hline $\mathrm{H}$ & -3.4927070866 & 1.2739732602 & 2.5363479558 \\
\hline $\mathrm{C}$ & -2.9460623625 & -1.2874346419 & 1.7123722389 \\
\hline $\mathrm{H}$ & -2.6240163456 & -1.0934993993 & 2.7336511845 \\
\hline $\mathrm{C}$ & -0.9751792773 & -2.7532920605 & 1.4136456752 \\
\hline $\mathrm{H}$ & -0.7531967195 & -2.7254918843 & 2.4783691352 \\
\hline $\mathrm{C}$ & 1.4383434086 & -2.9676712044 & 0.9620136913 \\
\hline $\mathrm{H}$ & 1.6337797314 & -3.1847548314 & 2.0102305693 \\
\hline $\mathrm{C}$ & 0.9495921009 & -2.2974338473 & -1.7151047735 \\
\hline $\mathrm{C}$ & -1.4717126627 & -2.7320589640 & -1.3549675141 \\
\hline $\mathrm{C}$ & -3.6919347767 & -1.7906685861 & -0.9566949952 \\
\hline $\mathrm{C}$ & -4.4890276442 & 0.4688177517 & -1.1658588564 \\
\hline $\mathrm{C}$ & -2.2780889220 & 1.3672573758 & -1.4783731037 \\
\hline $\mathrm{C}$ & -0.1652172930 & 0.9441749043 & -0.1945108872 \\
\hline $\mathrm{C}$ & 1.9098328914 & 0.8653281853 & 1.2506236929 \\
\hline $\mathrm{C}$ & 3.6007456630 & -1.8662446339 & 0.9071955459 \\
\hline $\mathrm{C}$ & 0.0038088811 & 3.7349017187 & 0.0969426299 \\
\hline $\mathrm{H}$ & 0.0634903594 & 4.8199490198 & 0.1023781371 \\
\hline $\mathrm{H}$ & -4.7210266244 & 0.2637896036 & -2.2089721115 \\
\hline $\mathrm{H}$ & -3.9432653449 & -1.9336816391 & -2.0052540785 \\
\hline $\mathrm{H}$ & -1.6740660040 & -2.6909793136 & -2.4232330936 \\
\hline $\mathrm{H}$ & 0.7353415868 & -2.0228984181 & -2.7464087760 \\
\hline $\mathrm{H}$ & 1.6860733167 & -0.0809931138 & 1.7318083367 \\
\hline $\mathrm{H}$ & -0.1836780631 & -0.1261810870 & -0.3681049491 \\
\hline $\mathrm{H}$ & -2.1543342994 & 0.7054707046 & -2.3309943074 \\
\hline $\mathrm{C}$ & 4.1812882892 & -0.7513982776 & 0.3808953955 \\
\hline $\mathrm{C}$ & 3.8956082760 & -0.2083011330 & -0.9132538514 \\
\hline $\mathrm{C}$ & 4.5575678528 & 0.5909686733 & 0.9323149160 \\
\hline $\mathrm{C}$ & 4.5300316967 & 1.2245006106 & -0.5346393024 \\
\hline $\mathrm{H}$ & 5.3542344987 & 0.7943135563 & 1.6505671815 \\
\hline $\mathrm{H}$ & 5.4294584587 & 1.5360895211 & -1.0734297186 \\
\hline
\end{tabular}




$\begin{array}{llll}\mathrm{C} & 3.2031400175 & 1.3216352046 & 1.0658758430 \\ \mathrm{C} & 3.4432975566 & 2.1961847767 & -0.0369953168 \\ \mathrm{H} & 3.7644607097 & -2.1669529089 & 1.9399401414 \\ \mathrm{H} & 2.6844138621 & 3.9598599176 & -1.0917923835\end{array}$


Table S27. Cartesian Coordinates in Angstrom for the optimized structure of Möbius-[10]cyclacene containing no Dewar benzenoids.

\begin{tabular}{|c|c|c|c|}
\hline Atom & $\mathrm{x}$-coordinate & y-coordinate & z-coordinate \\
\hline $\mathrm{C}$ & 0.7004191019 & -3.3759049052 & 0.6183228215 \\
\hline $\mathrm{C}$ & -1.6705858232 & -2.9135434734 & 1.0372684206 \\
\hline $\mathrm{C}$ & -3.9252348469 & -1.7081144469 & -0.2156738438 \\
\hline $\mathrm{C}$ & -4.3826171845 & 0.5852704147 & -0.6938083538 \\
\hline $\mathrm{C}$ & -2.6325664973 & 2.0527409641 & -1.1164583563 \\
\hline $\mathrm{C}$ & -0.4601809517 & 2.1359898307 & -0.1615947447 \\
\hline $\mathrm{C}$ & 1.6025177069 & 2.3150121126 & 1.0287901953 \\
\hline $\mathrm{C}$ & 3.7934367757 & 2.0131939088 & -0.4391833863 \\
\hline $\mathrm{C}$ & 4.0607994021 & -0.3330047314 & -1.0497324778 \\
\hline $\mathrm{C}$ & 2.6718856900 & -2.3467737121 & -1.1484620257 \\
\hline $\mathrm{C}$ & 0.4481248152 & -3.3275998604 & -0.8163977685 \\
\hline $\mathrm{C}$ & -1.9570711066 & -3.1389726126 & -0.3762245237 \\
\hline $\mathrm{C}$ & -3.5429798609 & -1.3339359070 & 1.1450352730 \\
\hline $\mathrm{C}$ & -4.1536764765 & 0.9933386755 & 0.7025633139 \\
\hline $\mathrm{C}$ & -2.9059245048 & 2.9593637133 & 0.0283280224 \\
\hline $\mathrm{C}$ & -0.5664213060 & 3.6171128547 & -0.0644146073 \\
\hline $\mathrm{C}$ & 1.8636057916 & 3.3923531814 & 0.0466730639 \\
\hline $\mathrm{C}$ & 3.8483197640 & 1.5634628633 & 0.9515755815 \\
\hline $\mathrm{C}$ & 4.4143411368 & -0.6640007027 & 0.3200596456 \\
\hline $\mathrm{C}$ & 2.9915023602 & -2.6220884503 & 0.2417360383 \\
\hline $\mathrm{C}$ & -0.3990396832 & -3.2366580122 & 1.5135573733 \\
\hline $\mathrm{H}$ & -0.1843982509 & -3.1288913293 & 2.5749195183 \\
\hline $\mathrm{C}$ & -2.5704162866 & -2.0865390960 & 1.7908808313 \\
\hline $\mathrm{H}$ & -2.2915061305 & -1.8335948345 & 2.8119009390 \\
\hline $\mathrm{C}$ & -3.8982611524 & -0.0063917043 & 1.6167430031 \\
\hline $\mathrm{H}$ & -3.6392696895 & 0.2548977737 & 2.6411022855 \\
\hline $\mathrm{C}$ & -1.8935136655 & 4.0271735691 & 0.1600657023 \\
\hline $\mathrm{H}$ & -2.1495584736 & 5.0285600007 & 0.4944988048 \\
\hline $\mathrm{C}$ & 0.6930353206 & 4.2575920046 & -0.1404405212 \\
\hline $\mathrm{H}$ & 0.7964159587 & 5.3204482015 & -0.3411939475 \\
\hline $\mathrm{C}$ & 3.0191197873 & 3.2470838371 & -0.6852738791 \\
\hline $\mathrm{H}$ & 3.2300636540 & 3.8677498809 & -1.5525868064 \\
\hline $\mathrm{C}$ & 4.0333279959 & 1.0692180288 & -1.4208091123 \\
\hline $\mathrm{H}$ & 3.8640138058 & 1.3267225399 & -2.4644914713 \\
\hline $\mathrm{C}$ & 3.3535235522 & -1.2807103164 & -1.8015742551 \\
\hline $\mathrm{H}$ & 3.0629002946 & -1.0330194104 & -2.8207447902 \\
\hline $\mathrm{C}$ & 1.4784552652 & -2.8975996446 & -1.6739737598 \\
\hline $\mathrm{H}$ & 1.2512076066 & -2.7533082886 & -2.7283522977 \\
\hline $\mathrm{C}$ & 2.0194378329 & -3.2288751278 & 1.0689410131 \\
\hline $\mathrm{C}$ & 4.0448351295 & -1.9073643758 & 0.8531123632 \\
\hline $\mathrm{C}$ & 4.5290578193 & 0.3910939248 & 1.2753345896 \\
\hline $\mathrm{C}$ & 2.6687679671 & 1.8006378328 & 1.7378822858 \\
\hline $\mathrm{C}$ & 0.4242873683 & 1.5590181565 & 0.7073896865 \\
\hline $\mathrm{C}$ & -1.3663965513 & 1.5362540399 & -1.0965349544 \\
\hline $\mathrm{C}$ & -3.7454781456 & 1.3320533776 & -1.6873926662 \\
\hline $\mathrm{C}$ & -4.6482017030 & -0.7752654924 & -1.0003941212 \\
\hline $\mathrm{C}$ & -3.2242613710 & -2.7303060997 & -0.8740357819 \\
\hline $\mathrm{C}$ & -0.9017482228 & -3.4283148391 & -1.2514864655 \\
\hline $\mathrm{C}$ & -3.7627933621 & 2.4028842413 & 0.9459506464 \\
\hline $\mathrm{H}$ & -3.9201142847 & 2.8429490097 & 1.9276149630 \\
\hline
\end{tabular}




\begin{tabular}{llll}
$\mathrm{H}$ & 0.3975054961 & 0.4971400888 & 0.9361100381 \\
$\mathrm{H}$ & 2.4890147900 & 1.1316299155 & 2.5757017807 \\
$\mathrm{H}$ & 4.5831788418 & 0.1017807507 & 2.3224031527 \\
$\mathrm{H}$ & 4.2536275104 & -2.1014957750 & 1.9028854779 \\
$\mathrm{H}$ & 2.2241331045 & -3.3043905985 & 2.1348245009 \\
$\mathrm{H}$ & -1.1060181395 & -3.4740807879 & -2.3191503578 \\
$\mathrm{H}$ & -3.4579093495 & -2.9297870158 & -1.9175274541 \\
$\mathrm{H}$ & -4.8527317319 & -1.0331886754 & -2.0372755410 \\
$\mathrm{H}$ & -3.6972880296 & 0.9530998619 & -2.7046637454 \\
$\mathrm{H}$ & -1.0998739536 & 0.6349132339 & -1.6412409469 \\
\hline
\end{tabular}


Table S28. Cartesian Coordinates in Angstrom for the optimized structure of Möbius-[10]cyclacene containing one Dewar benzenoid.

\begin{tabular}{|c|c|c|c|}
\hline Atom & $\mathrm{x}$-coordinate & $\mathrm{y}$-coordinate & z-coordinate \\
\hline $\mathrm{C}$ & -0.4330378349 & -2.8663036144 & 1.0010297666 \\
\hline $\mathrm{C}$ & -2.6906501683 & -1.8869371255 & 1.1631616974 \\
\hline $\mathrm{C}$ & -1.4949662281 & 1.6595892643 & -0.6122746558 \\
\hline $\mathrm{C}$ & 0.6058376760 & 1.9567194409 & 0.5251955726 \\
\hline $\mathrm{C}$ & 2.9328753058 & 2.1273622435 & 1.0469242986 \\
\hline $\mathrm{C}$ & 4.3140173660 & 0.9236952570 & -0.8863043378 \\
\hline $\mathrm{C}$ & 3.6772240851 & -1.4146841203 & -1.1433745131 \\
\hline $\mathrm{C}$ & 1.7247072016 & -2.8613299924 & -0.8519816192 \\
\hline $\mathrm{C}$ & -0.6644707890 & -3.1507587686 & -0.4041920588 \\
\hline $\mathrm{C}$ & -2.9830038661 & -2.4059602051 & -0.1710406552 \\
\hline $\mathrm{C}$ & -1.8054784805 & 3.0225358565 & -0.1774941881 \\
\hline $\mathrm{C}$ & 0.6174149681 & 3.3172755595 & -0.0134254926 \\
\hline $\mathrm{C}$ & 2.9949514281 & 2.8492631417 & -0.2275469109 \\
\hline $\mathrm{C}$ & 4.6468236154 & 0.6190819752 & 0.5073663399 \\
\hline $\mathrm{C}$ & 4.0653215143 & -1.6750376516 & 0.2394163619 \\
\hline $\mathrm{C}$ & 1.9839467405 & -2.8911897647 & 0.5819903313 \\
\hline $\mathrm{C}$ & -1.4828722485 & -2.2863309602 & 1.7588023767 \\
\hline $\mathrm{H}$ & -1.2639752383 & -1.9635665767 & 2.7748912677 \\
\hline $\mathrm{C}$ & -3.4852518663 & -0.7919116012 & 1.7065531380 \\
\hline $\mathrm{H}$ & -3.1846517914 & -0.3636242630 & 2.6603521907 \\
\hline $\mathrm{C}$ & -0.7033830704 & 3.8913769667 & -0.0902284551 \\
\hline $\mathrm{H}$ & -0.8413333087 & 4.9674517919 & -0.0203804211 \\
\hline $\mathrm{C}$ & 1.8718215516 & 3.7528855944 & -0.4608571609 \\
\hline $\mathrm{H}$ & 1.9699496879 & 4.6176657507 & -1.1114832970 \\
\hline $\mathrm{C}$ & 3.8144173974 & 2.2626323760 & -1.1872354204 \\
\hline $\mathrm{H}$ & 3.7797426456 & 2.6002775623 & -2.2202926301 \\
\hline $\mathrm{C}$ & 4.0417223736 & -0.1519239074 & -1.7308748701 \\
\hline $\mathrm{H}$ & 3.7336084312 & 0.0407809576 & -2.7564829418 \\
\hline $\mathrm{C}$ & 2.6610814813 & -2.1994599681 & -1.7012587077 \\
\hline $\mathrm{H}$ & 2.3894514973 & -2.0552952565 & -2.7448437363 \\
\hline $\mathrm{C}$ & 0.4371586616 & -3.2003512650 & -1.2920594152 \\
\hline $\mathrm{H}$ & 0.2378147042 & -3.2619067410 & -2.3599343523 \\
\hline $\mathrm{C}$ & 0.8990244284 & -2.9494766250 & 1.4740513412 \\
\hline $\mathrm{C}$ & 3.2822862363 & -2.5339512377 & 1.0284475362 \\
\hline $\mathrm{C}$ & 4.8771903194 & -0.7242345739 & 0.9078178678 \\
\hline $\mathrm{C}$ & 4.1021253593 & 1.4364292919 & 1.5071640899 \\
\hline $\mathrm{C}$ & 1.6893192711 & 1.6038264805 & 1.3655681021 \\
\hline $\mathrm{C}$ & -0.3497957473 & 1.1019841832 & -0.0247925034 \\
\hline $\mathrm{C}$ & -2.4854221127 & 0.9287726215 & -1.3318466769 \\
\hline $\mathrm{C}$ & -4.1087243319 & -1.8998966860 & -0.9008555773 \\
\hline $\mathrm{C}$ & -1.9883233289 & -3.0879273310 & -0.8809442973 \\
\hline $\mathrm{C}$ & -3.1516223798 & 3.1769369723 & 0.3486672156 \\
\hline $\mathrm{H}$ & -3.3455389740 & 3.9429797734 & 1.0954137410 \\
\hline $\mathrm{H}$ & 1.6065531476 & 0.7660821691 & 2.0528794909 \\
\hline $\mathrm{H}$ & 4.1458120284 & 1.1020971139 & 2.5406746307 \\
\hline $\mathrm{H}$ & 5.1209768117 & -0.9088777392 & 1.9516085959 \\
\hline $\mathrm{H}$ & 3.5086377914 & -2.6157182424 & 2.0892443546 \\
\hline $\mathrm{H}$ & 1.0911064549 & -2.8318079663 & 2.5386133942 \\
\hline $\mathrm{H}$ & -2.1854961826 & -3.3614209206 & -1.9154952010 \\
\hline $\mathrm{H}$ & -4.2667404860 & -2.2291344317 & -1.9256303623 \\
\hline
\end{tabular}




\begin{tabular}{llll}
$\mathrm{H}$ & -2.2221385430 & 0.0063701398 & -1.8414282791 \\
$\mathrm{H}$ & -0.2288140113 & 0.0237025274 & 0.0248299127 \\
$\mathrm{C}$ & -4.6985881049 & -0.7817567012 & -0.3997281754 \\
$\mathrm{C}$ & -4.4249632328 & -0.2182607244 & 0.8907683519 \\
$\mathrm{C}$ & -5.1138876260 & 0.5463090352 & -0.9590626950 \\
$\mathrm{C}$ & -5.1006574707 & 1.1843480127 & 0.5075535122 \\
$\mathrm{H}$ & -5.9193199767 & 0.7216210462 & -1.6749006604 \\
$\mathrm{H}$ & -6.0071653983 & 1.4752014092 & 1.0456071667 \\
$\mathrm{C}$ & -3.7814133314 & 1.3148965358 & -1.1037120156 \\
$\mathrm{C}$ & -4.0392643417 & 2.1797936171 & 0.0121148868 \\
\hline
\end{tabular}


Table S29. Cartesian Coordinates in Angstrom for the optimized structure of Möbius-[11]cyclacene containing no Dewar benzenoids.

\begin{tabular}{|c|c|c|c|}
\hline Atom & $\mathrm{x}$-coordinate & y-coordinate & z-coordinate \\
\hline $\mathrm{C}$ & 0.2026676427 & -3.7032994810 & 0.7180518056 \\
\hline $\mathrm{C}$ & -2.1377168640 & -3.0611448808 & 1.1029230597 \\
\hline $\mathrm{C}$ & -4.3407567187 & -1.7908633068 & -0.1794184802 \\
\hline $\mathrm{C}$ & -4.8818852576 & 0.5103975868 & -0.6370368852 \\
\hline $\mathrm{C}$ & -3.3507002428 & 2.2103695758 & -1.2035269924 \\
\hline $\mathrm{C}$ & -1.0617558696 & 2.4418894751 & -0.6075386531 \\
\hline $\mathrm{C}$ & 0.9404767439 & 2.5297983716 & 0.7083799221 \\
\hline $\mathrm{C}$ & 3.4352132915 & 3.0173051290 & -0.0903337874 \\
\hline $\mathrm{C}$ & 4.6148816165 & 0.9956475791 & -0.7442561541 \\
\hline $\mathrm{C}$ & 4.0730325290 & -1.3474123254 & -1.1700325725 \\
\hline $\mathrm{C}$ & 2.2893405217 & -3.0277989118 & -1.1021963798 \\
\hline $\mathrm{C}$ & -0.0434491827 & -3.7000914750 & -0.7224982186 \\
\hline $\mathrm{C}$ & -2.4293286925 & -3.3040035201 & -0.3068101233 \\
\hline $\mathrm{C}$ & -3.9664554305 & -1.4243791660 & 1.1816045099 \\
\hline $\mathrm{C}$ & -4.6203077638 & 0.8960852055 & 0.7518395720 \\
\hline $\mathrm{C}$ & -3.5449393524 & 2.9644504363 & 0.0522810981 \\
\hline $\mathrm{C}$ & -1.2815622866 & 3.8405646315 & -0.1620029089 \\
\hline $\mathrm{C}$ & 1.1600207468 & 3.8463688897 & 0.0680186650 \\
\hline $\mathrm{C}$ & 3.2723763575 & 2.3551215802 & 1.2055920568 \\
\hline $\mathrm{C}$ & 4.8401872494 & 0.6484931082 & 0.6486456760 \\
\hline $\mathrm{C}$ & 4.4224937062 & -1.6797379850 & 0.2006051096 \\
\hline $\mathrm{C}$ & 2.5780150065 & -3.2631418732 & 0.3080658386 \\
\hline $\mathrm{C}$ & 1.5295007273 & -3.6875903872 & 1.1648566901 \\
\hline $\mathrm{H}$ & 1.7275089533 & -3.7396834004 & 2.2334122505 \\
\hline $\mathrm{C}$ & -0.8864707406 & -3.4525987016 & 1.5966138561 \\
\hline $\mathrm{H}$ & -0.6737108485 & -3.3317649882 & 2.6568794041 \\
\hline $\mathrm{C}$ & -3.0064722332 & -2.1984175400 & 1.8371828523 \\
\hline $\mathrm{H}$ & -2.7322550420 & -1.9482308361 & 2.8600893142 \\
\hline $\mathrm{C}$ & -4.3269272133 & -0.1122527382 & 1.6573802566 \\
\hline $\mathrm{H}$ & -4.0606658868 & 0.1511116614 & 2.6793551268 \\
\hline $\mathrm{C}$ & -2.6016783930 & 4.0732655095 & 0.2451990474 \\
\hline $\mathrm{H}$ & -2.8744282501 & 4.9482632037 & 0.8292970541 \\
\hline $\mathrm{C}$ & -0.0786377643 & 4.5957163852 & -0.0603503083 \\
\hline $\mathrm{H}$ & -0.0846717133 & 5.6819416976 & -0.0241799330 \\
\hline $\mathrm{C}$ & 2.4508362328 & 4.0838982647 & -0.3591736912 \\
\hline $\mathrm{H}$ & 2.7068313994 & 4.9371082463 & -0.9820695824 \\
\hline $\mathrm{C}$ & 4.1919391700 & 2.3572755336 & -1.0361795068 \\
\hline $\mathrm{H}$ & 4.2100769536 & 2.7096876931 & -2.0651988658 \\
\hline $\mathrm{C}$ & 4.3925716217 & -0.0434631790 & -1.6538194628 \\
\hline $\mathrm{H}$ & 4.1400186620 & 0.2029986479 & -2.6833370596 \\
\hline $\mathrm{C}$ & 3.1359730931 & -2.1716599288 & -1.8365321910 \\
\hline $\mathrm{H}$ & 2.8766798733 & -1.9444014606 & -2.8685589435 \\
\hline $\mathrm{C}$ & 3.7666558331 & -2.7523405137 & 0.8464750676 \\
\hline $\mathrm{C}$ & 5.0218669335 & -0.6944019278 & 1.0120764088 \\
\hline $\mathrm{C}$ & 4.2492668285 & 1.4744528245 & 1.6510831251 \\
\hline $\mathrm{C}$ & 1.9089747655 & 2.0688475691 & 1.5647351880 \\
\hline $\mathrm{C}$ & -0.0953680065 & 1.7612104781 & 0.0773901899 \\
\hline $\mathrm{C}$ & -2.0449925075 & 1.9148811752 & -1.5074017134 \\
\hline $\mathrm{C}$ & -4.4042751414 & 1.3452433790 & -1.6536322698 \\
\hline $\mathrm{C}$ & -5.0441593485 & -0.8569493686 & -0.9678685357 \\
\hline
\end{tabular}




\begin{tabular}{llll}
$\mathrm{C}$ & -3.6580968693 & -2.8379447162 & -0.8274994753 \\
$\mathrm{C}$ & -1.3908996298 & -3.7067227292 & -1.1635522486 \\
$\mathrm{C}$ & 1.0264110155 & -3.4495713565 & -1.5976128851 \\
$\mathrm{C}$ & -4.2717560928 & 2.2954103347 & 1.0150667113 \\
$\mathrm{H}$ & -4.3214973312 & 2.6702761373 & 2.0348901373 \\
$\mathrm{H}$ & -0.0199385430 & 0.6770638285 & 0.0433260161 \\
$\mathrm{H}$ & 1.7242898762 & 1.2026373096 & 2.1950888550 \\
$\mathrm{H}$ & 4.1509304871 & 1.0484972826 & 2.6468061130 \\
$\mathrm{H}$ & 5.1733917893 & -0.9273950081 & 2.0635668484 \\
$\mathrm{H}$ & 3.9790873479 & -2.9264863291 & 1.8990355704 \\
$\mathrm{H}$ & 0.8195231754 & -3.3510273982 & -2.6613156408 \\
$\mathrm{H}$ & -1.5932997789 & -3.7834637356 & -2.2298154878 \\
$\mathrm{H}$ & -3.8936684777 & -3.0442245526 & -1.8693278651 \\
$\mathrm{H}$ & -5.2604133567 & -1.1141555191 & -2.0024361804 \\
$\mathrm{H}$ & -4.4047901762 & 0.9883286134 & -2.6804951063 \\
$\mathrm{H}$ & -1.7937750002 & 1.1359279021 & -2.2220157241 \\
\hline
\end{tabular}


Table S30. Cartesian Coordinates in Angstrom for the optimized structure of Möbius-[11]cyclacene containing one Dewar benzenoid.

\begin{tabular}{|c|c|c|c|}
\hline Atom & $\mathrm{x}$-coordinate & $\mathrm{y}$-coordinate & z-coordinate \\
\hline $\mathrm{C}$ & 3.1466190092 & 2.8519684572 & -0.4443775330 \\
\hline $\mathrm{C}$ & 0.5683592524 & 3.1650340019 & 0.7202385910 \\
\hline $\mathrm{C}$ & -1.8567228326 & 3.0589158153 & 0.3308101182 \\
\hline $\mathrm{C}$ & -4.0202237665 & 1.9589451791 & 0.0145387020 \\
\hline $\mathrm{C}$ & -5.1710596126 & -0.1598147332 & 0.2082815992 \\
\hline $\mathrm{C}$ & -4.3059154692 & -2.2076312113 & 1.0349763646 \\
\hline $\mathrm{C}$ & -1.9389864011 & -3.6860338490 & 0.4116803837 \\
\hline $\mathrm{C}$ & 0.4931533949 & -3.4291519837 & 0.3991638990 \\
\hline $\mathrm{C}$ & 2.8172620094 & -2.7009215407 & 0.1459823649 \\
\hline $\mathrm{C}$ & 2.9164889254 & 2.4351726860 & 0.9359710514 \\
\hline $\mathrm{C}$ & 0.7706427273 & 3.3759310951 & -0.7029430558 \\
\hline $\mathrm{C}$ & -1.6149428372 & 2.9865936603 & -1.1058330055 \\
\hline $\mathrm{C}$ & -3.6525633650 & 1.6455202439 & -1.3625516299 \\
\hline $\mathrm{C}$ & -4.7109093838 & -0.5446383155 & -1.1237844752 \\
\hline $\mathrm{C}$ & -4.0640733974 & -2.7432589316 & -0.3058446796 \\
\hline $\mathrm{C}$ & -1.9852232942 & -2.5889106454 & 1.3803792161 \\
\hline $\mathrm{C}$ & 0.1935978400 & -2.0084374568 & 0.5394463707 \\
\hline $\mathrm{C}$ & 2.2970521061 & -1.5275469427 & -0.5493116107 \\
\hline $\mathrm{C}$ & 4.3236120963 & 2.4180501668 & -1.1329182973 \\
\hline $\mathrm{H}$ & 4.4411039471 & 2.6570744743 & -2.1877118161 \\
\hline $\mathrm{C}$ & 2.0881676840 & 3.3757275580 & -1.1943295404 \\
\hline $\mathrm{H}$ & 2.2531539366 & 3.5846310733 & -2.2494059507 \\
\hline $\mathrm{C}$ & -0.3394583422 & 3.3283559507 & -1.5766294867 \\
\hline $\mathrm{H}$ & -0.1570382541 & 3.3536993680 & -2.6489897103 \\
\hline $\mathrm{C}$ & -4.1667561244 & 0.4502035990 & -1.9483662834 \\
\hline $\mathrm{H}$ & -3.8286697477 & 0.1825423160 & -2.9474306425 \\
\hline $\mathrm{C}$ & -4.4461037822 & -1.9357648874 & -1.3818901642 \\
\hline $\mathrm{H}$ & -4.1737449051 & -2.2328844233 & -2.3924180317 \\
\hline $\mathrm{C}$ & -3.0612822371 & -3.7812125529 & -0.4258063827 \\
\hline $\mathrm{H}$ & -3.0208557873 & -4.3946179694 & -1.3226197743 \\
\hline $\mathrm{C}$ & -0.6378114715 & -4.2629708085 & 0.2211410837 \\
\hline $\mathrm{H}$ & -0.5254471791 & -5.2574790992 & -0.2030077848 \\
\hline $\mathrm{C}$ & 1.8840389287 & -3.7315062281 & 0.3989670414 \\
\hline $\mathrm{H}$ & 2.2263209010 & -4.7311426266 & 0.6559340876 \\
\hline $\mathrm{C}$ & 4.1412596775 & -2.5514987318 & 0.7042710191 \\
\hline $\mathrm{H}$ & 4.4371862777 & -3.1693579238 & 1.5485994603 \\
\hline $\mathrm{C}$ & 3.1920477184 & -0.7263695862 & -1.3212830023 \\
\hline $\mathrm{C}$ & 1.0079169342 & -1.1244428518 & -0.1692997299 \\
\hline $\mathrm{C}$ & -0.9388819656 & -1.6847758236 & 1.3263877030 \\
\hline $\mathrm{C}$ & -3.2525146988 & -2.2428051202 & 1.9391215532 \\
\hline $\mathrm{C}$ & -5.2496457975 & -1.1484867686 & 1.2110220623 \\
\hline $\mathrm{C}$ & -5.0154537458 & 1.1716044559 & 0.6430302361 \\
\hline $\mathrm{C}$ & -3.1589576301 & 2.7431860681 & 0.7964179816 \\
\hline $\mathrm{C}$ & -0.7635850415 & 3.1896490692 & 1.2032555635 \\
\hline $\mathrm{C}$ & 1.6701178543 & 2.7477022069 & 1.5067431750 \\
\hline $\mathrm{C}$ & 3.8517143517 & 1.5321414068 & 1.5864672760 \\
\hline $\mathrm{C}$ & -2.5751525356 & 2.3413499526 & -1.9307717697 \\
\hline $\mathrm{H}$ & -2.3240318894 & 2.1719783905 & -2.9756932554 \\
\hline $\mathrm{H}$ & -5.4842876316 & -0.8349883176 & 2.2258761417 \\
\hline $\mathrm{H}$ & -3.2962333311 & -1.6310581147 & 2.8363350564 \\
\hline
\end{tabular}




\begin{tabular}{lccc}
$\mathrm{H}$ & -1.0432933396 & -0.6928692223 & 1.7578401631 \\
$\mathrm{H}$ & 0.6783143283 & -0.1072107313 & -0.3651177613 \\
$\mathrm{H}$ & 2.8027157580 & 0.0415108419 & -1.9842326066 \\
$\mathrm{H}$ & 3.6109974485 & 1.1730041987 & 2.5848485737 \\
$\mathrm{H}$ & 1.4929510437 & 2.4960808549 & 2.5507896231 \\
$\mathrm{H}$ & -0.9382534211 & 3.1250317083 & 2.2753329448 \\
$\mathrm{H}$ & -3.3831100336 & 2.8701503053 & 1.8534090743 \\
$\mathrm{H}$ & -5.3034019662 & 1.4190933255 & 1.6622782393 \\
$\mathrm{C}$ & 4.8679134109 & -1.4764438735 & 0.2425517349 \\
$\mathrm{C}$ & 4.5156220295 & -0.8203936524 & -0.9784759994 \\
$\mathrm{C}$ & 5.7646288021 & -0.2893463829 & 0.6146803407 \\
$\mathrm{C}$ & 5.7029898118 & 0.1654626870 & -0.9186324374 \\
$\mathrm{H}$ & 6.6951417822 & -0.3812083078 & 1.1813779193 \\
$\mathrm{H}$ & 6.5405403203 & 0.0453536032 & -1.6086124150 \\
$\mathrm{C}$ & 4.8795230632 & 1.0228034246 & 0.8338487389 \\
$\mathrm{C}$ & 5.0715155694 & 1.4638950630 & -0.5145372556 \\
\hline
\end{tabular}


Table S31. Cartesian Coordinates in Angstrom for the optimized structure of Möbius-[12]cyclacene containing no Dewar benzenoids.

\begin{tabular}{|c|c|c|c|}
\hline Atom & $\mathrm{x}$-coordinate & y-coordinate & z-coordinate \\
\hline $\mathrm{C}$ & 3.8023324642 & -2.2726323196 & -1.2394570619 \\
\hline $\mathrm{C}$ & 4.9936132650 & -0.1410737578 & -1.0140285224 \\
\hline $\mathrm{C}$ & 4.7552196445 & 1.7212625157 & 1.0206778151 \\
\hline $\mathrm{C}$ & 2.6078317326 & 2.7192258971 & 1.2032167243 \\
\hline $\mathrm{C}$ & 0.4245317859 & 2.7253294613 & 0.2136934601 \\
\hline $\mathrm{C}$ & -1.6525522151 & 2.8309193731 & -0.9937777525 \\
\hline $\mathrm{C}$ & -3.9914743834 & 2.3773889195 & -1.2183936790 \\
\hline $\mathrm{C}$ & -5.0974355415 & 0.8410060053 & 0.7862667503 \\
\hline $\mathrm{C}$ & -4.4722743695 & -1.4879868738 & 1.2022916959 \\
\hline $\mathrm{C}$ & -2.7021279778 & -3.1885291464 & 1.1597976212 \\
\hline $\mathrm{C}$ & -0.3952993804 & -3.9724854419 & 0.8130647506 \\
\hline $\mathrm{C}$ & 1.7516733144 & -3.6134223489 & -1.0380209579 \\
\hline $\mathrm{C}$ & 4.1488285684 & -2.6097904044 & 0.1370831910 \\
\hline $\mathrm{C}$ & 5.3390951880 & -0.5034480693 & 0.3568506230 \\
\hline $\mathrm{C}$ & 4.7095686647 & 2.1980328401 & -0.3654035321 \\
\hline $\mathrm{C}$ & 2.9065970382 & 3.7391098843 & 0.1779880368 \\
\hline $\mathrm{C}$ & 0.5039551492 & 4.1988383248 & 0.0908812315 \\
\hline $\mathrm{C}$ & -1.9295378296 & 4.0204089592 & -0.1651190300 \\
\hline $\mathrm{C}$ & -4.1035643211 & 2.9642037744 & 0.1172595565 \\
\hline $\mathrm{C}$ & -5.3385736236 & 0.4956335816 & -0.6042069486 \\
\hline $\mathrm{C}$ & -4.8099463018 & -1.8228070275 & -0.1700971746 \\
\hline $\mathrm{C}$ & -2.9883460992 & -3.4396599436 & -0.2483096539 \\
\hline $\mathrm{C}$ & -0.6379039440 & -4.0231363085 & -0.6285597673 \\
\hline $\mathrm{C}$ & 2.0204961756 & -3.7963614125 & 0.3870704380 \\
\hline $\mathrm{C}$ & 2.7121237661 & -2.9298970052 & -1.8298944290 \\
\hline $\mathrm{H}$ & 2.4653715004 & -2.7170018656 & -2.8680603228 \\
\hline $\mathrm{C}$ & 4.3870881180 & -1.1059855118 & -1.8210507867 \\
\hline $\mathrm{H}$ & 4.0998720500 & -0.8400022508 & -2.8364698878 \\
\hline $\mathrm{C}$ & 4.9548761250 & 1.2627676356 & -1.3588859163 \\
\hline $\mathrm{H}$ & 4.8037439641 & 1.5357941289 & -2.4013113845 \\
\hline $\mathrm{C}$ & 4.0201547300 & 3.4676794043 & -0.5989995045 \\
\hline $\mathrm{H}$ & 4.2218642235 & 4.0335757244 & -1.5054060371 \\
\hline $\mathrm{C}$ & -0.7542705228 & 4.8418818154 & 0.0676230247 \\
\hline $\mathrm{H}$ & -0.8478380514 & 5.9100854192 & 0.2457534292 \\
\hline $\mathrm{C}$ & -3.1918039464 & 4.0797365064 & 0.3949391742 \\
\hline $\mathrm{H}$ & -3.4373995725 & 4.8139106221 & 1.1583000781 \\
\hline $\mathrm{C}$ & -4.7478411856 & 2.2154062415 & 1.0856229807 \\
\hline $\mathrm{H}$ & -4.7220296537 & 2.5379761600 & 2.1243733665 \\
\hline $\mathrm{C}$ & -4.8149596347 & -0.1939414193 & 1.6864705581 \\
\hline $\mathrm{H}$ & -4.5619091150 & 0.0583143786 & 2.7144726810 \\
\hline $\mathrm{C}$ & -3.5350544672 & -2.3087973781 & 1.8763117440 \\
\hline $\mathrm{H}$ & -3.2817272961 & -2.0765497499 & 2.9087405391 \\
\hline $\mathrm{C}$ & -1.4538369477 & -3.6386784874 & 1.6714984433 \\
\hline $\mathrm{H}$ & -1.2489100451 & -3.5191554523 & 2.7334645609 \\
\hline $\mathrm{C}$ & 0.9483293732 & -4.0478880466 & 1.2601982031 \\
\hline $\mathrm{H}$ & 1.1436443562 & -4.0858019962 & 2.3298187361 \\
\hline $\mathrm{C}$ & 0.4651346750 & -3.9100714032 & -1.5132639193 \\
\hline $\mathrm{C}$ & -1.9579952076 & -3.9379768386 & -1.0859539890 \\
\hline $\mathrm{C}$ & -4.1507230690 & -2.8969613936 & -0.8085778535 \\
\hline $\mathrm{C}$ & -5.4280868265 & -0.8512503348 & -0.9816270498 \\
\hline
\end{tabular}




\begin{tabular}{cccc}
$\mathrm{C}$ & -4.8946074502 & 1.3961827552 & -1.6145580271 \\
$\mathrm{C}$ & -2.6759219269 & 2.3288089885 & -1.7752372723 \\
$\mathrm{C}$ & -0.4970421027 & 2.0988145104 & -0.5942348681 \\
$\mathrm{C}$ & 1.3622562100 & 2.1359307718 & 1.1058645503 \\
$\mathrm{C}$ & 3.7077020226 & 2.0935587883 & 1.8590746604 \\
$\mathrm{C}$ & 5.4411201266 & 0.5141492263 & 1.3215409556 \\
$\mathrm{C}$ & 5.1003869737 & -1.8189469585 & 0.8100531501 \\
$\mathrm{C}$ & 3.3044523626 & -3.4649469642 & 0.8718340692 \\
$\mathrm{C}$ & 1.8223321484 & 4.6815343701 & -0.0472503356 \\
$\mathrm{H}$ & 2.0196329712 & 5.6840491504 & -0.4180383179 \\
$\mathrm{H}$ & -2.4698273223 & 1.5707066364 & -2.5266507342 \\
$\mathrm{H}$ & -4.8462407946 & 1.0205730717 & -2.6341098306 \\
$\mathrm{H}$ & -5.5890743899 & -1.0870834171 & -2.0311332504 \\
$\mathrm{H}$ & -4.3627159361 & -3.0832806834 & -1.8592155435 \\
$\mathrm{H}$ & -2.1544514831 & -4.0208691887 & -2.1528431700 \\
$\mathrm{H}$ & 0.2656380261 & -3.8380288397 & -2.5804800321 \\
$\mathrm{H}$ & 3.5272139415 & -3.6373628382 & 1.9227085081 \\
$\mathrm{H}$ & 5.3350302897 & -2.0586835134 & 1.8448509017 \\
$\mathrm{H}$ & 5.5517608933 & 0.2280272921 & 2.3650252743 \\
$\mathrm{H}$ & 3.5530469863 & 1.5630590316 & 2.7951679142 \\
$\mathrm{H}$ & 1.1496191164 & 1.1834715789 & 1.5836877759 \\
$\mathrm{H}$ & -0.4517083568 & 1.0289310145 & -0.7798798627 \\
\hline
\end{tabular}


Table S32. Cartesian Coordinates in Angstrom for the optimized structure of Möbius-[12]cyclacene containing one Dewar benzenoid.

\begin{tabular}{|c|c|c|c|}
\hline Atom & $\mathrm{x}$-coordinate & $\mathrm{y}$-coordinate & z-coordinate \\
\hline $\mathrm{C}$ & 2.9025463653 & -3.2498901220 & -0.2998972176 \\
\hline $\mathrm{C}$ & 0.4972019166 & -3.6709338530 & -0.1127672725 \\
\hline $\mathrm{C}$ & -1.8317740273 & -2.5881325445 & 0.9766182791 \\
\hline $\mathrm{C}$ & -4.2147222394 & -2.3207629098 & 1.0506114995 \\
\hline $\mathrm{C}$ & -5.6334471834 & -0.5058805320 & 0.4497874443 \\
\hline $\mathrm{C}$ & -5.0326059043 & 1.8084540801 & 0.1774990425 \\
\hline $\mathrm{C}$ & -3.0677085691 & 3.2268302492 & 0.4422488498 \\
\hline $\mathrm{C}$ & -0.4423321405 & 3.6899400767 & -0.5632874712 \\
\hline $\mathrm{C}$ & 1.9613246453 & 3.3744239423 & -0.2019420787 \\
\hline $\mathrm{C}$ & 4.1580954196 & 2.2995942252 & -0.1154468982 \\
\hline $\mathrm{C}$ & 2.5745717490 & -1.9693570578 & -0.9177437133 \\
\hline $\mathrm{C}$ & 0.3670464647 & -2.2287346309 & 0.0434611317 \\
\hline $\mathrm{C}$ & -1.9470894622 & -3.7246130434 & 0.0606287517 \\
\hline $\mathrm{C}$ & -4.2054728330 & -2.8704504911 & -0.3064526555 \\
\hline $\mathrm{C}$ & -5.3014987502 & -0.7925503342 & -0.9448097574 \\
\hline $\mathrm{C}$ & -4.6569356364 & 1.5474110784 & -1.2088236766 \\
\hline $\mathrm{C}$ & -2.7943578770 & 3.1262475119 & -0.9889007222 \\
\hline $\mathrm{C}$ & -0.6692276764 & 3.5262842020 & 0.8690067709 \\
\hline $\mathrm{C}$ & 1.7095788904 & 2.9742494283 & 1.1716433852 \\
\hline $\mathrm{C}$ & 3.8515607723 & 1.7476961652 & 1.2013939245 \\
\hline $\mathrm{C}$ & 4.2152314977 & -3.3453706761 & 0.2972998006 \\
\hline $\mathrm{H}$ & 4.3878737588 & -4.0758542022 & 1.0839287148 \\
\hline $\mathrm{C}$ & 1.8319022733 & -4.1522172635 & -0.1229953251 \\
\hline $\mathrm{H}$ & 2.0301051472 & -5.1991476461 & 0.0950987397 \\
\hline $\mathrm{C}$ & -0.7218971993 & -4.3874401910 & -0.2381687029 \\
\hline $\mathrm{H}$ & -0.7235599052 & -5.3878062520 & -0.6640077748 \\
\hline $\mathrm{C}$ & -3.1908901826 & -3.8508349739 & -0.5919612337 \\
\hline $\mathrm{H}$ & -3.2790971105 & -4.4899584326 & -1.4670964734 \\
\hline $\mathrm{C}$ & -5.0146464805 & 0.2910354303 & -1.7867867037 \\
\hline $\mathrm{H}$ & -4.7217207638 & 0.0969136150 & -2.8165502892 \\
\hline $\mathrm{C}$ & -3.6864206109 & 2.3718972776 & -1.8001014088 \\
\hline $\mathrm{H}$ & -3.4233908016 & 2.2149303999 & -2.8441095111 \\
\hline $\mathrm{C}$ & -1.5380863402 & 3.5431290199 & -1.4523819524 \\
\hline $\mathrm{H}$ & -1.3382106228 & 3.5397248660 & -2.5219013369 \\
\hline $\mathrm{C}$ & 0.8799335076 & 3.7662933438 & -1.0204378993 \\
\hline $\mathrm{H}$ & 1.0662276169 & 3.9506942751 & -2.0764475187 \\
\hline $\mathrm{C}$ & 3.2406076707 & 3.1476360124 & -0.7401481274 \\
\hline $\mathrm{H}$ & 3.4464548111 & 3.4790333486 & -1.7558012570 \\
\hline $\mathrm{C}$ & 5.2165925246 & 1.7351312024 & -0.8995331047 \\
\hline $\mathrm{C}$ & 4.5980665964 & 0.6025240230 & 1.6984709546 \\
\hline $\mathrm{C}$ & 2.6946360194 & 2.2121607308 & 1.8474972226 \\
\hline $\mathrm{C}$ & 0.4181200218 & 3.2115320707 & 1.7012315374 \\
\hline $\mathrm{C}$ & -2.0112871721 & 3.4916809742 & 1.3254955325 \\
\hline $\mathrm{C}$ & -4.3170937186 & 2.7642864846 & 0.9198660900 \\
\hline $\mathrm{C}$ & -5.7824054743 & 0.8369640937 & 0.8736979869 \\
\hline $\mathrm{C}$ & -5.2672541590 & -1.4352416768 & 1.4321935703 \\
\hline $\mathrm{C}$ & -2.9961603834 & -2.2105654138 & 1.7018303376 \\
\hline $\mathrm{C}$ & -0.7175056204 & -1.7772340718 & 0.8309342748 \\
\hline $\mathrm{C}$ & 1.3257882722 & -1.4283007745 & -0.5872431695 \\
\hline $\mathrm{C}$ & 3.6087904527 & -1.2189453000 & -1.5631909636 \\
\hline
\end{tabular}




\begin{tabular}{lccc}
$\mathrm{C}$ & -4.8847316046 & -2.1287076625 & -1.2815894316 \\
$\mathrm{H}$ & -4.7750559719 & -2.3981888239 & -2.3296676009 \\
$\mathrm{H}$ & -4.5454889299 & 2.8793305909 & 1.9771522984 \\
$\mathrm{H}$ & -2.2010925228 & 3.4537129497 & 2.3960786014 \\
$\mathrm{H}$ & 0.2293493308 & 2.9908710293 & 2.7499643316 \\
$\mathrm{H}$ & 2.4657703019 & 1.8440128589 & 2.8458476653 \\
$\mathrm{H}$ & 4.3017388487 & 0.1658061683 & 2.6497496195 \\
$\mathrm{H}$ & 1.1417217909 & -0.3673362203 & -0.7389818477 \\
$\mathrm{H}$ & -0.7056686298 & -0.7759416996 & 1.2539035813 \\
$\mathrm{H}$ & -2.9072112787 & -1.5704973986 & 2.5757823974 \\
$\mathrm{H}$ & -5.3576863030 & -1.1533214335 & 2.4785209062 \\
$\mathrm{H}$ & -6.0247688799 & 1.0227713176 & 1.9176330405 \\
$\mathrm{C}$ & 5.7723785959 & 0.5868902610 & -0.4323592230 \\
$\mathrm{C}$ & 5.5058962923 & 0.0191699925 & 0.8556397319 \\
$\mathrm{C}$ & 6.2006667121 & -0.7350245997 & -0.9993994447 \\
$\mathrm{C}$ & 6.1694732815 & -1.3742570626 & 0.4690131355 \\
$\mathrm{H}$ & 7.0252166131 & -0.8908045504 & -1.6979088339 \\
$\mathrm{H}$ & 7.0664952169 & -1.6830289060 & 1.0123905472 \\
$\mathrm{C}$ & 4.8866008111 & -1.5216385964 & -1.1849288799 \\
$\mathrm{C}$ & 5.1025245130 & -2.3512120766 & -0.0394592620 \\
$\mathrm{H}$ & 5.3765487831 & 2.0941306702 & -1.9138238630 \\
$\mathrm{H}$ & 3.3563881015 & -0.3461335043 & -2.1602519757 \\
\hline
\end{tabular}


Table S33. Cartesian Coordinates in Angstrom for the optimized structure of Möbius-[13]cyclacene containing no Dewar benzenoids.

\begin{tabular}{|c|c|c|c|}
\hline Atom & $\mathrm{X}$-coordinate & $\mathrm{y}$-coordinate & $\mathrm{z}$-coordinate \\
\hline $\mathrm{C}$ & 4.3345210502 & -2.3994211264 & -1.2273269929 \\
\hline $\mathrm{C}$ & 5.4877007120 & -0.2543867494 & -0.9711687671 \\
\hline $\mathrm{C}$ & 5.2204488843 & 1.6335562892 & 1.0451669251 \\
\hline $\mathrm{C}$ & 3.1375112604 & 2.7823223935 & 1.2101422214 \\
\hline $\mathrm{C}$ & 0.9329705503 & 2.9566373615 & 0.2769960083 \\
\hline $\mathrm{C}$ & -1.1228848952 & 3.1784296731 & -0.9583809663 \\
\hline $\mathrm{C}$ & -3.4804529026 & 3.0865486107 & -1.4021089054 \\
\hline $\mathrm{C}$ & -5.2389516661 & 2.1246601112 & 0.4842936930 \\
\hline $\mathrm{C}$ & -5.3803744565 & -0.2367950880 & 1.1066803240 \\
\hline $\mathrm{C}$ & -4.1804652045 & -2.3702599985 & 1.3185202031 \\
\hline $\mathrm{C}$ & -2.1698515704 & -3.7861572794 & 1.1360214501 \\
\hline $\mathrm{C}$ & 0.1962397760 & -4.3520585314 & 0.7412814877 \\
\hline $\mathrm{C}$ & 2.3236133699 & -3.7964531559 & -1.0778559452 \\
\hline $\mathrm{C}$ & 4.6654418939 & -2.7437104116 & 0.1475367265 \\
\hline $\mathrm{C}$ & 5.7927293568 & -0.6101786875 & 0.4106996736 \\
\hline $\mathrm{C}$ & 5.2210498558 & 2.0926345979 & -0.3496120138 \\
\hline $\mathrm{C}$ & 3.5047000405 & 3.7449494177 & 0.1503821400 \\
\hline $\mathrm{C}$ & 1.1488346048 & 4.4049281499 & 0.0542367502 \\
\hline $\mathrm{C}$ & -1.2849086961 & 4.4740866778 & -0.2797569893 \\
\hline $\mathrm{C}$ & -3.6394981086 & 3.8455874754 & -0.1670684268 \\
\hline $\mathrm{C}$ & -5.3810418804 & 1.7146602320 & -0.9035472120 \\
\hline $\mathrm{C}$ & -5.7214733256 & -0.5854870886 & -0.2674258441 \\
\hline $\mathrm{C}$ & -4.5220080867 & -2.6925470827 & -0.0696042374 \\
\hline $\mathrm{C}$ & -2.4435466305 & -3.9711613760 & -0.2944457938 \\
\hline $\mathrm{C}$ & -0.0458972902 & -4.3241118839 & -0.7021197852 \\
\hline $\mathrm{C}$ & 2.5945932480 & -4.0244657785 & 0.3364074223 \\
\hline $\mathrm{C}$ & 1.0381908248 & -4.1155322971 & -1.5698959908 \\
\hline $\mathrm{C}$ & 3.2612701039 & -3.0669687857 & -1.8417999130 \\
\hline $\mathrm{H}$ & 3.0191102150 & -2.8362880935 & -2.8773039146 \\
\hline $\mathrm{C}$ & 4.9075939719 & -1.2194390268 & -1.7911140162 \\
\hline $\mathrm{H}$ & 4.6407476024 & -0.9551413648 & -2.8124809774 \\
\hline $\mathrm{C}$ & 5.4690032039 & 1.1489213052 & -1.3258596350 \\
\hline $\mathrm{H}$ & 5.3566971182 & 1.4168144742 & -2.3744945687 \\
\hline $\mathrm{C}$ & 4.5955435458 & 3.3907698847 & -0.6137263900 \\
\hline $\mathrm{H}$ & 4.8392839978 & 3.9285520300 & -1.5268922773 \\
\hline $\mathrm{C}$ & -0.0435720409 & 5.1656856794 & -0.0397630213 \\
\hline $\mathrm{H}$ & -0.0270402176 & 6.2465788458 & 0.0745567422 \\
\hline $\mathrm{C}$ & -2.5757656040 & 4.7822858637 & 0.1374580116 \\
\hline $\mathrm{H}$ & -2.7552875532 & 5.6067866212 & 0.8230867846 \\
\hline $\mathrm{C}$ & -4.5870462252 & 3.3754049933 & 0.7434174134 \\
\hline $\mathrm{H}$ & -4.6086724552 & 3.7871286678 & 1.7501012184 \\
\hline $\mathrm{C}$ & -5.3782344022 & 1.1309827757 & 1.4749293249 \\
\hline $\mathrm{H}$ & -5.1641460862 & 1.3924111077 & 2.5092881264 \\
\hline $\mathrm{C}$ & -4.7604143804 & -1.2282016356 & 1.9081569309 \\
\hline $\mathrm{H}$ & -4.4881300994 & -0.9754485456 & 2.9309125263 \\
\hline $\mathrm{C}$ & -3.1118787090 & -3.0738286630 & 1.9173653336 \\
\hline $\mathrm{H}$ & -2.8746206458 & -2.8779044239 & 2.9609729943 \\
\hline $\mathrm{C}$ & -0.9062079174 & -4.1474987729 & 1.6185458696 \\
\hline $\mathrm{H}$ & -0.7060056212 & -4.0682942621 & 2.6851092207 \\
\hline $\mathrm{C}$ & 1.5139946158 & -4.3696635138 & 1.1931474405 \\
\hline
\end{tabular}




\begin{tabular}{lccc}
$\mathrm{H}$ & 1.7058133253 & -4.4335162432 & 2.2622434386 \\
$\mathrm{C}$ & -1.3828076395 & -4.3081799631 & -1.1542157074 \\
$\mathrm{C}$ & -3.6961439293 & -3.5861349534 & -0.7926736941 \\
$\mathrm{C}$ & -5.4455104320 & -1.8950117261 & -0.7426710091 \\
$\mathrm{C}$ & -5.9013586743 & 0.4332142067 & -1.2028110532 \\
$\mathrm{C}$ & -4.5489718376 & 2.3091717818 & -1.8670156676 \\
$\mathrm{C}$ & -2.1567093912 & 2.7330232626 & -1.7638700477 \\
$\mathrm{C}$ & -0.0646037215 & 2.3666579393 & -0.4630778487 \\
$\mathrm{C}$ & 1.8368942783 & 2.3247728691 & 1.1746922246 \\
$\mathrm{C}$ & 4.1858799859 & 2.0816159309 & 1.8667281752 \\
$\mathrm{C}$ & 5.8561010662 & 0.4140626806 & 1.3754436660 \\
$\mathrm{C}$ & 5.5632929653 & -1.9261303832 & 0.8551145133 \\
$\mathrm{C}$ & 3.8376551056 & -3.6474651696 & 0.8506151753 \\
$\mathrm{C}$ & 2.4959492402 & 4.7648153677 & -0.1056461349 \\
$\mathrm{H}$ & 2.7757614662 & 5.7312915281 & -0.5167412553 \\
$\mathrm{H}$ & -2.0109053675 & 1.8800799275 & -2.4221313862 \\
$\mathrm{H}$ & -4.4718733395 & 1.8358639874 & -2.8430733366 \\
$\mathrm{H}$ & -6.0416353202 & 0.1630941872 & -2.2470159930 \\
$\mathrm{H}$ & -5.6825774725 & -2.1266503042 & -1.7788628097 \\
$\mathrm{H}$ & -3.9189806827 & -3.7590558069 & -1.8435042777 \\
$\mathrm{H}$ & -1.5749408492 & -4.3579722847 & -2.2240391468 \\
$\mathrm{H}$ & 0.8416206804 & -4.0084416908 & -2.6348272465 \\
$\mathrm{H}$ & 4.0474913051 & -3.8271025828 & 1.9029154660 \\
$\mathrm{H}$ & 5.7686418239 & -2.1627693762 & 1.8968174412 \\
$\mathrm{H}$ & 5.9231925392 & 0.1307218243 & 2.4233739962 \\
$\mathrm{H}$ & 3.9911314280 & 1.5722299254 & 2.8069819951 \\
$\mathrm{H}$ & 1.5503294517 & 1.4166350911 & 1.6982370084 \\
$\mathrm{H}$ & -0.1312512621 & 1.2859699289 & -0.5580458293 \\
\hline & & &
\end{tabular}


Table S34. Cartesian Coordinates in Angstrom for the optimized structure of Möbius-[13]cyclacene containing one Dewar benzenoid.

\begin{tabular}{|c|c|c|c|}
\hline Atom & $\mathrm{x}$-coordinate & y-coordinate & z-coordinate \\
\hline $\mathrm{C}$ & 2.3696235965 & -3.5669214705 & 0.2533917802 \\
\hline $\mathrm{C}$ & -0.0319110786 & -3.9371076790 & 0.5801441865 \\
\hline $\mathrm{C}$ & -2.6689638248 & -3.6003023007 & -0.4508549526 \\
\hline $\mathrm{C}$ & -4.8324186013 & -2.5023227022 & -0.1277061410 \\
\hline $\mathrm{C}$ & -6.0784132273 & -0.4208909109 & -0.2084865263 \\
\hline $\mathrm{C}$ & -5.5850208681 & 1.8504207026 & -0.8147929281 \\
\hline $\mathrm{C}$ & -3.5099051520 & 2.9431400567 & -1.3002972816 \\
\hline $\mathrm{C}$ & -1.1439887835 & 4.0673220215 & -0.2196593039 \\
\hline $\mathrm{C}$ & 1.2742884734 & 3.7749471823 & 0.0703409468 \\
\hline $\mathrm{C}$ & 3.6434786633 & 3.1744707096 & 0.2283784155 \\
\hline $\mathrm{C}$ & 4.5718214234 & -2.5009894437 & 0.1816913932 \\
\hline $\mathrm{C}$ & 2.1311420778 & -3.1735731397 & -1.1249917181 \\
\hline $\mathrm{C}$ & -0.2476046052 & -3.7489563647 & -0.8515777820 \\
\hline $\mathrm{C}$ & -2.4230999937 & -3.5420521236 & 0.9884779749 \\
\hline $\mathrm{C}$ & -4.4951887174 & -2.2547039488 & 1.2713476358 \\
\hline $\mathrm{C}$ & -5.6870351844 & -0.1188229715 & 1.1648855178 \\
\hline $\mathrm{C}$ & -5.3702565070 & 2.2294468346 & 0.5809017484 \\
\hline $\mathrm{C}$ & -3.5673551567 & 3.7379398071 & -0.0708185223 \\
\hline $\mathrm{C}$ & -1.1392515722 & 2.7654230080 & -0.8895799984 \\
\hline $\mathrm{C}$ & 1.0086314945 & 2.3457612990 & 0.1460861298 \\
\hline $\mathrm{C}$ & 3.2541356449 & 1.9943953017 & 0.9942995835 \\
\hline $\mathrm{C}$ & 4.2934238857 & -1.9825260266 & -1.1556027304 \\
\hline $\mathrm{C}$ & 5.6370593428 & -1.9423668389 & 0.9594174629 \\
\hline $\mathrm{C}$ & 3.6388545706 & -3.3296155630 & 0.8087256462 \\
\hline $\mathrm{H}$ & 3.8292022883 & -3.6479000383 & 1.8315836109 \\
\hline $\mathrm{C}$ & 1.2851058698 & -3.9800400542 & 1.0550687710 \\
\hline $\mathrm{H}$ & 1.4611567244 & -4.1741846130 & 2.1111170381 \\
\hline $\mathrm{C}$ & -1.1432880346 & -3.8756901814 & 1.4556489060 \\
\hline $\mathrm{H}$ & -0.9604957500 & -3.9030724012 & 2.5278919709 \\
\hline $\mathrm{C}$ & -3.4005349175 & -2.9432816136 & 1.8225895268 \\
\hline $\mathrm{H}$ & -3.1681736040 & -2.8093596083 & 2.8770136189 \\
\hline $\mathrm{C}$ & -5.5939452241 & 1.2516223292 & 1.5602860252 \\
\hline $\mathrm{H}$ & -5.3494237434 & 1.4773074348 & 2.5961085812 \\
\hline $\mathrm{C}$ & -4.5829198697 & 3.4032524301 & 0.8384660287 \\
\hline $\mathrm{H}$ & -4.5576193930 & 3.8190454809 & 1.8432219515 \\
\hline $\mathrm{C}$ & -2.4069316678 & 4.5197361405 & 0.2289936554 \\
\hline $\mathrm{H}$ & -2.4624902522 & 5.3210194395 & 0.9620567319 \\
\hline $\mathrm{C}$ & 0.1324453335 & 4.6212955691 & 0.0471218301 \\
\hline $\mathrm{H}$ & 0.2345692501 & 5.6753566432 & 0.2944260437 \\
\hline $\mathrm{C}$ & 2.6395930603 & 4.1360842921 & -0.0114823139 \\
\hline $\mathrm{H}$ & 2.9127715271 & 5.1272024900 & -0.3667005720 \\
\hline $\mathrm{C}$ & 4.9284366563 & 3.1273627152 & -0.4266200800 \\
\hline $\mathrm{C}$ & 5.0861783998 & -0.8871794836 & -1.6854911372 \\
\hline $\mathrm{H}$ & 4.8218774328 & -0.4786850343 & -2.6585359413 \\
\hline $\mathrm{C}$ & 4.2662897110 & 1.2047249039 & 1.6321078143 \\
\hline $\mathrm{C}$ & 1.9384073651 & 1.5491179130 & 0.8278159511 \\
\hline $\mathrm{C}$ & -0.1295235662 & 1.8769248490 & -0.5469463299 \\
\hline $\mathrm{C}$ & -2.2748670573 & 2.4385742456 & -1.6756988704 \\
\hline $\mathrm{C}$ & -4.7101932026 & 2.3485526751 & -1.7808391231 \\
\hline $\mathrm{C}$ & -6.2617307682 & 0.6396009322 & -1.1128925728 \\
\hline
\end{tabular}




\begin{tabular}{cccc}
$\mathrm{C}$ & -5.8196118651 & -1.7013314874 & -0.7390171172 \\
$\mathrm{C}$ & -3.9597479820 & -3.2647872684 & -0.9214265233 \\
$\mathrm{C}$ & -1.5833917823 & -3.7535407584 & -1.3250559022 \\
$\mathrm{C}$ & 0.8447249967 & -3.4080672457 & -1.6665886633 \\
$\mathrm{C}$ & 3.1324399972 & -2.4357930857 & -1.8019614994 \\
$\mathrm{C}$ & -5.0808751070 & -1.1353009058 & 1.9226497549 \\
$\mathrm{H}$ & -4.7864948733 & -0.9227949175 & 2.9485083136 \\
$\mathrm{H}$ & -4.7253260613 & 1.9189964882 & -2.7795598838 \\
$\mathrm{H}$ & -2.2145316750 & 1.6356849503 & -2.4060121168 \\
$\mathrm{H}$ & -0.2166144759 & 0.8217718782 & -0.7946661124 \\
$\mathrm{H}$ & 1.6716439002 & 0.5387377645 & 1.1287114276 \\
$\mathrm{H}$ & 5.7814995597 & -2.2778896865 & 1.9839466612 \\
$\mathrm{H}$ & 2.9210788575 & -2.0842084421 & -2.8100737091 \\
$\mathrm{H}$ & 0.6660227299 & -3.1820583442 & -2.7159614418 \\
$\mathrm{H}$ & -1.7608669826 & -3.6950805498 & -2.3968859989 \\
$\mathrm{H}$ & -4.1713330387 & -3.3623869318 & -1.9841033760 \\
$\mathrm{H}$ & -6.0710772046 & -1.8905417592 & -1.7802461192 \\
$\mathrm{H}$ & -6.4601716177 & 0.4036521309 & -2.1558354249 \\
$\mathrm{C}$ & 5.7567722129 & 2.0969287767 & -0.0561841999 \\
$\mathrm{C}$ & 5.5331724424 & 1.3567795123 & 1.1461103141 \\
$\mathrm{C}$ & 6.7614033975 & 1.0457237576 & -0.5363049764 \\
$\mathrm{C}$ & 6.7798328267 & 0.4702493537 & 0.9603082443 \\
$\mathrm{H}$ & 7.6643455170 & 1.2874525791 & -1.1030037345 \\
$\mathrm{H}$ & 7.6318813719 & 0.5863839701 & 1.6331323963 \\
$\mathrm{C}$ & 6.0157148957 & -0.3150288110 & -0.8584037884 \\
$\mathrm{C}$ & 6.2474843375 & -0.8391298687 & 0.4528062243 \\
$\mathrm{H}$ & 5.1200166749 & 3.7899694882 & -1.2672911733 \\
$\mathrm{H}$ & 3.9853412399 & 0.4124901117 & 2.3217384359 \\
\hline & & &
\end{tabular}


Table S35. Cartesian Coordinates in Angstrom for the optimized structure of Möbius-[14]cyclacene containing no Dewar benzenoids.

\begin{tabular}{|c|c|c|c|}
\hline Atom & $\mathrm{x}$-coordinate & y-coordinate & z-coordinate \\
\hline $\mathrm{C}$ & -2.7003388348 & -4.0055088742 & 1.1571455326 \\
\hline $\mathrm{C}$ & -4.7102611963 & -2.5895795553 & 1.2843978849 \\
\hline $\mathrm{C}$ & -6.2098915164 & -0.8235231131 & -0.3557242180 \\
\hline $\mathrm{C}$ & -5.8487779447 & 1.4789412615 & -0.9865640238 \\
\hline $\mathrm{C}$ & -3.9651012281 & 2.9201523526 & -1.3643026535 \\
\hline $\mathrm{C}$ & -1.6458455971 & 3.2431379546 & -0.8029603656 \\
\hline $\mathrm{C}$ & 0.4342717427 & 3.3131445146 & 0.4235673545 \\
\hline $\mathrm{C}$ & 2.8927673842 & 4.5065266935 & 0.3681401737 \\
\hline $\mathrm{C}$ & 4.9833448037 & 3.3256966920 & -0.0739414550 \\
\hline $\mathrm{C}$ & 5.9507894581 & 1.1997799755 & -0.7952387158 \\
\hline $\mathrm{C}$ & 5.4947410189 & -1.1598486002 & -1.2679673827 \\
\hline $\mathrm{C}$ & 3.9546450910 & -3.0693018274 & -1.3172232941 \\
\hline $\mathrm{C}$ & 2.0780355977 & -4.3973588069 & 0.3778913157 \\
\hline $\mathrm{C}$ & -0.3375816381 & -4.6069517831 & 0.7985364310 \\
\hline $\mathrm{C}$ & -2.9702593797 & -4.2500295848 & -0.2590961727 \\
\hline $\mathrm{C}$ & -5.0326986391 & -2.9384949562 & -0.0953411351 \\
\hline $\mathrm{C}$ & -5.9153138391 & -0.4656053681 & 1.0263699493 \\
\hline $\mathrm{C}$ & -5.7979090454 & 1.9007458482 & 0.4093755592 \\
\hline $\mathrm{C}$ & -4.2575804851 & 3.7074000489 & -0.1650010894 \\
\hline $\mathrm{C}$ & -1.9584272570 & 4.5463727943 & -0.1980977061 \\
\hline $\mathrm{C}$ & 0.4595315605 & 4.7677735855 & 0.1944761590 \\
\hline $\mathrm{C}$ & 2.6577737744 & 3.4375150675 & 1.3518652498 \\
\hline $\mathrm{C}$ & 4.9647719557 & 2.7685646084 & 1.2813547171 \\
\hline $\mathrm{C}$ & 6.2320318663 & 0.8401141801 & 0.5886729346 \\
\hline $\mathrm{C}$ & 5.8606542243 & -1.5047846067 & 0.0947357280 \\
\hline $\mathrm{C}$ & 4.2757239842 & -3.3565479627 & 0.0733319456 \\
\hline $\mathrm{C}$ & 1.8164595373 & -4.2566087502 & -1.0537541482 \\
\hline $\mathrm{C}$ & -0.5774742605 & -4.6667387562 & -0.6446350174 \\
\hline $\mathrm{C}$ & 3.3520906168 & -4.0895733058 & 0.8598526496 \\
\hline $\mathrm{C}$ & 0.9866551686 & -4.6293899116 & 1.2551186492 \\
\hline $\mathrm{H}$ & 1.1768940775 & -4.6377034883 & 2.3262933001 \\
\hline $\mathrm{C}$ & -1.4299055676 & -4.3429784299 & 1.6599302147 \\
\hline $\mathrm{H}$ & -1.2330040136 & -4.2160505876 & 2.7225395225 \\
\hline $\mathrm{C}$ & -3.6406093271 & -3.2626631110 & 1.9089892224 \\
\hline $\mathrm{H}$ & -3.4073561133 & -3.0311813422 & 2.9463164407 \\
\hline $\mathrm{C}$ & -5.3058760759 & -1.4311869234 & 1.8476556870 \\
\hline $\mathrm{H}$ & -5.0534403081 & -1.1648755539 & 2.8720630446 \\
\hline $\mathrm{C}$ & -5.9577052505 & 0.9159970682 & 1.3909963366 \\
\hline $\mathrm{H}$ & -5.8110855301 & 1.1835038759 & 2.4355338658 \\
\hline $\mathrm{C}$ & -3.2990853448 & 4.7399149034 & 0.1572053991 \\
\hline $\mathrm{H}$ & -3.5813031673 & 5.5603317239 & 0.8127273031 \\
\hline $\mathrm{C}$ & -0.8207877493 & 5.3717889922 & 0.0671821399 \\
\hline $\mathrm{H}$ & -0.9386320853 & 6.4445999991 & 0.1994302538 \\
\hline $\mathrm{C}$ & 1.7410895889 & 5.3335148575 & 0.0829840248 \\
\hline $\mathrm{H}$ & 1.8708700012 & 6.3351818022 & -0.3197085571 \\
\hline $\mathrm{C}$ & 4.0937791163 & 4.4503739137 & -0.3241667039 \\
\hline $\mathrm{H}$ & 4.2630215271 & 5.0904619333 & -1.1869225867 \\
\hline $\mathrm{C}$ & 5.5739796659 & 2.5683026926 & -1.0723286101 \\
\hline $\mathrm{H}$ & 5.4956291607 & 2.8894262040 & -2.1088577031 \\
\hline $\mathrm{C}$ & 5.7269285947 & 0.1748625929 & -1.7153665826 \\
\hline
\end{tabular}




\begin{tabular}{cccc}
$\mathrm{H}$ & 5.4558383973 & 0.4324171957 & -2.7373425690 \\
$\mathrm{C}$ & 4.6596095615 & -2.0470624607 & -1.9802693828 \\
$\mathrm{H}$ & 4.3809569683 & -1.7989658955 & -3.0024389287 \\
$\mathrm{C}$ & 2.7896310731 & -3.6699615183 & -1.8695715666 \\
$\mathrm{H}$ & 2.5644733905 & -3.5051752035 & -2.9212649845 \\
$\mathrm{C}$ & 5.3370688830 & -2.6724504449 & 0.6797363674 \\
$\mathrm{C}$ & 6.3949724699 & -0.5027768455 & 0.9366580747 \\
$\mathrm{C}$ & 5.8580199532 & 1.7473664015 & 1.6171799958 \\
$\mathrm{C}$ & 3.7637086141 & 2.8903843835 & 2.0159006914 \\
$\mathrm{C}$ & 1.4179972280 & 2.7867476626 & 1.2685336448 \\
$\mathrm{C}$ & -0.5166966170 & 2.5871358930 & -0.3047834240 \\
$\mathrm{C}$ & -2.6174023269 & 2.6686373185 & -1.6399942273 \\
$\mathrm{C}$ & -4.9775322496 & 2.0932257032 & -1.8965611770 \\
$\mathrm{C}$ & -6.3544690212 & 0.1985589655 & -1.3067910508 \\
$\mathrm{C}$ & -5.9371446457 & -2.1331461830 & -0.8067683189 \\
$\mathrm{C}$ & -4.2159648010 & -3.8574904131 & -0.7842074062 \\
$\mathrm{C}$ & -1.9167850795 & -4.6484226211 & -1.0995718961 \\
$\mathrm{C}$ & 0.5075707117 & -4.5549081296 & -1.5226236241 \\
$\mathrm{C}$ & -5.2339127527 & 3.1981327283 & 0.6902853160 \\
$\mathrm{H}$ & -5.3645999633 & 3.6317141853 & 1.6792754363 \\
$\mathrm{H}$ & -0.4014229646 & 1.5151726663 & -0.4436438128 \\
$\mathrm{H}$ & 1.2964612724 & 1.7984670712 & 1.7045099051 \\
$\mathrm{H}$ & 3.6109261111 & 2.2440523531 & 2.8767555053 \\
$\mathrm{H}$ & 5.8894463572 & 1.3930192295 & 2.6448477157 \\
$\mathrm{H}$ & 6.5867597626 & -0.7539029304 & 1.9774668974 \\
$\mathrm{H}$ & 5.5673892995 & -2.8805136261 & 1.7224247172 \\
$\mathrm{H}$ & 3.5634329374 & -4.2192363727 & 1.9191948303 \\
$\mathrm{H}$ & 0.3161900223 & -4.5231720142 & -2.5932121412 \\
$\mathrm{H}$ & -2.1086410747 & -4.7528236582 & -2.1654095936 \\
$\mathrm{H}$ & -4.4294190678 & -4.0588228740 & -1.8317995412 \\
$\mathrm{H}$ & -6.1471783151 & -2.3779777359 & -1.8457432660 \\
$\mathrm{H}$ & -6.4461811147 & -0.0770833911 & -2.3549374881 \\
$\mathrm{H}$ & -4.8317633841 & 1.6352878880 & -2.8719097572 \\
$\mathrm{H}$ & -2.3515215787 & 1.8517087194 & -2.3061595529 \\
\hline & & &
\end{tabular}


Table S36. Cartesian Coordinates in Angstrom for the optimized structure of Möbius-[14]cyclacene containing one Dewar benzenoid.

\begin{tabular}{|c|c|c|c|}
\hline Atom & $\mathrm{x}$-coordinate & $y$-coordinate & $\mathrm{z}$-coordinate \\
\hline $\mathrm{C}$ & 4.1664298524 & 3.2875882051 & 0.2703852598 \\
\hline $\mathrm{C}$ & 1.5031376839 & 2.5150861509 & 0.3473432114 \\
\hline $\mathrm{C}$ & -0.6566165247 & 2.9099583417 & -0.6852378338 \\
\hline $\mathrm{C}$ & -2.9700631047 & 3.2429603655 & -1.3193076686 \\
\hline $\mathrm{C}$ & -5.2958171459 & 2.6643287973 & -1.0962085501 \\
\hline $\mathrm{C}$ & -6.5567552309 & 0.7287756153 & -0.4290575777 \\
\hline $\mathrm{C}$ & -5.7020241952 & -1.3340802051 & 1.3045727207 \\
\hline $\mathrm{C}$ & -4.0269800384 & -3.1249006989 & 1.2092644061 \\
\hline $\mathrm{C}$ & -1.7798121335 & -4.0605832644 & 0.8586147851 \\
\hline $\mathrm{C}$ & 0.6434797048 & -4.1981141703 & 0.4645590140 \\
\hline $\mathrm{C}$ & 3.0157072585 & -3.6442096166 & 0.1877746391 \\
\hline $\mathrm{C}$ & 4.8767124181 & -1.9414401453 & -1.1665531916 \\
\hline $\mathrm{C}$ & 3.7858055630 & 2.1432705628 & 1.0915740869 \\
\hline $\mathrm{C}$ & 1.8036660681 & 3.9246050534 & 0.1520507631 \\
\hline $\mathrm{C}$ & -0.6014541874 & 4.2828378421 & -0.1819080147 \\
\hline $\mathrm{C}$ & -3.0556119990 & 4.2031885944 & -0.2157862489 \\
\hline $\mathrm{C}$ & -5.2042588036 & 3.1464374984 & 0.2816285077 \\
\hline $\mathrm{C}$ & -6.2200752332 & 1.0316519352 & 0.9584281235 \\
\hline $\mathrm{C}$ & -6.0751403013 & -1.6184186402 & -0.0767843115 \\
\hline $\mathrm{C}$ & -4.3169301543 & -3.3064943472 & -0.2105663834 \\
\hline $\mathrm{C}$ & -2.0048696287 & -4.0399756401 & -0.5856610460 \\
\hline $\mathrm{C}$ & 0.4274295956 & -3.9575512639 & -0.9591975074 \\
\hline $\mathrm{C}$ & 2.7710778165 & -3.2308236774 & -1.1832606864 \\
\hline $\mathrm{C}$ & 5.1571053960 & -2.4583864867 & 0.1700719587 \\
\hline $\mathrm{C}$ & 3.7436949000 & -2.4332784648 & -1.8336030151 \\
\hline $\mathrm{C}$ & 5.6407777842 & -0.8195421824 & -1.6821915271 \\
\hline $\mathrm{H}$ & 5.3791767503 & -0.4169685114 & -2.6583933690 \\
\hline $\mathrm{C}$ & 5.4349728537 & 3.2132221917 & -0.4144819239 \\
\hline $\mathrm{H}$ & 5.6128358766 & 3.8571377605 & -1.2725594599 \\
\hline $\mathrm{C}$ & 3.1713543497 & 4.2515939320 & 0.0127525941 \\
\hline $\mathrm{H}$ & 3.4506128086 & 5.2152344334 & -0.4076677661 \\
\hline $\mathrm{C}$ & 0.6894089198 & 4.8027068216 & 0.0559301373 \\
\hline $\mathrm{H}$ & 0.8311980917 & 5.8719483577 & 0.1957752894 \\
\hline $\mathrm{C}$ & -4.2280133097 & 4.1600752973 & 0.5593670408 \\
\hline $\mathrm{H}$ & -4.2626738057 & 4.6943959680 & 1.5060118122 \\
\hline $\mathrm{C}$ & -5.8004387753 & 2.3617035928 & 1.2787546926 \\
\hline $\mathrm{H}$ & -5.6427165028 & 2.6234169324 & 2.3228792472 \\
\hline $\mathrm{C}$ & -5.9768998938 & -0.0419496797 & 1.8310176036 \\
\hline $\mathrm{H}$ & -5.6865526028 & 0.1695265204 & 2.8581099343 \\
\hline $\mathrm{C}$ & -4.8229825382 & -2.2206813505 & 1.9545394452 \\
\hline $\mathrm{H}$ & -4.5584798488 & -2.0329249247 & 2.9931379968 \\
\hline $\mathrm{C}$ & -2.8325605610 & -3.6655516243 & 1.7184180941 \\
\hline $\mathrm{H}$ & -2.6305824065 & -3.5898304168 & 2.7849111693 \\
\hline $\mathrm{C}$ & -0.4766674081 & -4.2872709281 & 1.3247140846 \\
\hline $\mathrm{H}$ & -0.3034729105 & -4.3670676685 & 2.3959056485 \\
\hline $\mathrm{C}$ & 1.9531362174 & -4.1592104527 & 0.9581607547 \\
\hline $\mathrm{H}$ & 2.1298203526 & -4.3825787641 & 2.0082654317 \\
\hline $\mathrm{C}$ & 4.2585100469 & -3.3424052248 & 0.7699030699 \\
\hline $\mathrm{H}$ & 4.4507894556 & -3.6688660871 & 1.7897685775 \\
\hline $\mathrm{C}$ & 1.5048159107 & -3.5178694112 & -1.7468569629 \\
\hline
\end{tabular}




\begin{tabular}{lccc}
$\mathrm{C}$ & -0.8999034685 & -4.0338416054 & -1.4499657189 \\
$\mathrm{C}$ & -3.3234405760 & -3.8335509135 & -1.0519420215 \\
$\mathrm{C}$ & -5.4721208454 & -2.7006656094 & -0.7457938166 \\
$\mathrm{C}$ & -6.7164930451 & -0.6166696091 & -0.8288575928 \\
$\mathrm{C}$ & -6.2597881126 & 1.6754730598 & -1.4206798232 \\
$\mathrm{C}$ & -4.1793527297 & 2.8003964153 & -1.9176960942 \\
$\mathrm{C}$ & -1.7739309845 & 2.5615402402 & -1.4852713936 \\
$\mathrm{C}$ & 0.3242809767 & 2.0245581941 & -0.2549036656 \\
$\mathrm{C}$ & 2.4519149055 & 1.7312654525 & 1.0217885287 \\
$\mathrm{C}$ & 4.8073873616 & 1.3425830353 & 1.7046571466 \\
$\mathrm{C}$ & 6.1879116020 & -1.8638677266 & 0.9683916886 \\
$\mathrm{C}$ & -1.8497110076 & 4.8845830112 & 0.1178102389 \\
$\mathrm{H}$ & -1.8797025189 & 5.7755466256 & 0.7406772875 \\
$\mathrm{H}$ & -6.3860841012 & 1.3952011572 & -2.4638449059 \\
$\mathrm{H}$ & -6.9567956897 & -0.8221527125 & -1.8696183807 \\
$\mathrm{H}$ & -5.7059080934 & -2.8585733210 & -1.7964231121 \\
$\mathrm{H}$ & -3.5147000394 & -3.8804687985 & -2.1219140981 \\
$\mathrm{H}$ & -1.0706798697 & -3.9293751529 & -2.5194575751 \\
$\mathrm{H}$ & 1.3241714590 & -3.2663552512 & -2.7900929312 \\
$\mathrm{H}$ & 3.5301792925 & -2.0763907122 & -2.8394370957 \\
$\mathrm{H}$ & 6.3317430598 & -2.2022905576 & 1.9920052211 \\
$\mathrm{H}$ & 4.5368466030 & 0.5763391971 & 2.4272708353 \\
$\mathrm{H}$ & 2.1777481097 & 0.7450643941 & 1.3896082093 \\
$\mathrm{H}$ & 0.1968724797 & 0.9543198900 & -0.3982360856 \\
$\mathrm{H}$ & -1.7362594357 & 1.6716956401 & -2.1085520129 \\
$\mathrm{H}$ & -4.1419539213 & 2.2584014736 & -2.8594232967 \\
$\mathrm{C}$ & 6.7737596228 & -0.7437519491 & 0.4716369912 \\
$\mathrm{C}$ & 6.5434986023 & -0.2261006595 & -0.8420723424 \\
$\mathrm{C}$ & 7.2988301653 & 0.5705809368 & 0.9770864293 \\
$\mathrm{C}$ & 7.2726480239 & 1.1397294531 & -0.5225435225 \\
$\mathrm{H}$ & 8.1559607059 & 0.6869851301 & 1.6435009684 \\
$\mathrm{H}$ & 8.1725357286 & 1.3899251116 & -1.0903277671 \\
$\mathrm{C}$ & 6.0571621987 & 1.4605824834 & 1.1720771683 \\
$\mathrm{C}$ & 6.2624663131 & 2.1851527292 & -0.0428190092 \\
\hline & & &
\end{tabular}

